Supporting Information

\title{
Target and nontarget screening of PFAS in biosolids, composts, and other organic waste products for land application in France
}

Gabriel Munoz, ${ }^{1}$ Aurélia M. Michaud, ${ }^{2,3}$ Min Liu, ${ }^{4}$ Sung Vo Duy, ${ }^{1}$ Denis Montenach, ${ }^{5}$ Camille Resseguier, ${ }^{2}$ Françoise Watteau, ${ }^{6}$ Valérie Sappin-Didier, ${ }^{7}$ Frédéric Feder, ${ }^{8,9}$ Thierry Morvan, ${ }^{3}$ Sabine Houot, ${ }^{2}$ Mélanie Desrosiers, ${ }^{10}$ Jinxia Liu, ${ }^{4}$ Sébastien Sauvé ${ }^{1, *}$

${ }^{1}$ Département de Chimie, Université de Montréal, Montréal, QC H2V 0B3, Canada.

2 INRAE, UMR ECOSYS, Ecologie fonctionnelle et écotoxicologie des agroécosystèmes, AgroParisTech, Université Paris-Saclay, 78850 Thiverval-Grignon, France.

${ }^{3}$ INRAE, UMR SAS, Sol Agro et hydrosystème Spatialisation, 35000 Rennes, France.

${ }^{4}$ Department of Civil Engineering, McGill University, Montreal, QC H3A 0C3, Canada.

${ }^{5}$ INRAE, UE UEAV, Unité d'expérimentation agronomique et viticole, 68000 Colmar, France.

${ }^{6}$ INRAE, Laboratoire Sols et Environnement, Université de Lorraine, 54000 Nancy, France.

7 INRAE, UMR ISPA, Interactions Sol Plante Atmosphère, Bordeaux Sciences Agro, 33140 Villenave d'Ornon, France.

${ }^{8}$ CIRAD, UPR Recyclage et risque, 97408 Saint-Denis, Réunion, France.

${ }^{9}$ CIRAD, UPR Recyclage et risque, Université de Montpellier, 34398 Montpellier, France.

${ }^{10}$ Centre d'expertise en analyse environnementale du Québec, ministère de l'Environnement et de la Lutte contre les changements climatiques, Québec, QC G1P 3W8, Canada.

${ }^{*}$ Corresponding author.

sebastien.sauve@umontreal.ca 


\section{Table of Contents}

\section{List of abbreviations and acronyms}

\section{SI Texts}

Text S1. Details on chemicals and materials.

Text S2. Targeted PFAS analysis.

Text S3. Occurrence of perfluoroalkyl acids in organic waste products.

\section{SI Tables}

Table S1. Surrogate internal standards used in this study, including acronyms, names, $\mathrm{m} / \mathrm{z}$, ion mode, and supplier company.

Table S2. Acronym, name, and supplier of the 46 target PFAS with certified standards.

Table S3. Samples of organic waste products (OWP) per origin (urban, livestock manure, industry), with number of replicates per OWP sample, sites, and year of collection.

Table S4. Samples of organic waste products (OWP), with the following metadata presented: Organic waste product name and code, origin, year of production, experimental site where it is studied (La Bouzule - Grand-Est, Couhins - Nouvelle-Aquitaine, EFELE - Bretagne, PROspective - Grand-Est, QualiAgro - Ile-de-France, La Réunion - French Overseas), treatment process, raw materials, and number of replicates par sample.

Table S5. Chemical properties of organic waste products (OWP) per site and year, with the following data presented: dry matter (DM; \% of fresh matter - FM), $\mathrm{pH}$, content of carbonates $\left(\mathrm{CaCO}_{3}\right)$, organic carbon $(\mathrm{OC})$, nitrogen $(\mathrm{N})$, phosphorus $\left(\mathrm{P}_{2} \mathrm{O}_{5}\right)$ and potassium $(\mathrm{K})$ (g per kilogram of dry matter).

Table S6. Average chemical properties of the organic waste products (OWP), with the following data presented: dry matter (DM; \% of fresh matter - $\mathrm{FM}), \mathrm{pH}$, content of carbonates $\left(\mathrm{CaCO}_{3}\right)$, organic carbon $(\mathrm{OC})$, nitrogen $(\mathrm{N})$, phosphorus $\left(\mathrm{P}_{2} \mathrm{O}_{5}\right)$ and potassium $(\mathrm{K})$ (g per kilogram of dry matter).

Table S7. Details on the UHPLC-HRMS instrumental method for PFAS analysis.

Table S8. Instrumental performance, including method limits of detection $(\mu \mathrm{g} / \mathrm{kg}), R^{2}$, accuracy\% $(n=4)$ and corresponding intraday precision $(R S D \%, n=4)$, and interday precision $(\mathrm{RSD} \%, \mathrm{n}=12)$.

Table S9. Accuracy\% of continued calibration verification (CCV) standards run along the LCMS batch sequence $(n=6)$.

Table S10. Matrix spike recovery $\%$ of target PFAS to 4 model organic solid waste products.

Table S11. PFAS concentrations $(\mu \mathrm{g} / \mathrm{kg}$ ) determined in NIST SRM 2781 (standard reference material of domestic sludge).

Table S12. Summary of \% PFAS recovered by a secondary extraction on already solventextracted organic waste products, tested for six products with high PFAS content $\left(\Sigma_{46}\right.$ PFAS $>200 \mu \mathrm{g} / \mathrm{kg}$ ).

Table S13. Details on the 160 PFAS detected in the present study, including class, homolog acronym, ion formula, ion mode, theoretical $\mathrm{m} / \mathrm{z}$, observed $\mathrm{m} / \mathrm{z}$, exact mass error $(\delta \mathrm{ppm})$, and reference calibrant for quantification or semi-quantification. 
Table S14. Overall detection frequencies and concentration ranges $(\mu \mathrm{g} / \mathrm{kg})$ of the 160 detected PFAS homologs in OWP samples ( $\mathrm{N}=47)$.

Table S15. PFAS concentrations $(\mu \mathrm{g} / \mathrm{kg})$ in the 47 OWP samples, for each of the seven PFAS superclasses.

Table S16. PFAS classes detected in agricultural OWP screened in the present study $(n=21)$, ranked by maximum observed summed concentration ( $\sum$ class). Corresponding superclass (and ESI detection mode), detection rate, and representative homologs are also included.

\section{SI Figures}

Figure S1. Principal component analysis (with Spearman correlation matrixes) of chemical properties for the studied organic waste products.

Figure S2. Chromatograms and elucidated high-resolution MS/MS spectra of PFAS detected at high-intensity level in biosolids. When available, a certified reference standard or AFFF material was also analyzed for confirmation.

- ESI(-) PFAS

(S2-a). PFOS.

(S2-b). H-PFOS.

(S2-c). PFOSi.

(S2-d). F5S-PFNS.

(S2-e). FHxSAA.

(S2-f). MeFNSAA.

(S2-g). EtFHxSAA.

(S2-h). EtFHpSAA.

(S2-i). EtFOSAA.

(S2-j). 6:2 FTSA-PrA (6:2 fluorotelomer sulfonamide).

(S2-k). 9:3 acid (9:3 FTCA).

(S2-I). 11:3 acid (11:3 FTCA).

(S2-m). 6:2 FTSO-PrAdDiMePrS (6:2 FTSAS-sulfoxide).

(S2-n). 6:2 FTSO2-PrAdDiMeEtS (6:2 FTSAS-sulfone).

(S2-0). 6:6 PhiA.

- ESI(+) PFAS

(S2-p). T-AmPr-FOSA (PFOSAmS).

(S2-q). AmPr-FOSA (PFOSAm).

(S2-r). CMeAmPr-FOSA (PFOSB).

(S2-s). 6:2 FTSA-PrB (6:2 FTAB).

(S2-t). 8:2 FTSA-PrB (8:2 FTAB). 
Figure S3. Principal component analysis biplot of active variables (seven PFAS superclasses) and individuals (OWP samples), performed on the global OWP dataset ( $N=47)$ (PCA type: Spearman). Individuals are color-coded according to the type of OWP (SLU: sewage sludge; C-GWS: compost of green wastes and sludge; C-BIOW: compost of biowastes; C-MSW: compost of municipal solid waste; DIG-UW: digestate of urban wastes; FYM-DC: farmyard manure of dairy cattle; PS: pig slurry; PM: poultry manure; C-FYM-DC: compost of farmyard manure of dairy cattle; DIG-PS: digestate of pig slurry; PSLU: paper sludge (mill sludge); ASH: combustion ashes).

Figure S4. Summed PFAS in a sample of composted residual municipal solid waste (C-MSW, QA site; Year: 2013), according to different monitoring scenarios. The left panel shows the graphs for scenarios 1,2 and the right panel for all three scenarios. (Scenario 1) summed target PFAAs (PFCAs + PFSAs); (Scenario 2) summed target PFAAs and commonly targeted ESI(-) precursors (FOSA, Me/EtFOSA, Me/EtFOSAA, X:2 FTSA); (Scenario 3) summed PFAS detected in the present study ( $\Sigma_{160}$ PFAS). The largest contribution was attributed to the fluorotelomer sulfonamidopropyl betaines (X:2 FTSA-PrB), especially the 6:2 homolog (6:2 FTSA-PrB also known as 6:2 FTAB). 


\section{List of abbreviations and acronyms used in the manuscript}

\begin{tabular}{|c|c|}
\hline $6: 2$ FTAB & 6:2 fluorotelomer sulfonamidopropyl betaine \\
\hline $6: 2$ FTSA & $6: 2$ fluorotelomer sulfonate \\
\hline 6:2 FTSA-PrB & 6:2 fluorotelomer sulfonamidopropyl betaine (formerly 6:2 FTAB) \\
\hline AFFFs & Aqueous film-forming foams \\
\hline AmPr-FASA & Dimethylammoniopropyl perfluoroalkyl sulfonamides \\
\hline AmPr-FHxSA & Dimethylammoniopropyl perfluorohexane sulfonamide (formerly PFHxSAm) \\
\hline AmPr-FOSA & Dimethylammoniopropyl perfluorooctane sulfonamide (formerly PFOSAm) \\
\hline ASH & Ashes \\
\hline C-BIOW & Compost of biowastes \\
\hline CCV & Continued Calibration Verification \\
\hline C-FYM-DC & Compost of farmyard manure of dairy cattle \\
\hline C-FYM-P & Compost of farmyard manure of pigs \\
\hline C-GWS & Compost of green wastes and sludge \\
\hline CMeAmPr-FASA & Perfluoroalkyl sulfonamidopropyl betaines \\
\hline CMeAmPr-FOSA & Perfluorooctane sulfonamidopropyl betaine (PFOSB) \\
\hline C-MSW & Compost of municipal solid wastes \\
\hline DIG-PS & Digestate of pig slurry \\
\hline DIG-UW & Digestate of urban wastes \\
\hline diPAPs & Polyfluoroalkyl phosphate diesters \\
\hline ECF & Electrochemical fluorination \\
\hline EPA & Environmental Protection Agency \\
\hline $\mathrm{ESI}(-)$ & Negative ion mode electrospray ionization \\
\hline $\mathrm{ESI}(+)$ & Positive ion mode electrospray ionization \\
\hline EtFOSAA & $\mathrm{N}$-ethyl perfluorooctane sulfonamido acetic acid \\
\hline EtOH-AmPr-FOSAPrS & Perfluorooctane sulfonamido N-propylsulfonate, N-propylammonio hydroxyethyl \\
\hline F5S-PFSA & Pentafluorosulfide perfluoroalkane sulfonates \\
\hline FASA & Perfluoroalkyl sulfonamides \\
\hline FASAA & Perfluoroalkyl sulfonamido acetic acids \\
\hline FOSA & Perfluorooctane sulfonamide \\
\hline FWHM & Full width at half maximum \\
\hline FYM-DC & Farmyard manure of dairy cattle \\
\hline HCA & Hierarchical cluster analysis \\
\hline HO-X:2 FTSA & Hydroxy- X:2 fluorotelomer sulfonates \\
\hline HRMS & High-resolution mass spectrometry \\
\hline ILIS & Isotope-labelled internal standard \\
\hline IUPAC & International Union of Pure and Applied Chemistry \\
\hline MeFOSAA & $\mathrm{N}$-methyl perfluorooctane sulfonamido acetic acid \\
\hline $\mathrm{MeOH}$ & Methanol \\
\hline Mono-PAPs & Polyfluoroalkyl phosphate esters \\
\hline NIST & National Institute of Standards and Technology \\
\hline OAmPr-FOAd & $\mathrm{N}$-oxide dimethylammoniopropyl perfluorooctaneamide (formerly PFOANO) \\
\hline OECD & The Organisation for Economic Co-operation and Development \\
\hline OWP & Organic Waste Products \\
\hline PCA & Principal Component Analysis \\
\hline PFAAs & Perfluoroalkyl acids \\
\hline PFAS & Per- and polyfluoroalkyl substances \\
\hline
\end{tabular}




\begin{tabular}{|c|c|}
\hline PFBA & Perfluorobutanoic acid \\
\hline PFCAs & Perfluoroalkyl carboxylates \\
\hline PFOA & Perfluorooctanoic acid \\
\hline PFOcDA & Perfluorooctadecanoic acid \\
\hline PFOS & Perfluorooctane sulfonate \\
\hline PFSAs & Perfluoroalkane sulfonates \\
\hline PFTeDA & Perfluorotetradecanoic acid \\
\hline PM & Poultry manure \\
\hline PS & Pig slurry \\
\hline PSLU & Paper sludge (mill sludge) \\
\hline RPLC & Reversed-phase liquid chromatography \\
\hline RSD & Relative standard deviation \\
\hline SI & Supporting Information \\
\hline SLU & Municipal sewage sludge \\
\hline SPAmPr-FHxSA & $\mathrm{N}$-sulfopropyl dimethylammoniopropyl perfluorohexane sulfonamide \\
\hline TAmPr-FASA & Trimethylammoniopropyl perfluoroalkyl sulfonamides \\
\hline TAmPr-FHxSA & Trimethylammoniopropyl perfluorohexane sulfonamide (formerly PFHxSAmS) \\
\hline TAmPr-FOSA & Trimethylammoniopropyl perfluorooctane sulfonamide (formerly PFOSAmS) \\
\hline UHPLC & Ultra-high-performance liquid chromatography \\
\hline U-PFSA & Unsaturated perfluoroalkane sulfonates \\
\hline $\mathrm{X}: 2$ FTCAs & $\mathrm{X}: 2$ fluorotelomer carboxylates \\
\hline $\mathrm{X}: 2$ FTSA-PrA & $\mathrm{X}: 2$ fluorotelomer sulfonamides \\
\hline $\mathrm{X}: 2$ FTSA-PrB & X:2 fluorotelomer sulfonamidopropyl betaines \\
\hline X:2 FTSA-PrDiMeAn & X:2 fluorotelomer sulfonamidopropyl dimethyl amines \\
\hline X:2 FTUCAs & $\mathrm{X}: 2$ fluorotelomer unsaturated carboxylates \\
\hline X:3 keto-FTTh-OH-PrAcid & $\mathrm{X}: 3$ ketone fluorotelomer thia hydroxy propanoic acids \\
\hline X:Y PhiA & $\mathrm{X}: \mathrm{Y}$ bisperfluoroalkyl phosphinic acids \\
\hline
\end{tabular}




\section{SI Texts}

Text S1. Details on chemicals and materials.

Analytical standards of perfluoroalkyl carboxylic acids (PFBA, PFPeA, PFHxA, PFHpA, PFOA, PFNA, PFDA, PFUnA, PFDoA, PFTrDA, PFTeDA), perfluoroalkane sulfonate potassium or sodium salts (PFPrS, PFBS, PFPeS, PFHxS, PFHpS, PFOS, PFDS), perfluoro-4ethylcyclohexane sulfonate potassium salt (PFECHS), perfluoroalkyl sulfonamides (FBSA, FHxSA, FOSA) and $\mathrm{N}$-alkyl derivatives (MeFOSA, EtFOSA), perfluorooctane sulfonamidoacetic acid (FOSAA) and $\mathrm{N}$-alkyl derivatives (MeFOSAA, EtFOSAA), n:2 fluorotelomer sulfonate sodium salts (4:2 FTSA, 6:2 FTSA, 8:2 FTSA), 6:2 perfluoroalkyl phosphate diester sodium salt (6:2 diPAP), and n:2 chlorinated polyfluoroalkyl ether sulfonate potassium salts (6:2 CI-PFESA and 8:2 Cl-PFESA) were purchased from Wellington Laboratories (Guelph, ON, Canada). The n:3 acids (3:3 acid, 5:3 acid, 7:3 acid) were obtained from DuPont USA (Wilmington, DE, USA) and Synquest Laboratories (Alachua, FL, USA). Perfluorohexane sulfonamidoalkyl amine (N-AP-FHxSA), perfluorohexane sulfonamidoalkyl ammonium (N-TAmP-FHxSA), and 6:2 fluorotelomer sulfonamidoalkyl betaine (6:2 FTSA-PrB) were obtained from Wellington Laboratories (Guelph, ON, Canada). Additional ESI(+) PFAS were custom synthesized at the Beijing FLUOBON Surfactant Institute (Beijing, China).

Surrogate internal standards including ${ }^{13} \mathrm{C}_{4}-\mathrm{PFBA},{ }^{13} \mathrm{C}_{5}-\mathrm{PFPeA},{ }^{13} \mathrm{C}_{5}-\mathrm{PFH} \mathrm{XA},{ }^{13} \mathrm{C}_{4}-\mathrm{PFHpA}$, ${ }^{13} \mathrm{C}_{8}$-PFOA, ${ }^{13} \mathrm{C}_{9}$-PFNA, ${ }^{13} \mathrm{C}_{6}$-PFDA, ${ }^{13} \mathrm{C}_{7}$-PFUnA, ${ }^{13} \mathrm{C}_{2}$-PFDoA, ${ }^{13} \mathrm{C}_{2}$-PFTeDA, ${ }^{13} \mathrm{C}_{3}-\mathrm{PFBS}$, ${ }^{13} \mathrm{C}_{3}$-PFHxS, ${ }^{13} \mathrm{C}_{8}$-PFOS, ${ }^{13} \mathrm{C}_{8}$-FOSA, $\mathrm{d}_{3}$-MeFOSA, $\mathrm{d}_{5}$-EtFOSA, $\mathrm{d}_{3}$-MeFOSAA, $\mathrm{d}_{5}$-EtFOSAA, ${ }^{13} \mathrm{C}_{2}-6: 2-\mathrm{FtS}$, and ${ }^{13} \mathrm{C}_{2}-8: 2-\mathrm{FtS}$ were obtained from Wellington Laboratories (Guelph, $\mathrm{ON}$, Canada). Perfluorooctane amidoakyl ammonium salt (N-TAmP-FOAd, obtained from the Beijing Surfactant Institute, Peking, China) was also used as an internal standard for correcting some of the positive mode PFAS. Further details on target analytes and internal standards are provided in Tables S1-S2. The mass-labelled performance internal standard solution MPFACC-IS $\left({ }^{13} \mathrm{C}_{3}\right.$-PFBA, ${ }^{13} \mathrm{C}_{2}$-PFOA, ${ }^{13} \mathrm{C}_{2}$-PFDA, ${ }^{13} \mathrm{C}_{4}$-PFOS $)$ was purchased from Wellington Laboratories (Guelph, ON, Canada).

HPLC water, HPLC water containing $0.1 \%$ formic acid, methanol, and acetonitrile of HPLC grade or Optima HPLC grade were purchased from Fisher Scientific (Whitby, ON, Canada). Ammonium acetate (purity $\geq 98 \%$ ), ammonium hydroxide (28-30\%, $\mathrm{NH}_{3}$ basis), and formic acid (reagent grade, purity $\geq 95 \%$ ) were acquired from Sigma-Aldrich (St. Louis, MO, USA). Nitrogen $\left(\mathrm{N}_{2}\right)$ (purity 99.998\%) was from MEGS Inc. (St-Laurent, QC, Canada). Supelclean ENVI-Carb cartridges (500 mg/6 mL) were obtained from Supelco (Bellefonte, PA, USA). The high-speed LP Vortex Mixer was from Fisher Scientific (Whitby, ON, Canada). 
Text S2. Targeted PFAS analysis.

Upon reception at the laboratory, dry organic solid wastes were crushed with mortar and pestle and passed through a 2-mm mesh sieve, except for sample \#007 (combustion cinders). Samples were re-homogenized with a stainless-steel spatula prior to aliquoting. A sample dry weight of $0.1 \mathrm{~g}$ (for municipal wastewater sludge) or $0.5 \mathrm{~g}$ (for other organic solid wastes) was introduced in a 15- $\mathrm{mL}$ polypropylene tube, and surrogate internal standards were spiked to the samples. After a wait time of $1 \mathrm{~h}$, solvent extraction proceeded as follows. Samples were submitted to three extraction cycles, each consisting in the addition of $4 \mathrm{~mL}$ of organic solvent, high-speed vortexing (0.5 min, $3200 \mathrm{rpm}$ ), ultrasonication (20 min), centrifugation (5 min, 5000 rpm), and transfer of the supernatant to a collecting 15-mL PP tube. The first two cycles involved $\mathrm{MeOH}$ containing $10 \mathrm{mM} \mathrm{NH}_{4} \mathrm{OH}$ as extractant, while the third cycle used $\mathrm{MeOH}$ containing $100 \mathrm{mM} \mathrm{CH}_{3} \mathrm{COONH}_{4}$. After a final rinse of solid materials with $2 \mathrm{~mL}$ of $\mathrm{MeOH}$ containing $100 \mathrm{mM} \mathrm{CH}_{3} \mathrm{COONH}_{4}$, the combined extract $(\sim 13.5 \mathrm{~mL})$ was concentrated to $<5$ $\mathrm{mL}$ under a gentle $\mathrm{N}_{2}$ stream and mild heating $\left(40-45^{\circ} \mathrm{C}\right)$. Extracts were then passed through Supelclean ENVI-Carb $(500 \mathrm{mg} / 6 \mathrm{~mL})$ cartridges previously conditioned with $\mathrm{MeOH}$, and the cartridges were rinsed with $4 \mathrm{~mL}$ of $\mathrm{MeOH}$. The extracts were concentrated to $\sim 1 \mathrm{~mL}$ and injection internal standards were spiked to the samples. After brief vortexing and centrifugation (5 min, $5000 \mathrm{rpm}$ ), a $180-\mu \mathrm{L}$ aliquot of the extract was transferred to a $250-\mu \mathrm{L}$ PP LC-MS vial and $20 \mu \mathrm{L}$ of HPLC-grade water were added.

Extracts were analyzed by ultra-high-performance liquid chromatography coupled to highresolution mass spectrometry (UHPLC-HRMS) through a polarity-switching electrospray ionization source (Munoz et al. 2018). The injection volume was set at $10 \mu \mathrm{L}$. Analyte separation was performed using a Thermo Hypersil Gold C18 column $(100 \mathrm{~mm} \times 2.1 \mathrm{~mm}$, $1.9 \mu \mathrm{m}$ particle size $)$. A Thermo Hypercarb column $(20 \mathrm{~mm} \times 2.1 \mathrm{~mm}, 7 \mu \mathrm{m}$ particle size $)$ was used as a PFAS trap column. Details on chromatographic mobile phases, elution gradient program, and other method settings are provided in Table S7. The Q-Exactive Orbitrap mass spectrometer (Thermo Fisher Scientific, Waltham, MA, USA) was operated in full scan MS mode for PFAS quantitation (scan range: $\mathrm{m} / \mathrm{z}$ 150-1000; resolution: 70,000 FWHM at $\mathrm{m} / \mathrm{z}$ 200).

The identification of target analytes relied on matching retention times $( \pm 0.1 \mathrm{~min})$ with certified standards, peak intensities superior to the set threshold (height $\geq 1 \mathrm{E} 4$ ), and exact mass accuracy with a tolerance of $\pm 10 \mathrm{ppm}$. Procedural blanks $(n=6)$ presented detectable levels of PFBA (0.24 $\mu \mathrm{g} / \mathrm{kg})$, PFOA (0.014 $\mu \mathrm{g} / \mathrm{kg})$, PFOS $(0.007 \mu \mathrm{g} / \mathrm{kg})$ and 6:2 FTSA $(0.012 \mu \mathrm{g} / \mathrm{kg})$. Determination coefficients $\left(R^{2}\right)$ of inverse-weighted linear regressions were between 0.9940 and 0.9999 (SI Table S8). Accuracy of quality control spikes $(n=4)$ was between $87-118 \%$, 
intraday precision $(\mathrm{RSD} \%)(\mathrm{n}=4)$ between $0.4-13 \%$, and interday precision $(\mathrm{n}=12)$ between $1.2-21 \%$ (SI Table S8). As an additional control of whole-method precision, a subset of the received samples was prepared and analyzed in duplicate.

Text S3. Occurrence of perfluoroalkyl acids in organic waste products.

Agricultural OWP $(n=21)$ presented consistently low PFAA levels ( $\sum$ PFAAs in samples with PFAS detections: $0.043-1.3 \mu \mathrm{g} / \mathrm{kg}$ ), independently of the particular site and sampling year. Few studies have investigated PFAS occurrence in animal-derived wastes, or the data available refer to mixed biowastes without the possibility of decorrelating the contribution of specific products (Suominen et al. 2014).

In urban OWP ( $\mathrm{n}=24)$, maximum $\Sigma$ PFAA concentrations varied as follows according to product type: municipal sludge $(370 \mu \mathrm{g} / \mathrm{kg}) \sim$ compost of green wastes and sewage sludge $(180 \mu \mathrm{g} / \mathrm{kg})>$ compost of residual municipal solid wastes $(94 \mu \mathrm{g} / \mathrm{kg})>>$ compost of municipal biowastes $(10 \mu \mathrm{g} / \mathrm{kg}) \sim$ digestate of urban wastes $(0.76 \mu \mathrm{g} / \mathrm{kg})$. Overall, PFOS and PFOA presented the highest detection rates at $100 \%$ and $96 \%$, respectively. PFOS was also predominant in the PFAA compositional profile, representing on average $57 \%$ of the $\Sigma$ PFAA. Other notable PFAAs included PFOA (15\% of $\Sigma$ PFAA), PFHxA (6.3\%), PFDA (4.8\%), and PFDoA (2.3\%). Another 27 PFAAs combined made less than $15 \%$ of the summed PFAAs. On average, perfluoroalkyl acids with an even number of carbon atoms were more abundant than their vicinal $\left(C_{n-1}\right.$ or $\left.C_{n+1}\right)$ odd-numbered analogs. For instance, PFDA (C10; $4.8 \%$ of $\left.\Sigma P F A A\right)$ was more abundant than PFNA (C9; $0.95 \%)$ or PFUnA $(\mathrm{C} 11 ; 0.96 \%)$. A similar trend is noted for perfluoroalkane sulfonates (PFSAs) and perfluorocarboxylates (PFCAs) in 4 of 6 source biosolids analyzed by Sepulvado et al. (2011) for C5-C14 PFCAs in 7 of 10 US municipal organic solid waste composts analyzed by Choi et al. (2019) and is also apparent in national biosolid inventories conducted in Australia and the US (Gallen et al. 2016; Venkatesan \& Halden 2013). For PFSAs, this likely reflects the composition of ECF-based products, including AFFFs, where C4, C6 and C8 PFSAs and their precursors are more abundant than odd-chain analogs.

The time series of historical WWTP sludge samples illustrates a gradual build up of PFOS: concentrations were low in the 1976-1980 ( 12 $\mu \mathrm{g} / \mathrm{kg})$ but had increased by an order of magnitude in the mid-1990s $(\sim 120 \mu \mathrm{g} / \mathrm{kg})$, consistent with the steep increase in global PFOS/POSF production over the 1970-1990 period (Paul et al. 2009). 


\section{SI Tables}

Table S1. Surrogate internal standards used in this study, including acronyms, names, $\mathrm{m} / \mathrm{z}$, ion mode, and supplier company.

\begin{tabular}{|c|c|c|c|c|}
\hline Acronym & Name & $m / z$ & Ion mode & Supplier \\
\hline${ }^{13} \mathrm{C}_{4}$-PFBA & Perfluoro-n- $\left[1,2,3,4-{ }^{13} \mathrm{C}_{4}\right]$ butanoic acid & 216.99177 & ESI- & Wellington Labs \\
\hline${ }^{13} \mathrm{C}_{5}$-PFPeA & Perfluoro-n- $\left[{ }^{13} \mathrm{C}_{5}\right]$ pentanoic acid & 267.99345 & ESI- & Wellington Labs \\
\hline${ }^{13} \mathrm{C}_{5}-\mathrm{PFHxA}$ & Perfluoro-n-[1,2,3,4,6- $\left.{ }^{13} \mathrm{C}_{5}\right]$ hexanoic acid & 317.99046 & ESI- & Wellington Labs \\
\hline${ }^{13} \mathrm{C}_{4}-\mathrm{PFHpA}$ & Perfluoro-n-[1,2,3,4-13 $\left.\mathrm{C}_{4}\right]$ heptanoic acid & 366.98249 & ESI- & Wellington Labs \\
\hline${ }^{13} \mathrm{C}_{8}$-PFOA & Perfluoro-n- $\left[{ }^{13} \mathrm{C}_{8}\right]$ octanoic acid & 420.99272 & ESI- & Wellington Labs \\
\hline${ }^{13} C_{9}$-PFNA & Perfluoro-n- $\left[{ }^{13} \mathrm{C}_{9}\right]$ nonanoic acid & 471.99288 & ESI- & Wellington Labs \\
\hline${ }^{13} \mathrm{C}_{6}$-PFDA & Perfluoro-n- $\left[1,2,3,4,5,6-{ }^{-13} \mathrm{C}_{6}\right]$ decanoic acid & 518.97962 & ESI- & Wellington Labs \\
\hline${ }^{13} C_{7}-$ PFUnA & Perfluoro-n-[1,2,3,4,5,6,7- $\left.{ }^{13} \mathrm{C}_{7}\right]$ undecanoic acid & 569.97978 & ESI- & Wellington Labs \\
\hline${ }^{13} C_{2}$-PFDoA & Perfluoro-n- $\left[1,2-{ }^{13} \mathrm{C}_{2}\right]$ dodecanoic acid & 614.95981 & ESI- & Wellington Labs \\
\hline${ }^{13} C_{2}$-PFTeDA & Perfluoro-n-[1,2- $\left.{ }^{13} \mathrm{C}_{2}\right]$ tetradecanoic acid & 714.95342 & ESI- & Wellington Labs \\
\hline${ }^{13} \mathrm{C}_{3}$-PFBS & Perfluoro-1-[2,3,4- $\left.{ }^{13} \mathrm{C}_{3}\right]$ butanesulfonate & 301.95251 & ESI- & Wellington Labs \\
\hline${ }^{13} \mathrm{C}_{3}$-PFHxS & Perfluoro-1-[1,2,3- $\left.-{ }^{13} \mathrm{C}_{3}\right]$ hexanesulfonate & 401.94612 & ESI- & Wellington Labs \\
\hline${ }^{13} \mathrm{C}_{8}$-PFOS & Perfluoro-1-[ $\left.{ }^{13} \mathrm{C}_{8}\right]$ octanesulfonate & 506.95641 & ESI- & Wellington Labs \\
\hline${ }^{13} \mathrm{C}_{8}-\mathrm{FOSA}$ & Perfluoro-1- $\left[{ }^{13} \mathrm{C}_{8}\right]$ octanesulfonamide & 505.97249 & ESI- & Wellington Labs \\
\hline$d_{3}-\mathrm{N}-\mathrm{MeFOSA}$ & $\mathrm{N}$-methyl- $\mathrm{d}_{3}$-perfluoro-1-octanesulfonamide & 514.98013 & ESI- & Wellington Labs \\
\hline$d_{5}-\mathrm{N}-\mathrm{EtFOSA}$ & $\mathrm{N}$-ethyl- $\mathrm{d}_{5}$-perfluoro-1-octanesulfonamide & 531.00830 & ESI- & Wellington Labs \\
\hline $\mathrm{d}_{3}-\mathrm{N}-\mathrm{MeFOSAA}$ & $\mathrm{N}$-methyl-d $\mathrm{d}_{3}$-perfluoro-1-octanesulfonamidoacetic acid & 572.98561 & ESI- & Wellington Labs \\
\hline $\mathbf{d}_{5}$-N-EtFOSAA & $\mathrm{N}$-ethyl-d $\mathrm{d}_{5}$-perfluoro-1-octanesulfonamidoacetic acid & 589.01382 & ESI- & Wellington Labs \\
\hline${ }^{13} C_{2}-6: 2$ FTSA & $1 \mathrm{H}, 1 \mathrm{H}, 2 \mathrm{H}, 2 \mathrm{H}$-perfluoro-1-[1,2-13 $\left.\mathrm{C}_{2}\right]$-octane sulfonate & 428.97537 & ESI- & Wellington Labs \\
\hline${ }^{13} \mathrm{C}_{2}-8: 2$ FTSA & $1 \mathrm{H}, 1 \mathrm{H}, 2 \mathrm{H}, 2 \mathrm{H}$-perfluoro-1-[1,2- $\left.{ }^{13} \mathrm{C}_{2}\right]-$ decane sulfonate & 528.96898 & ESI- & Wellington Labs \\
\hline${ }^{13} \mathrm{C}_{4}-6: 2$ diPAP & ${ }^{13} \mathrm{C}_{4}$-Bis- $(1 \mathrm{H}, 1 \mathrm{H}, 2 \mathrm{H}, 2 \mathrm{H}$-perfluorooctyl)phosphate & 792.98792 & ESI- & Wellington Labs \\
\hline TAmPr-FOAd (PFOAAmS) & Perfluorooctane amidoalkyl ammonium & 513.10176 & $\mathrm{ESI}+$ & Fluobon \\
\hline
\end{tabular}


Table S2. Acronym, name, and supplier of the 46 target PFAS with certified standards.

\begin{tabular}{|c|c|c|}
\hline Acronym & Name & Supplier \\
\hline PFPrA & Perfluoropropanoate & Sigma \\
\hline PFBA & Perfluorobutanoate & Wellington Labs \\
\hline PFPeA & Perfluoropentanoate & Wellington Labs \\
\hline PFHXA & Perfluorohexanoate & Wellington Labs \\
\hline PFHpA & Perfluoroheptanoate & Wellington Labs \\
\hline PFOA & Perfluorooctanoate & Wellington Labs \\
\hline PFNA & Perfluorononanoate & Wellington Labs \\
\hline PFDA & Perfluorodecanoate & Wellington Labs \\
\hline PFUnA & Perfluoroundecanoate & Wellington Labs \\
\hline PFDoA & Perfluorododecanoate & Wellington Labs \\
\hline PFTrDA & Perfluorotridecanoate & Wellington Labs \\
\hline PFTeDA & Perfluorotetradecanoate & Wellington Labs \\
\hline PFPrS & Perfluoropropane sulfonate & Wellington Labs \\
\hline PFBS & Perfluorobutane sulfonate & Wellington Labs \\
\hline PFPeS & Perfluorohexane sulfonate & Wellington Labs \\
\hline PFHxS & Perfluorohexane sulfonate & Wellington Labs \\
\hline PFHpS & Perfluoroheptane sulfonate & Wellington Labs \\
\hline PFOS & Perfluorooctane sulfonate & Wellington Labs \\
\hline PFDS & Perfluorodecane sulfonate & Wellington Labs \\
\hline FBSA & Perfluorobutane sulfonamide & Wellington Labs \\
\hline FHxSA & Perfluorohexane sulfonamide & Wellington Labs \\
\hline FOSA & Perfluorooctane sulfonamide & Wellington Labs \\
\hline MeFOSA & $\mathrm{N}$-methyl-perfluorooctane sulfonamide & Wellington Labs \\
\hline EtFOSA & $\mathrm{N}$-ethyl-perfluorooctane sulfonamide & Wellington Labs \\
\hline FOSAA & Perfluorooctane sulfonamidoacetic acid & Wellington Labs \\
\hline MeFOSAA & $\mathrm{N}$-methyl-perfluorooctane sulfonamidoacetic acid & Wellington Labs \\
\hline EtFOSAA & $\mathrm{N}$-ethyl-perfluorooctane sulfonamidoacetic acid & Wellington Labs \\
\hline $3: 3$ acid & $3: 3$ fluorotelomer carboxylate & Synquest \\
\hline $5: 3$ acid & $5: 3$ fluorotelomer carboxylate & DuPont USA \\
\hline $7: 3$ acid & $7: 3$ fluorotelomer carboxylate & DuPont USA \\
\hline $4: 2$ FTSA & $4: 2$ fluorotelomer sulfonate & Wellington Labs \\
\hline $6: 2$ FTSA & $6: 2$ fluorotelomer sulfonate & Wellington Labs \\
\hline 8:2 FTSA & 8:2 fluorotelomer sulfonate & Wellington Labs \\
\hline $6: 2$ diPAP & Bis- $(1 \mathrm{H}, 1 \mathrm{H}, 2 \mathrm{H}, 2 \mathrm{H}$-perfluorooctyl)phosphate & Wellington Labs \\
\hline $6: 2$ Cl-PFESA & $6: 2$ chlorinated perfluoroalkylether sulfonate & Wellington Labs \\
\hline 8:2 Cl-PFESA & $8: 2$ chlorinated perfluoroalkylether sulfonate & Wellington Labs \\
\hline PFECHS & Perfluoro-4-ethylcyclohexane sulfonate & Wellington Labs \\
\hline AmPr-FHxSA (PFHxSAm) & Perfluorohexane sulfonamidopropyl amine & Wellington Labs \\
\hline AmPr-FOSA (PFOSAm) & Perfluorooctane sulfonamidoalkyl amine & Fluobon \\
\hline T-AmPr-FHxSA (PFHxSAmS) & Perfluorohexane sulfonamidopropyl ammonium & Wellington Labs \\
\hline T-AmPr-FOSA (PFOSAmS) & Perfluorooctane sulfonamidopropyl ammonium & Fluobon \\
\hline CMeAmPr-FOAd (PFOAB) & Perfluorooctane amidopropyl betaine & Fluobon \\
\hline CMeAmPr-FOSA (PFOSB) & Perfluorooctane sulfonamidopropyl betaine & Fluobon \\
\hline OAmPr-FOAd (PFOANO) & Perfluorooctane amidopropyl amine oxide & Fluobon \\
\hline OAmPr-FOSA (PFOSNO) & Perfluorooctane sulfonamidopropyl amine oxide & Fluobon \\
\hline 6:2 FTSA-PrB (6:2 FTAB) & 6:2 fluorotelomer sulfonamidopropyl betaine & Wellington Labs \\
\hline
\end{tabular}


Table S3. Samples of organic waste products (OWP) per origin (urban, livestock manure, industry), with number of replicates per OWP sample, sites, and year of collection.

\begin{tabular}{|c|c|c|c|c|}
\hline OWP (code) & $\begin{array}{l}\text { Samples } \\
\text { (n) }\end{array}$ & Replicates & Sites & Year of collection \\
\hline \multicolumn{5}{|l|}{ Urban } \\
\hline Sewage sludge (SLU) & 10 & 3 (3 sites) ; 1 (2 sites) & 4 sites (Grand-Est ${ }^{\star}$, Nouvelle-Aquitaine, Réunion) & $\begin{array}{l}\text { 1976, 1980, 1978, 1985, 1993, 1996, } \\
1998,2009,2016,2017\end{array}$ \\
\hline Compost of green wastes and sewage sludge (C-GWS) & 6 & 3 (3 sites) ; 1 (1 site) & 4 sites (Grand-Est* ${ }^{*}$, lle-de-France, Réunion) & 1996, 2009, 2011, 2013, 2016, 2017 \\
\hline Compost of municipal biowastes (C-BIOW) & 4 & 3 (2 sites) & 2 sites (Grand-Est, lle-de-France) & 2009, 2011, 2013, 2016 \\
\hline Compost of residual municipal solid waste (C-MSW) & 3 & 3 (2 sites) & 2 sites (Grand-Est, lle-de-France) & $2011,2013,2016$ \\
\hline Digestate of urban wastes (DIG-UW) & 1 & 3 (1 site) & 1 site (Grand-Est) & 2016 \\
\hline \multicolumn{5}{|l|}{ Livestock manure } \\
\hline Farmyard manure of dairy cattle (FYM-DC) & 6 & 3 (2 sites) ; 1 (1 site) & 3 sites (Bretagne, Grand-Est, Ile-de-France) & $2011,2013,2014,2016,2018$ \\
\hline Pig slurry (PS) & 4 & 3 (1 site) ; 1 (1 site) & 2 sites (Bretagne, Réunion) & 2014, 2016, 2017, 2018 \\
\hline Poultry manure (PM) & 4 & 3 (1 site) ; 1 (1 site) & 2 sites (Bretagne, Réunion) & $2014,2016,2017,2018$ \\
\hline Compost of farmyard manure of dairy cattle (C-FYM-DC) & 1 & 3 (1 site) & 1 site (Grand-Est) & 2016 \\
\hline Compost of farmyard manure of pigs (C-FYM-P) & 3 & 1 (1 site) & 1 site (Bretagne) & 2014, 2016, 2018 \\
\hline Digestate of pig slurry (DIG-PS) & 3 & 1 (1 site) & 1 site (Bretagne) & 2014, 2016, 2018 \\
\hline \multicolumn{5}{|l|}{ Industry } \\
\hline Paper sludge (PSLU) & 1 & 1 (1 site) & 1 site (Grand-Est) & 1996 \\
\hline Ashes (ASH) & 1 & 1 (1 site) & 1 site (Grand-Est) & 1996 \\
\hline
\end{tabular}


Table S4. Samples of organic waste products (OWP), with the following metadata presented: Organic waste product name and code, origin, year of production, experimental site where it is studied (La Bouzule - Grand-Est, Couhins - Nouvelle-Aquitaine, EFELE - Bretagne, PROspective Grand-Est, QualiAgro - Ile-de-France, La Réunion - French Overseas), treatment process, raw materials, and number of replicates per sample.

\begin{tabular}{|c|c|c|c|c|c|c|c|}
\hline Origin & OWP & $\begin{array}{l}\text { OWP } \\
\text { code }\end{array}$ & Year & Site & Process & Raw material & Replicates \\
\hline Urban & Sewage sludge & SLU & 1976 & Couhins & Biological & Activated urban sewage sludge & 1 \\
\hline Urban & Sewage sludge & SLU & 1978 & Couhins & Biological & Activated urban sewage sludge & 1 \\
\hline Urban & Sewage sludge & SLU & 1980 & Couhins & Biological & Activated urban sewage sludge & 1 \\
\hline Urban & Sewage sludge & SLU & 1985 & Couhins & Biological & Activated urban sewage sludge & 1 \\
\hline Urban & Sewage sludge & SLU & 1993 & Couhins & Biological & Activated urban sewage sludge & 1 \\
\hline Urban & Sewage sludge & SLU & 1996 & La Bouzule & Biological+ deh* & Activated urban sewage sludge & 1 \\
\hline Urban & Sewage sludge & SLU & 1998 & Couhins & Biological & Activated urban sewage sludge & 1 \\
\hline Urban & Sewage sludge & SLU & 2009 & PROspective & Biological & Activated urban sewage sludge & 3 \\
\hline Urban & Sewage sludge & SLU & 2016 & PROspective & Biological & Activated urban sewage sludge & 3 \\
\hline Urban & Sewage sludge & SLU & 2017 & Réunion & Biological + deh* & Activated urban sewage sludge & 3 \\
\hline Urban & $\begin{array}{l}\text { Compost of green wastes and sewage } \\
\text { sludge }\end{array}$ & C-GWS & 1996 & La Bouzule & Composting & $\begin{array}{l}\text { Activated urban sewage sludge + Green } \\
\text { waste }\end{array}$ & 1 \\
\hline Urban & $\begin{array}{l}\text { Compost of green wastes and sewage } \\
\text { sludge } \\
\text { Compost of areen wastes and sewage }\end{array}$ & C-GWS & 2009 & PROspective & Composting & $\begin{array}{l}\text { Activated urban sewage sludge + Green } \\
\text { waste }\end{array}$ & 3 \\
\hline Urban & sludge & C-GWS & 2011 & QualiAgro & Composting & $\begin{array}{l}\text { Activate } \\
\text { waste }\end{array}$ & 2 \\
\hline Urban & $\begin{array}{l}\text { Compost of green wastes and sewage } \\
\text { sludge }\end{array}$ & C-GWS & 2013 & QualiAgro & Composting & $\begin{array}{l}\text { Activated urban sewage sludge }+ \text { Green } \\
\text { waste }\end{array}$ & 3 \\
\hline Urban & $\begin{array}{l}\text { Compost of green wastes and sewage } \\
\text { sludge }\end{array}$ & C-GWS & 2016 & PROspective & Composting & $\begin{array}{l}\text { Activated urban sewage sludge }+ \text { Green } \\
\text { waste }\end{array}$ & 3 \\
\hline Urban & $\begin{array}{l}\text { Compost of green wastes and sewage } \\
\text { sludge }\end{array}$ & C-GWS & 2017 & Réunion & Composting & $\begin{array}{l}\text { Activated urban sewage sludge }+ \text { Green } \\
\text { waste }\end{array}$ & 3 \\
\hline Urban & Compost of municipal biowastes & C-BIOW & 2009 & PROspective & Composting & Biowastes & 3 \\
\hline Urban & Compost of municipal biowastes & C-BIOW & 2011 & QualiAgro & Composting & Biowastes & 2 \\
\hline Urban & Compost of municipal biowastes & C-BIOW & 2013 & QualiAgro & Composting & Biowastes & 3 \\
\hline Urban & Compost of municipal biowastes & C-BIOW & 2016 & PROspective & Composting & Biowastes & 3 \\
\hline Urban & Compost of residual municipal solid waste & C-MSW & 1996 & La Bouzule & Composting & Municipal solid wastes & 3 \\
\hline Urban & Compost of residual municipal solid waste & C-MSW & 2011 & QualiAgro & Composting & Municipal solid wastes & 2 \\
\hline Urban & Compost of residual municipal solid waste & C-MSW & 2013 & QualiAgro & Composting & Municipal solid wastes & 3 \\
\hline Urban & Digestate of urban wastes & DIG-UW & 2016 & PROspective & Digestion & Cantine and restauration wastes & 3 \\
\hline Livestock manure & Farmyard manure of dairy cattle & FYM-DC & 2011 & QualiAgro & None & Cattle manure & 2 \\
\hline Livestock manure & Farmyard manure of dairy cattle & FYM-DC & 2013 & QualiAgro & None & Cattle manure & 3 \\
\hline Livestock manure & Farmyard manure of dairy cattle & FYM-DC & 2014 & EFELE & None & Cattle manure & 1 \\
\hline Livestock manure & Farmyard manure of dairy cattle & FYM-DC & 2016 & PROspective & None & Cattle manure & 3 \\
\hline Livestock manure & Farmyard manure of dairy cattle & FYM-DC & 2016 & EFELE & None & Cattle manure & 1 \\
\hline Livestock manure & Farmyard manure of dairy cattle & FYM-DC & 2018 & EFELE & None & Cattle manure & 1 \\
\hline
\end{tabular}




\begin{tabular}{|c|c|c|c|c|c|c|c|}
\hline Livestock manure & Pig slurry & PS & 2014 & EFELE & None & Pig slurry & 1 \\
\hline Livestock manure & Pig slurry & PS & 2016 & EFELE & None & Pig slurry & 1 \\
\hline Livestock manure & Pig slurry & PS & 2017 & Réunion & None & Pig slurry & 3 \\
\hline Livestock manure & Pig slurry & PS & 2018 & EFELE & None & Pig slurry & 1 \\
\hline Livestock manure & Poultry manure & PM & 2014 & EFELE & None & Poultry manure & 1 \\
\hline Livestock manure & Poultry manure & PM & 2016 & EFELE & None & Poultry manure & 1 \\
\hline Livestock manure & Poultry manure & PM & 2017 & Réunion & None & Poultry manure & 3 \\
\hline Livestock manure & Poultry manure & PM & 2018 & EFELE & None & Poultry manure & 1 \\
\hline Livestock manure & $\begin{array}{l}\text { Compost of farmyard manure of dairy } \\
\text { cattle }\end{array}$ & $\begin{array}{l}\text { C-FYM- } \\
\text { DC }\end{array}$ & 2016 & PROspective & Composting & Cattle manure & 3 \\
\hline Livestock manure & Compost of farmyard manure of pigs & C-FYM-P & 2014 & EFELE & Composting & Pig manure & 1 \\
\hline Livestock manure & Compost of farmyard manure of pigs & C-FYM-P & 2016 & EFELE & Composting & Pig manure & 1 \\
\hline Livestock manure & Compost of farmyard manure of pigs & C-FYM-P & 2018 & EFELE & Composting & Pig manure & 1 \\
\hline Livestock manure & Digestate of pig slurry & DIG-PS & 2014 & EFELE & Digestion & Pig slurry & 1 \\
\hline Livestock manure & Digestate of pig slurry & DIG-PS & 2016 & EFELE & Digestion & Pig slurry & 1 \\
\hline Livestock manure & Digestate of pig slurry & DIG-PS & 2018 & EFELE & Digestion & Pig slurry & 1 \\
\hline Industry & Paper sludge & PSLU & 1996 & La Bouzule & Liming & Mill sludge & 1 \\
\hline Industry & Ashes & $\mathrm{ASH}$ & 1996 & La Bouzule & Combustion & Ash & 1 \\
\hline
\end{tabular}

* dehydrated 
Table S5. Chemical properties of organic waste products (OWP) per site and year, with the following data presented: dry matter (DM; \% of fresh matter - FM), $\mathrm{pH}$, content of carbonates $\left(\mathrm{CaCO}_{3}\right)$, organic carbon $(\mathrm{OC})$, nitrogen $(\mathrm{N})$, phosphorus $\left(\mathrm{P}_{2} \mathrm{O}_{5}\right)$ and potassium $(\mathrm{K})$ (g per kilogram of dry matter).

\begin{tabular}{|c|c|c|c|c|c|c|c|c|c|c|}
\hline OWP & Site & Year & $\begin{array}{c}\text { DM } \\
\% \text { FM }\end{array}$ & $\mathrm{pH}$ & $\begin{array}{c}\mathrm{CaCO}_{3} \\
\mathrm{~g} / \mathrm{kg}\end{array}$ & $\begin{array}{l}\text { OC } \\
\mathrm{g} / \mathrm{kg}\end{array}$ & $\begin{array}{c}\mathbf{N}^{*} \\
\mathrm{~g} / \mathrm{kg}\end{array}$ & $\begin{array}{l}N^{\star *} \\
g / k g\end{array}$ & $\begin{array}{l}\mathbf{P}_{2} \mathbf{O}_{5} \\
\mathrm{~g} / \mathrm{kg}\end{array}$ & $\begin{array}{c}\mathbf{K} \\
\mathrm{g} / \mathrm{kg} \\
\end{array}$ \\
\hline SLU & Couhins & 1976 & na & na & na & 159 & 11.0 & na & 42.0 & 1.2 \\
\hline SLU & Couhins & 1978 & na & na & na & na & 27.1 & na & 42.0 & 1.7 \\
\hline SLU & Couhins & 1980 & na & na & na & 177 & na & na & 47.5 & 1.5 \\
\hline SLU & Couhins & 1985 & na & na & na & na & 19.8 & na & 25.0 & 8.3 \\
\hline SLU & Couhins & 1993 & na & na & na & na & 37.1 & na & 56.0 & 0.9 \\
\hline SLU & Bouzule & 1996 & 22 & 7.2 & na & 272 & na & 35.0 & 58.2 & 9.6 \\
\hline SLU & Couhins & 1998 & na & na & na & na & na & na & na & na \\
\hline SLU & PROspective & 2009 & 18.4 & 6.1 & 11.8 & 404 & 63.8 & na & 61.8 & 8.4 \\
\hline SLU & PROspective & 2016 & 18.8 & 7.2 & 12.2 & 373 & 58.2 & na & 62.1 & 6.8 \\
\hline SLU & Reunion & 2017 & 92.5 & 11.8 & na & 33 & 47.0 & 44.2 & 27.2 & 2.8 \\
\hline C-GWS & Bouzule & 1996 & 34 & 6.1 & na & 395 & na & 17.0 & 20.5 & 5.8 \\
\hline C-GWS & PROspective & 2009 & 55.1 & 7.6 & 50.9 & 250 & 28.2 & na & 34.9 & 16.5 \\
\hline C-GWS & QualiAgro & 2011 & 73.3 & 6.7 & 31.5 & 261 & 24.7 & na & 39.3 & 11.0 \\
\hline C-GWS & QualiAgro & 2013 & 67.2 & 7.7 & 44.1 & 257 & 22.5 & na & 27.7 & 17.1 \\
\hline C-GWS & PROspective & 2016 & 59.9 & 7.4 & 48.0 & 272 & 27.8 & na & 57.8 & 14.5 \\
\hline C-GWS & Reunion & 2017 & 62.7 & 8.2 & na & 29 & 36.7 & 27.1 & 22.1 & 7.3 \\
\hline C-BIOW & PROspective & 2009 & 57.1 & 8.5 & 57.9 & 242 & 22.3 & na & 10.8 & 25.1 \\
\hline C-BIOW & QualiAgro & 2011 & 80.9 & 7.5 & 22.5 & 295 & 23.8 & na & 11.8 & 21.5 \\
\hline C-BIOW & QualiAgro & 2013 & 76.2 & 7.8 & 19.8 & 232 & 17.4 & na & 8.6 & 23.4 \\
\hline C-BIOW & PROspective & 2016 & 56.0 & 8.2 & 117.0 & 259 & 19.5 & na & 13.5 & 21.9 \\
\hline C-MSW & Bouzule & 1996 & 67.5 & 7.6 & na & 210 & na & 11.0 & 5.1 & 10.3 \\
\hline C-MSW & QualiAgro & 2011 & 62.2 & 8.2 & 125.7 & 249 & 17.3 & na & 11.6 & 15.6 \\
\hline C-MSW & QualiAgro & 2013 & 63.6 & 7.9 & 62.8 & 322 & 15.8 & na & 8.8 & 10.0 \\
\hline DIG-UW & PROspective & 2018 & 6.6 & 8.8 & 76.0 & 368 & 90.7 & na & 36.1 & 53.0 \\
\hline FYM-DC & QualiAgro & 2011 & 43.8 & 9.4 & 64.3 & 199 & 15.7 & na & 11.8 & 34.8 \\
\hline FYM-DC & QualiAgro & 2013 & 33.9 & 8.8 & 45.7 & 355 & 19.7 & na & 15.7 & 32.3 \\
\hline FYM-DC & EFELE & 2014 & 25.2 & 9.8 & na & 394 & na & 5.2 & 12.3 & 38.0 \\
\hline FYM-DC & PROspective & 2016 & 28.9 & 9.4 & 16.4 & 415 & 15.8 & na & 10.8 & 39.1 \\
\hline FYM-DC & EFELE & 2016 & 24.5 & 9.8 & na & 380 & na & 5.4 & 13.0 & 39.7 \\
\hline FYM-DC & EFELE & 2018 & 23.1 & 9.4 & na & 337 & na & 6.1 & 12.0 & 41.2 \\
\hline PS & EFELE & 2014 & 6.2 & 10.0 & na & 334 & na & 5.8 & 63.8 & 81.3 \\
\hline PS & EFELE & 2016 & 7.9 & 9.7 & na & 393 & na & 5.9 & 46.4 & 49.3 \\
\hline PS & Reunion & 2017 & 7.5 & 7.5 & na & 37 & 32.3 & 3.3 & 27.9 & 31.8 \\
\hline PS & EFELE & 2018 & 8.0 & 8.5 & na & 404 & na & 6.2 & 44.6 & 43.1 \\
\hline PM & EFELE & 2014 & 40.5 & 8.1 & na & 269 & na & 15.0 & 54.4 & 35.5 \\
\hline PM & EFELE & 2016 & 38.3 & 8.6 & na & 278 & na & 12.0 & 77.1 & 30.7 \\
\hline PM & Reunion & 2017 & 64.2 & 8.1 & na & 41 & 41.7 & 29.5 & 13.2 & 42.2 \\
\hline $\mathrm{PM}$ & EFELE & 2018 & 62.7 & 7.8 & na & 273 & na & 19.5 & 59.1 & 34.5 \\
\hline C-FYM-DC & PROspective & 2016 & 19.9 & 9.3 & 43.5 & 325 & 27.0 & na & 25.8 & 52.7 \\
\hline C-FYM-P & EFELE & 2014 & 24.3 & 8.6 & na & 370 & na & 7.0 & 32.6 & 30.5 \\
\hline C-FYM-P & EFELE & 2016 & 28.6 & 8.3 & na & 310 & na & 6.7 & 66.4 & 26.7 \\
\hline C-FYM-P & EFELE & 2018 & 23.3 & 8.3 & na & 348 & na & 6.9 & 37.2 & 35.2 \\
\hline DIG-PS & EFELE & 2014 & 5.7 & 9.4 & na & 348 & na & 6.2 & 48.5 & 86.6 \\
\hline DIG-PS & EFELE & 2016 & 2.5 & 9.8 & na & 360 & na & 3.1 & 71.4 & 51.6 \\
\hline DIG-PS & EFELE & 2018 & 4.9 & 9.4 & na & 346 & na & 5.2 & 58.9 & 53.9 \\
\hline PSLU & Bouzule & 1996 & 43.4 & 5.7 & na & 425 & na & 13.0 & 11.0 & 5.1 \\
\hline $\mathrm{ASH}$ & Bouzule & 1996 & 77.6 & 8.9 & na & 50 & na & 0.5 & 1.1 & 31.6 \\
\hline
\end{tabular}

* Dumas method and ** Kjeldhal method; na for not available 
Table S6. Average chemical properties of the organic waste products (OWP), with the following data presented: dry matter (DM; \% of fresh matter - FM), $\mathrm{pH}$, content of carbonates $\left(\mathrm{CaCO}_{3}\right)$, organic carbon $(\mathrm{OC})$, nitrogen $(\mathrm{N})$, phosphorus $\left(\mathrm{P}_{2} \mathrm{O}_{5}\right)$ and potassium $(\mathrm{K})$ (g per kilogram of dry matter).

\begin{tabular}{ccccccccc}
\hline OWP & $\begin{array}{c}\text { DM } \\
\% \mathrm{MB}\end{array}$ & $\mathbf{p H}$ & $\begin{array}{c}\mathrm{CaCO}_{3} \\
\mathrm{~g} / \mathrm{kg}\end{array}$ & $\begin{array}{c}\mathbf{O C} \\
\mathbf{g} / \mathrm{kg}\end{array}$ & $\begin{array}{c}\mathbf{N}^{*} \\
\mathrm{~g} / \mathrm{kg}\end{array}$ & $\begin{array}{c}\mathbf{N}^{\star *} \\
\mathrm{~g} / \mathrm{kg}\end{array}$ & $\begin{array}{c}\mathbf{P}_{2} \mathbf{O}_{5} \\
\mathrm{~g} / \mathrm{kg}\end{array}$ & $\begin{array}{c}\mathbf{K} \\
\mathrm{g} / \mathrm{kg}\end{array}$ \\
\hline SLU & $54.1 \pm 41.1$ & $8.1 \pm 2.2$ & $12 \pm 0.3$ & $235 \pm 128$ & $47 \pm 32$ & $39.6 \pm 6.5$ & $43.1 \pm 17.8$ & $4.3 \pm 3.5$ \\
C-GWS & $63.6 \pm 6.9$ & $7.3 \pm 0.8$ & $43.6 \pm 8.5$ & $244 \pm 119$ & $28 \pm 5.4$ & $22.1 \pm 7.2$ & $33.7 \pm 13.8$ & $12 \pm 4.8$ \\
C-BIOW & $67.5 \pm 12.9$ & $8 \pm 0.4$ & $54.3 \pm 45.3$ & $257 \pm 28$ & $20.8 \pm 2.9$ & na & $11.2 \pm 2$ & $23 \pm 1.6$ \\
C-MSW & $62.9 \pm 1$ & $7.9 \pm 0.3$ & $94.2 \pm 44.5$ & $260 \pm 57$ & $16.5 \pm 1.1$ & na & $8.5 \pm 3.3$ & $12 \pm 3.2$ \\
DIG-UW & 6.6 & 8.8 & 76.0 & 368 & 90.7 & na & 36.1 & 53.0 \\
FYM-DC & $29.9 \pm 7.9$ & $9.4 \pm 0.4$ & $42.1 \pm 24.1$ & $347 \pm 77$ & $17.1 \pm 2.3$ & $5.6 \pm 0.5$ & $12.6 \pm 1.7$ & $37.5 \pm 3.3$ \\
PS & $7.39 \pm 0.82$ & $8.9 \pm 1.2$ & na & $292 \pm 173$ & na & $5.3 \pm 1.3$ & $45.7 \pm 14.7$ & $51.4 \pm 21.2$ \\
PM & $51.4 \pm 13.9$ & $8.2 \pm 0.3$ & na & $215 \pm 116$ & na & $19 \pm 7.6$ & $51 \pm 27$ & $35.7 \pm 4.8$ \\
C-FYM-DC & 19.9 & 9.3 & 43.5 & 325 & 27.0 & na & 25.8 & 52.7 \\
C-FYM-P & $25.4 \pm 2.8$ & $8.4 \pm 0.2$ & na & $343 \pm 30$ & na & $6.9 \pm 0.1$ & $45.4 \pm 18.3$ & $30.8 \pm 4.3$ \\
DIG-PS & $4.4 \pm 1.7$ & $9.5 \pm 0.3$ & na & $351 \pm 8$ & na & $4.8 \pm 1.6$ & $59.6 \pm 11.5$ & $64 \pm 19.6$ \\
PSLU & 43.4 & 5.7 & na & 425 & na & 13.0 & 11.0 & 5.1 \\
ASH & 77.6 & 8.9 & na & 50 & na & 0.5 & 1.1 & 31.6 \\
\hline
\end{tabular}

* Dumas method and ** Kjeldhal method; na for not available 
Table S7. Details on the UHPLC-HRMS instrumental method for PFAS analysis.

\begin{tabular}{|c|c|c|c|}
\hline UHPLC-HRMS system & \multicolumn{3}{|c|}{$\begin{array}{l}\text { Dionex Ultimate } 3000 \text { UHPLC chain, heated electrospray ionization source (polarity } \\
\text { switching) } \\
\text { Thermo Q-Exactive Orbitrap high-resolution mass spectrometer }\end{array}$} \\
\hline Separation column & \multicolumn{3}{|c|}{ Thermo Hypersil Gold C18 column $(100 \mathrm{~mm} \times 2.1 \mathrm{~mm} ; 1.9 \mu \mathrm{m})$} \\
\hline Delay column & \multicolumn{3}{|c|}{ Thermo Hypercarb column $(20 \mathrm{~mm} \times 2.1 \mathrm{~mm} ; 7 \mu \mathrm{m})$} \\
\hline $\begin{array}{l}\text { Column oven } \\
\text { temperature }\end{array}$ & \multicolumn{3}{|l|}{$40^{\circ} \mathrm{C}$} \\
\hline UHPLC mobile phases & \multicolumn{3}{|c|}{$\begin{array}{l}\text { A: } 0.1 \% \text { formic acid in HPLC-water } \\
\text { B: } 0.1 \% \text { formic acid in ACN } \\
\text { Mobile phase flow rate } 550 \mu \mathrm{L} / \mathrm{min}\end{array}$} \\
\hline $\begin{array}{l}\text { Chromatographic } \\
\text { gradient }\end{array}$ & $\begin{array}{l}\text { Time (min) } \\
0.0 \\
7.0 \\
8.5 \\
12.5 \\
12.6 \\
14.6 \\
\end{array}$ & $\begin{array}{l}\% \mathrm{~A} \\
90 \\
27.5 \\
0 \\
0 \\
90 \\
90 \\
\end{array}$ & $\begin{array}{l}\% B \\
10 \\
72.5 \\
100 \\
100 \\
10 \\
10 \\
\end{array}$ \\
\hline Injection Volume & \multicolumn{3}{|l|}{$10 \mu \mathrm{L}$} \\
\hline Source & \multicolumn{3}{|c|}{$\begin{array}{l}\text { Sheath gas flow rate } 50 \text { a.u. } \\
\text { Aux gas flow rate } 15 \text { a.u. } \\
\text { Sweep gas flow rate } 0 \text { a.u. } \\
\text { Positive spray voltage }(|\mathrm{V}|) 3500 \\
\text { Negative spray voltage }(|\mathrm{V}|) 3000 \\
\text { Capillary temperature }\left({ }^{\circ} \mathrm{C}\right) 320 \\
\text { Vaporizer temperature }\left({ }^{\circ} \mathrm{C}\right) 350 \\
\text { S-lens RF level } 55\end{array}$} \\
\hline $\begin{array}{l}\text { Q-Exactive Orbitrap } \\
\text { settings }\end{array}$ & \multicolumn{3}{|c|}{$\begin{array}{l}\text { Full Scan MS mode } \\
\text { Scan range }(\mathrm{m} / \mathrm{z}) 150-1000 \\
\text { Resolution } 70,000 \text { at } \mathrm{m} / \mathrm{z} 200 \\
\text { AGC target } 3 \mathrm{e} 6 \\
\text { Max. Inject Time (ms) } 50 \\
\\
\text { PRM mode (MS/MS) } \\
\text { Variable PRM inclusion list } \\
\text { Variable NCE (normalized collision energy): } 20-50 \% \\
\text { Resolution } 17,500 \text { at } m / z 200 \\
\text { AGC target 2e5 } \\
\text { Max. Inject Time (ms) } 50\end{array}$} \\
\hline
\end{tabular}


Table S8. Instrumental performance, including method limits of detection $(\mu \mathrm{g} / \mathrm{kg}), R^{2}$, accuracy\% $(n=4)$ and corresponding intraday precision $(R S D \%, n=4)$, and interday precision $(\mathrm{RSD} \%, \mathrm{n}=12)$.

\begin{tabular}{|c|c|c|c|c|c|}
\hline & $\begin{array}{l}\mathrm{LOD}^{*} \\
(\mu \mathrm{g} / \mathrm{kg})\end{array}$ & $\mathrm{R}^{2}$ & $\begin{array}{l}\text { Accuracy\% } \\
(\mathrm{n}=4)\end{array}$ & $\begin{array}{l}\text { Intraday precision } \\
\text { RSD\% }(n=4)\end{array}$ & $\begin{array}{c}\text { Interday precision } \\
\text { RSD\% }(\mathrm{n}=12)\end{array}$ \\
\hline PFPrA & 0.25 & 0.9940 & 110.8 & 5.9 & 7.1 \\
\hline PFBA & 0.05 & 0.9989 & 117.9 & 2.4 & 3.7 \\
\hline PFPeA & 0.025 & 0.9998 & 105.3 & 2.1 & 1.7 \\
\hline PFHxA & 0.025 & 0.9997 & 105.4 & 1.4 & 1.6 \\
\hline PFHpA & 0.013 & 0.9999 & 105.3 & 1.8 & 2.0 \\
\hline PFOA & 0.011 & 0.9994 & 107.4 & 2.9 & 1.9 \\
\hline PFNA & 0.011 & 0.9994 & 106.0 & 1.1 & 1.4 \\
\hline PFDA & 0.01 & 0.9998 & 104.4 & 1.8 & 2.1 \\
\hline PFUnA & 0.007 & 0.9993 & 104.9 & 2.4 & 2.2 \\
\hline PFDoDA & 0.008 & 0.9990 & 109.0 & 1.7 & 1.7 \\
\hline PFTrDA & 0.008 & 0.9948 & 117.1 & 0.6 & 20.6 \\
\hline PFTeDA & 0.014 & 0.9990 & 109.6 & 0.5 & 1.7 \\
\hline PFPrS & 0.009 & 0.9998 & 102.8 & 1.9 & 5.1 \\
\hline PFBS & 0.007 & 0.9999 & 106.3 & 1.2 & 1.4 \\
\hline PFPeS & 0.006 & 0.9998 & 108.4 & 2.4 & 4.1 \\
\hline PFHxS & 0.006 & 0.9999 & 102.7 & 1.3 & 1.6 \\
\hline PFHpS & 0.006 & 0.9996 & 107.7 & 3.9 & 3.6 \\
\hline PFOS & 0.006 & 0.9996 & 105.3 & 1.8 & 1.2 \\
\hline PFDS & 0.006 & 0.9989 & 101.4 & 4.4 & 7.2 \\
\hline FBSA & 0.007 & 0.9992 & 92.7 & 2.7 & 8.0 \\
\hline $\mathrm{FHxSA}$ & 0.006 & 0.9995 & 98.6 & 2.5 & 8.5 \\
\hline FOSA & 0.005 & 0.9997 & 104.7 & 2.3 & 1.9 \\
\hline MeFOSA & 0.014 & 0.9984 & 105.7 & 4.5 & 3.7 \\
\hline EtFOSA & 0.013 & 0.9980 & 102.2 & 7.0 & 7.2 \\
\hline FOSAA & 0.025 & 0.9984 & 101.3 & 1.6 & 12.6 \\
\hline MeFOSAA & 0.1 & 0.9996 & 103.2 & 3.9 & 4.9 \\
\hline EtFOSAA & 0.1 & 0.9992 & 95.4 & 2.2 & 15.9 \\
\hline 3:3 Acid & 0.25 & 0.9988 & 98.6 & 5.2 & 12.7 \\
\hline 5:3 Acid & 0.1 & 0.9989 & 93.7 & 1.3 & 14.0 \\
\hline 7:3 Acid & 0.1 & 0.9979 & 112.7 & 7.4 & 18.1 \\
\hline 4:2 FTSA & 0.025 & 0.9991 & 103.8 & 8.0 & 6.8 \\
\hline 6:2 FTSA & 0.025 & 0.9999 & 101.7 & 1.6 & 1.3 \\
\hline 8:2 FTSA & 0.03 & 0.9988 & 106.8 & 1.2 & 1.2 \\
\hline $6: 2$ diPAP & 0.15 & 0.9994 & 105.8 & 12.5 & 6.9 \\
\hline 6:2 Cl-PFESA & 0.01 & 0.9992 & 101.5 & 3.5 & 4.8 \\
\hline 8:2 Cl-PFESA & 0.009 & 0.9995 & 103.4 & 2.7 & 12.2 \\
\hline PFECHS & 0.008 & 0.9985 & 98.1 & 1.1 & 3.4 \\
\hline AmPr-FHxSA & 0.01 & 0.9997 & 106.3 & 5.2 & 3.8 \\
\hline AmPr-FOSA & 0.023 & 0.9992 & 97.1 & 0.4 & 6.0 \\
\hline T-AmPr-FHxSA & 0.015 & 0.9983 & 86.6 & 2.7 & 5.9 \\
\hline T-AmPr-FOSA & 0.036 & 0.9980 & 89.6 & 3.1 & 4.0 \\
\hline CMeAmPr-FOAd & 0.021 & 0.9986 & 91.4 & 5.5 & 7.1 \\
\hline CMeAmPr-FOSA & 0.14 & 0.9990 & 92.9 & 6.9 & 13.2 \\
\hline OAmPr-FOAd & 0.021 & 0.9998 & 100.3 & 2.9 & 4.6 \\
\hline OAmPr-FOSA & 0.03 & 0.9987 & 100.5 & 3.6 & 7.9 \\
\hline 6:2 FTSA-PrB & 0.05 & 0.9994 & 101.1 & 6.0 & 6.3 \\
\hline
\end{tabular}

${ }^{*}$ LODs were calculated based on a sample intake of $500 \mathrm{mg}$ dry weight. 
Table S9. Accuracy\% of continued calibration verification (CCV) standards run along the LCMS batch sequence $(n=6)$.

\begin{tabular}{|c|c|c|c|}
\hline & Accuracy\% & $\operatorname{STDEV}(n=6)$ & RSD\% $(n=6)$ \\
\hline PFPrA & 101.4 & 5.6 & 5.5 \\
\hline PFBA & 108.5 & 2.8 & 2.6 \\
\hline PFPeA & 105.5 & 3.4 & 3.2 \\
\hline PFHXA & 101.4 & 2.7 & 2.7 \\
\hline PFHpA & 103.0 & 2.0 & 1.9 \\
\hline PFOA & 104.7 & 1.6 & 1.5 \\
\hline PFNA & 103.4 & 1.2 & 1.2 \\
\hline PFDA & 101.8 & 1.7 & 1.6 \\
\hline PFUnA & 102.0 & 1.5 & 1.4 \\
\hline PFDoDA & 105.8 & 1.0 & 0.9 \\
\hline PFTrDA & 107.2 & 12.5 & 11.7 \\
\hline PFTeDA & 106.1 & 1.6 & 1.5 \\
\hline PFPrS & 99.2 & 2.1 & 2.1 \\
\hline PFBS & 102.7 & 2.3 & 2.2 \\
\hline PFPeS & 102.5 & 4.5 & 4.4 \\
\hline PFHxS & 101.9 & 1.0 & 1.0 \\
\hline PFHpS & 95.5 & 3.5 & 3.6 \\
\hline PFOS & 105.3 & 1.6 & 1.6 \\
\hline PFDS & 103.2 & 8.9 & 8.6 \\
\hline FBSA & 100.5 & 12.7 & 12.6 \\
\hline FHxSA & 103.8 & 7.3 & 7.0 \\
\hline FOSA & 102.8 & 1.3 & 1.3 \\
\hline MeFOSA & 102.2 & 3.0 & 3.0 \\
\hline EtFOSA & 99.6 & 8.2 & 8.3 \\
\hline FOSAA & 112.8 & 4.8 & 4.3 \\
\hline MeFOSAA & 97.0 & 1.9 & 2.0 \\
\hline EtFOSAA & 107.4 & 8.6 & 8.0 \\
\hline 3:3 Acid & 108.6 & 3.4 & 3.2 \\
\hline 5:3 Acid & 110.7 & 6.8 & 6.2 \\
\hline 7:3 Acid & 121.0 & 17.1 & 14.1 \\
\hline 4:2 FTSA & 103.4 & 7.5 & 7.3 \\
\hline 6:2 FTSA & 101.6 & 2.3 & 2.2 \\
\hline 8:2 FTSA & 103.2 & 0.8 & 0.7 \\
\hline $6: 2$ diPAP & 100.0 & 2.0 & 2.0 \\
\hline 6:2 Cl-PFESA & 99.7 & 5.1 & 5.1 \\
\hline 8:2 Cl-PFESA & 99.0 & 12.5 & 12.6 \\
\hline PFECHS & 94.3 & 3.4 & 3.6 \\
\hline AmPr-FHxSA & 95.3 & 2.3 & 2.4 \\
\hline AmPr-FOSA & 101.7 & 4.2 & 4.1 \\
\hline T-AmPr-FHxSA & 81.3 & 1.3 & 1.6 \\
\hline T-AmPr-FOSA & 89.5 & 4.4 & 5.0 \\
\hline CMeAmPr-FOAd & 89.8 & 3.9 & 4.4 \\
\hline CMeAmPr-FOSA & 86.4 & 7.4 & 8.6 \\
\hline OAmPr-FOAd & 97.0 & 2.1 & 2.1 \\
\hline OAmPr-FOSA & 93.2 & 2.8 & 3.0 \\
\hline 6:2 FTSA-PrB & 95.0 & 5.7 & 6.0 \\
\hline
\end{tabular}


Table S10. Matrix spike recovery \% (average \pm STDEV) of target PFAS to 4 model organic solid waste products.

\begin{tabular}{|c|c|c|c|c|}
\hline & $\begin{array}{l}\text { Compost of municipal biowastes } \\
\qquad(\mathrm{n}=3)\end{array}$ & $\begin{array}{c}\text { Poultry litter } \\
(\mathrm{n}=3)\end{array}$ & $\begin{array}{l}\text { Farmyard manure of dairy cattle } \\
\qquad(\mathrm{n}=3)\end{array}$ & $\begin{array}{c}\text { Pig slurry } \\
(\mathrm{n}=3)\end{array}$ \\
\hline PFPrA & $101 \pm 7$ & $93 \pm 14$ & $98 \pm 7$ & $97 \pm 15$ \\
\hline PFBA & $107 \pm 6$ & $104 \pm 12$ & $91 \pm 0.4$ & $101 \pm 5$ \\
\hline PFPeA & $106 \pm 1$ & $98 \pm 16$ & $100 \pm 1$ & $94 \pm 6$ \\
\hline PFHxA & $104 \pm 8$ & $97 \pm 6$ & $96 \pm 1$ & $90 \pm 2$ \\
\hline PFHpA & $104 \pm 6$ & $100 \pm 1$ & $98 \pm 1$ & $101 \pm 3$ \\
\hline PFOA & $101 \pm 2$ & $97 \pm 2$ & $95 \pm 1$ & $98 \pm 3$ \\
\hline PFNA & $104 \pm 1$ & $98 \pm 1$ & $95 \pm 2$ & $96 \pm 3$ \\
\hline PFDA & $103 \pm 4$ & $100 \pm 3$ & $94 \pm 1$ & $95 \pm 2$ \\
\hline PFUnA & $99 \pm 3$ & $96 \pm 5$ & $94 \pm 1$ & $92 \pm 2$ \\
\hline PFDoDA & $102 \pm 3$ & $101 \pm 4$ & $95 \pm 2$ & $98 \pm 2$ \\
\hline PFTrDA & $108 \pm 1$ & $99 \pm 5$ & $95 \pm 2$ & $99 \pm 2$ \\
\hline PFTeDA & $100 \pm 2$ & $98 \pm 1$ & $95 \pm 1$ & $98 \pm 3$ \\
\hline PFPrS & $101 \pm 6$ & $96 \pm 3$ & $91 \pm 5$ & $91 \pm 5$ \\
\hline PFBS & $99 \pm 4$ & $96 \pm 2$ & $93 \pm 2$ & $96 \pm 2$ \\
\hline PFPeS & $99 \pm 9$ & $100 \pm 6$ & $96 \pm 3$ & $97 \pm 2$ \\
\hline PFHxS & $99 \pm 3$ & $98 \pm 4$ & $94 \pm 2$ & $97 \pm 2$ \\
\hline PFHpS & $101 \pm 5$ & $94 \pm 4$ & $96 \pm 3$ & $94 \pm 1$ \\
\hline PFOS & $103 \pm 2$ & $98 \pm 2$ & $96 \pm 1$ & $95 \pm 2$ \\
\hline PFDS & $98 \pm 3$ & $100 \pm 3$ & $91 \pm 2$ & $94 \pm 3$ \\
\hline FBSA & $105 \pm 5$ & $96 \pm 17$ & $94 \pm 2$ & $97 \pm 8$ \\
\hline FHxSA & $101 \pm 5$ & $101 \pm 2$ & $95 \pm 3$ & $95 \pm 1$ \\
\hline FOSA & $102 \pm 1$ & $107 \pm 5$ & $96 \pm 1$ & $97 \pm 5$ \\
\hline MeFOSA & $88 \pm 3$ & $82 \pm 5$ & $91 \pm 2$ & $93 \pm 5$ \\
\hline EtFOSA & $86 \pm 1$ & $82 \pm 3$ & $88 \pm 3$ & $89 \pm 4$ \\
\hline FOSAA & $95 \pm 3$ & $105 \pm 15$ & $82 \pm 1$ & $85 \pm 3$ \\
\hline MeFOSAA & $100 \pm 2$ & $94 \pm 4$ & $94 \pm 2$ & $90 \pm 1$ \\
\hline EtFOSAA & $98 \pm 3$ & $99 \pm 5$ & $95 \pm 3$ & $88 \pm 3$ \\
\hline 3:3 Acid & $82 \pm 15$ & $97 \pm 4$ & $93 \pm 5$ & $94 \pm 37$ \\
\hline 5:3 Acid & $105 \pm 2$ & $93 \pm 20$ & $91 \pm 2$ & $91 \pm 4$ \\
\hline 7:3 Acid & $94 \pm 7$ & $89 \pm 19$ & $92 \pm 2$ & $75 \pm 4$ \\
\hline $4: 2$ FTSA & $100 \pm 4$ & $101 \pm 6$ & $90 \pm 3$ & $101 \pm 7$ \\
\hline $6: 2$ FTSA & $100 \pm 2$ & $100 \pm 3$ & $94 \pm 0.3$ & $93 \pm 2$ \\
\hline 8:2 FTSA & $101 \pm 4$ & $99 \pm 4$ & $95 \pm 1$ & $93 \pm 2$ \\
\hline $6: 2 \mathrm{diPAP}$ & $102 \pm 0.5$ & $97 \pm 3$ & $99 \pm 5$ & $96 \pm 2$ \\
\hline 6:2 Cl-PFESA & $105 \pm 3$ & $96 \pm 5$ & $89 \pm 3$ & $92 \pm 3$ \\
\hline 8:2 Cl-PFESA & $103 \pm 3$ & $100 \pm 9$ & $94 \pm 4$ & $95 \pm 1$ \\
\hline PFECHS & $99 \pm 4$ & $99 \pm 2$ & $95 \pm 3$ & $96 \pm 2$ \\
\hline AmPr-FHxSA & $98 \pm 5$ & $105 \pm 6$ & $90 \pm 4$ & $106 \pm 5$ \\
\hline AmPr-FOSA & $100 \pm 3$ & $91 \pm 7$ & $90 \pm 3$ & $104 \pm 7$ \\
\hline T-AmPr-FHxSA & $111 \pm 2$ & $105 \pm 6$ & $93 \pm 3$ & $104 \pm 9$ \\
\hline T-AmPr-FOSA & $104 \pm 7$ & $101 \pm 6$ & $90 \pm 4$ & $99 \pm 9$ \\
\hline CMeAmPr-FOAd & $98 \pm 4$ & $105 \pm 7$ & $90 \pm 6$ & $86 \pm 6$ \\
\hline CMeAmPr-FOSA & $103 \pm 8$ & $92 \pm 19$ & $95 \pm 6$ & $93 \pm 11$ \\
\hline OAmPr-FOAd & $101 \pm 5$ & $106 \pm 7$ & $89 \pm 4$ & $89 \pm 6$ \\
\hline OAmPr-FOSA & $113 \pm 6$ & $98 \pm 3$ & $91 \pm 2$ & $100 \pm 6$ \\
\hline 6:2 FTSA-PrB & $112 \pm 12$ & $103 \pm 10$ & $94 \pm 4$ & $102 \pm 17$ \\
\hline
\end{tabular}


Table S11. PFAS concentrations $(\mu \mathrm{g} / \mathrm{kg}$ ) determined in NIST SRM 2781 (standard reference material of domestic sludge).

\begin{tabular}{lcc} 
& \multicolumn{2}{c}{ Concentration $(\mu \mathrm{g} / \mathrm{kg})$} \\
\cline { 2 - 3 } & $\begin{array}{c}\text { This study } \\
(\mathrm{n}=5)\end{array}$ & $\begin{array}{c}\text { Reference value } \\
\text { (NIST certificate) }\end{array}$ \\
\hline PFBA & $6.3 \pm 0.3$ & - \\
PFPeA & $6.6 \pm 0.4$ & - \\
PFHxA & $17 \pm 0.9$ & $13.0 \pm 2.0$ \\
PFHpA & $9.6 \pm 0.5$ & $7.96 \pm 1.50$ \\
PFOA & $25 \pm 1.8$ & $28.5 \pm 3.3$ \\
PFNA & $1.7 \pm 0.3$ & - \\
PFHxS & $7.6 \pm 0.3$ & $9.39 \pm 1.96$ \\
PFOS & $240 \pm 13$ & $225 \pm 41$ \\
PFDS & $230 \pm 11$ & - \\
FOSA & $7.4 \pm 1.3$ & $6.31 \pm 0.97$ \\
MeFOSAA & $52 \pm 3.2$ & - \\
EtFOSAA & $465 \pm 25$ & - \\
6:2 FTSA & $2.0 \pm 0.3$ & - \\
6:2 diPAP & $18 \pm 1.1$ & - \\
PFECHS & $2.7 \pm 0.6$ & - \\
T-AmPr-FOSA & $100 \pm 1.3$ & - \\
AmPr-FOSA & $2.0 \pm 0.4$ & - \\
\hline
\end{tabular}

Table S12. Summary of \% PFAS recovered by a secondary extraction on already solventextracted organic waste products, tested for six products with high PFAS content $\left(\Sigma_{46}\right.$ PFAS $>200 \mu \mathrm{g} / \mathrm{kg}$ ).

\begin{tabular}{rcccccc} 
& \multicolumn{6}{c}{ \% PFAS recovered by a secondary extraction to already extracted OWP } \\
\cline { 2 - 7 } & SLU $^{\text {a }}$ & C-MSW $^{\mathrm{b}}$ & C-GWS $^{\mathrm{c}}$ & C-MSW $^{\mathrm{d}}$ & C-GWS $^{\text {e }}$ & C-MSW $^{\mathrm{f}}$ \\
\cline { 2 - 7 } Min\% & 0.17 & 0.48 & 0.58 & 0.85 & 1.2 & 0.65 \\
Max\% & 1.8 & 3.0 & 6.4 & 6.0 & 9.0 & 4.2 \\
Mean\% & 0.88 & 1.43 & 1.73 & 2.07 & 3.25 & 2.10 \\
\hline
\end{tabular}

\footnotetext{
a Urban sewage sludge (La Bouzule); ${ }^{\text {b }}$ Compost of residual municipal solid waste (La Bouzule); ${ }^{\mathrm{c}}$ Compost of green wastes and sewage sludge (La Bouzule); ${ }^{\mathrm{C}}$ Compost of green wastes and sewage sludge (La Bouzule); ${ }^{\mathrm{d}}$ Compost of residual municipal solid waste (QualiAgro); ${ }^{\text {e }}$ Compost of green wastes and sewage sludge (QualiAgro); ${ }^{\dagger}$ Compost of residual municipal solid waste (PROspective).
} 
Table S13. Details on the 160 PFAS detected in the present study, including class, homolog acronym, ion formula, ion mode, theoretical $m / z$, observed $\mathrm{m} / \mathrm{z}$, exact mass error ( $\delta \mathrm{ppm})$, and reference calibrant for quantification or semi-quantification.

\begin{tabular}{|c|c|c|c|c|c|c|c|c|}
\hline Ana & Clas & Chemical class & Acronym & Ion formula & $\mathrm{m} / \mathrm{z}$ theoretica & $\mathrm{m} / \mathrm{z}$ measured & $\delta \mathrm{ppm}$ & $\begin{array}{l}\text { Reference calibrant for } \\
\text { quantification or semi- } \\
\text { quantification }\end{array}$ \\
\hline 1 & $\# 1$ & Perfluorocarboxylates (PFCAs) & PFPrA & $\mathrm{C}_{3} \mathrm{~F}_{5} \mathrm{O}_{2}^{-}$ & 162.98185 & 162.98164 & $\mid-1.3$ & PFPrA \\
\hline 2 & $\# 1$ & Perfluorocarboxylates (PFCAs) & PFBA & $\mathrm{C}_{4} \mathrm{~F}_{7} \mathrm{O}_{2}^{-}$ & 212.97865 & 212.97934 & 3.2 & PFBA \\
\hline 3 & $\# 1$ & Perfluorocarboxylates (PFCAs) & PFPeA & $\mathrm{C}_{5} \mathrm{~F}_{9} \mathrm{O}_{2}{ }^{-}$ & 262.97546 & 262.97659 & 4.3 & PFPeA \\
\hline 4 & $\# 1$ & Perfluorocarboxylates (PFCAs) & PFHXA & $\mathrm{C}_{6} \mathrm{~F}_{11} \mathrm{O}_{2}^{-}$ & 312.97226 & 312.97369 & 4.6 & PFHXA \\
\hline 5 & $\# 1$ & Perfluorocarboxylates (PFCAs) & PFHpA & $\mathrm{C}_{7} \mathrm{~F}_{13} \mathrm{O}_{2}^{-}$ & 362.96907 & 362.97043 & \begin{tabular}{|l|}
3.7 \\
\end{tabular} & PFHpA \\
\hline 6 & $\# 1$ & Perfluorocarboxylates (PFCAs) & PFOA & $\mathrm{C}_{8} \mathrm{~F}_{15} \mathrm{O}_{2}^{-}$ & 412.96588 & 412.96707 & 2.9 & PFOA \\
\hline 7 & $\# 1$ & Perfluorocarboxylates (PFCAs) & PFNA & $\mathrm{C}_{9} \mathrm{~F}_{17} \mathrm{O}_{2}^{-}$ & 462.96268 & 462.96399 & 2.8 & PFNA \\
\hline 8 & $\# 1$ & Perfluorocarboxylates (PFCAs) & PFDA & $\mathrm{C}_{10} \mathrm{~F}_{19} \mathrm{O}_{2}{ }^{-}$ & 512.95949 & 512.96039 & 1.8 & PFDA \\
\hline 9 & $\# 1$ & Perfluorocarboxylates (PFCAs) & PFUnA & $\mathrm{C}_{11} \mathrm{~F}_{21} \mathrm{O}_{2}^{-}$ & 562.95629 & 562.95734 & 1.9 & PFUnA \\
\hline 10 & $\# 1$ & Perfluorocarboxylates (PFCAs) & PFDoDA & $\mathrm{C}_{12} \mathrm{~F}_{23} \mathrm{O}_{2}^{-}$ & 612.95310 & 612.95386 & 1.2 & $\overline{P F D o D A}$ \\
\hline 11 & $\# 1$ & Perfluorocarboxylates (PFCAs) & PFTrDA & $\mathrm{C}_{13} \mathrm{~F}_{25} \mathrm{O}_{2}^{-}$ & 662.94991 & 662.95172 & 2.7 & PFTrDA \\
\hline 12 & $\# 1$ & Perfluorocarboxylates (PFCAs) & PFTeDA & $\mathrm{C}_{14} \mathrm{~F}_{27} \mathrm{O}_{2}^{-}$ & 712.94671 & 712.94836 & 2.3 & PFTeDA \\
\hline 13 & $\# 1$ & Perfluorocarboxylates (PFCAs) & PFPeDA & $\mathrm{C}_{15} \mathrm{~F}_{29} \mathrm{O}_{2}^{-}$ & 762.94352 & 762.94452 & 1.3 & PFTeDA \\
\hline 14 & $\# 1$ & Perfluorocarboxylates (PFCAs) & PFHXDA & $\mathrm{C}_{16} \mathrm{~F}_{31} \mathrm{O}_{2}^{-}$ & 812.94032 & 812.94269 & 2.9 & PFTeDA \\
\hline 15 & $\# 1$ & Perfluorocarboxylates (PFCAs) & PFHpDA & $\mathrm{C}_{17} \mathrm{~F}_{33} \mathrm{O}_{2}^{-}$ & 862.93713 & 862.93683 & $\mid-0.3$ & PFTeDA \\
\hline 16 & $\# 1$ & Perfluorocarboxylates (PFCAs) & PFOcDA & $\mathrm{C}_{18} \mathrm{~F}_{35} \mathrm{O}_{2}^{-}$ & 912.93394 & 912.93579 & 2.0 & PFTeDA \\
\hline 17 & $\# 1$ & Perfluorocarboxylates (PFCAs) & PFNoDA & $\mathrm{C}_{19} \mathrm{~F}_{37} \mathrm{O}_{2}{ }^{-}$ & 962.93074 & 962.92728 & \begin{tabular}{|l|}
-3.6 \\
\end{tabular} & PFTeDA \\
\hline 18 & $\# 2$ & Perfluoroalkane sulfonates (PFSAs) & PFPrS & $\mathrm{C}_{3} \mathrm{~F}_{7} \mathrm{SO}_{3}^{-}$ & 248.94564 & 248.94627 & 2.5 & PFPrS \\
\hline 19 & $\# 2$ & Perfluoroalkane sulfonates (PFSAs) & PFBS & $\mathrm{C}_{4} \mathrm{~F}_{9} \mathrm{SO}_{3}{ }^{\circ}$ & 298.94244 & 298.94363 & 4.0 & PFBS \\
\hline 20 & $\# 2$ & Perfluoroalkane sulfonates (PFSAs) & PFPeS & $\mathrm{C}_{5} \mathrm{~F}_{11} \mathrm{SO}_{3}{ }^{-}$ & 348.93925 & 348.94055 & \begin{tabular}{|l|}
3.7 \\
\end{tabular} & PFPeS \\
\hline 21 & $\# 2$ & Perfluoroalkane sulfonates (PFSAs) & PFHXS & $\mathrm{C}_{6} \mathrm{~F}_{13} \mathrm{SO}_{3}{ }^{-}$ & 398.93606 & 398.93713 & \begin{tabular}{|l|}
2.7 \\
\end{tabular} & PFHxS \\
\hline
\end{tabular}




\begin{tabular}{|c|c|c|c|c|c|c|c|c|}
\hline 22 & |\#2 & Perfluoroalkane sulfonates (PFSAs) & PFHpS & $\mathrm{C}_{7} \mathrm{~F}_{15} \mathrm{SO}_{3}{ }^{-}$ & 448.93286 & 448.93411 & 2.8 & PFHpS \\
\hline 23 & \#2 & Perfluoroalkane sulfonates (PFSAs) & PFOS & $\mathrm{C}_{8} \mathrm{~F}_{17} \mathrm{SO}_{3}{ }^{-}$ & 498.92967 & 498.93033 & 1.3 & PFOS \\
\hline 24 & \#2 & Perfluoroalkane sulfonates (PFSAs) & PFNS & $\mathrm{C}_{9} \mathrm{~F}_{19} \mathrm{SO}_{3}{ }^{\circ}$ & 548.92647 & 548.92743 & 1.7 & PFOS \\
\hline 25 & $\# 2$ & Perfluoroalkane sulfonates (PFSAs) & PFDS & $\mathrm{C}_{10} \mathrm{~F}_{21} \mathrm{SO}_{3}{ }^{\circ}$ & 598.92328 & 598.92432 & 1.7 & PFDS \\
\hline 26 & \#2 & Perfluoroalkane sulfonates (PFSAs) & PFUnS & $\mathrm{C}_{11} \mathrm{~F}_{23} \mathrm{SO}_{3}{ }^{\circ}$ & 648.92009 & 648.92175 & 2.6 & PFDS \\
\hline 27 & \#2 & Perfluoroalkane sulfonates (PFSAs) & PFDoS & $\mathrm{C}_{12} \mathrm{~F}_{25} \mathrm{SO}_{3}{ }^{\circ}$ & 698.91689 & 698.91846 & 2.2 & PFDS \\
\hline 28 & $\# 2$ & Perfluoroalkane sulfonates (PFSAs) & PFTrDS & $\mathrm{C}_{13} \mathrm{~F}_{27} \mathrm{SO}_{3}{ }^{\circ}$ & 748.91370 & 748.91504 & 1.8 & PFDS \\
\hline 29 & \#2 & Perfluoroalkane sulfonates (PFSAs) & PFTeDS & $\mathrm{C}_{14} \mathrm{~F}_{29} \mathrm{SO}_{3}{ }^{\circ}$ & 798.91050 & 798.91241 & 2.4 & PFDS \\
\hline 30 & \#2 & Perfluoroalkane sulfonates (PFSAs) & PFPeDS & $\mathrm{C}_{15} \mathrm{~F}_{31} \mathrm{SO}_{3}{ }^{\circ}$ & 848.90731 & 848.90948 & 2.6 & PFDS \\
\hline 31 & \#2 & Perfluoroalkane sulfonates (PFSAs) & PFHXDS & $\mathrm{C}_{16} \mathrm{~F}_{33} \mathrm{SO}_{3}{ }^{\circ}$ & 898.90412 & 898.90619 & 2.3 & PFDS \\
\hline 32 & \#2 & |Perfluoroalkane sulfonates (PFSAs) & PFHpDS & $\mathrm{C}_{17} \mathrm{~F}_{35} \mathrm{SO}_{3}{ }^{\circ}$ & 948.90092 & 948.90179 & 0.9 & PFDS \\
\hline 33 & $\# 3$ & |Perfluoroalkyl sulfonamides (FASAs) & FPrSA & $\mathrm{C}_{3} \mathrm{~F}_{7} \mathrm{SO}_{2} \mathrm{NH}^{-}$ & 247.96162 & 247.96019 & -5.8 & FBSA \\
\hline 34 & \#3 & Perfluoroalkyl sulfonamides (FASAs) & FBSA & $\mathrm{C}_{4} \mathrm{~F}_{9} \mathrm{SO}_{2} \mathrm{NH}^{-}$ & 297.95843 & 297.95957 & 3.8 & FBSA \\
\hline 35 & $\# 3$ & Perfluoroalkyl sulfonamides (FASAs) & FPeSA & $\mathrm{C}_{5} \mathrm{~F}_{11} \mathrm{SO}_{2} \mathrm{NH}^{-}$ & 347.95523 & 347.95633 & 3.2 & FHxSA \\
\hline 36 & $\# 3$ & Perfluoroalkyl sulfonamides (FASAs) & $\mathrm{FH} \times \mathrm{SA}$ & $\mathrm{C}_{6} \mathrm{~F}_{13} \mathrm{SO}_{2} \mathrm{NH}^{-}$ & 397.95204 & 397.95293 & 2.2 & FHXSA \\
\hline 37 & $\# 3$ & Perfluoroalkyl sulfonamides (FASAs) & FOSA & $\mathrm{C}_{8} \mathrm{~F}_{17} \mathrm{SO}_{2} \mathrm{NH}^{-}$ & 497.94631 & 497.94654 & 0.5 & FOSA \\
\hline 38 & $\# 4$ & $\mathrm{~N}$-methyl FASAs & MeFOSA & $\mathrm{C}_{9} \mathrm{~F}_{17} \mathrm{SO}_{2} \mathrm{NH}_{3}{ }^{*}$ & 511.96130 & 511.96220 & 1.8 & MeFOSA \\
\hline 39 & \#5 & N-ethyl FASAs & EtFOSA & $\mathrm{C}_{10} \mathrm{~F}_{17} \mathrm{SO}_{2} \mathrm{NH}_{5}$ & 525.97695 & 525.97525 & -3.2 & EtFOSA \\
\hline 40 & \#6 & Perfluoroalkyl sulfonamido acetic acids (FASAAs) & FBSAA & $\mathrm{C}_{6} \mathrm{~F}_{9} \mathrm{SO}_{4} \mathrm{NH}_{3}{ }^{-}$ & 355.96391 & 355.96466 & 2.1 & FOSAA \\
\hline 41 & \#6 & Perfluoroalkyl sulfonamido acetic acids (FASAAs) & FPeSAA & $\mathrm{C}_{7} \mathrm{~F}_{11} \mathrm{SO}_{4} \mathrm{NH}_{3}{ }^{-}$ & 405.96071 & 405.96166 & 2.3 & FOSAA \\
\hline 42 & \#6 & Perfluoroalkyl sulfonamido acetic acids (FASAAs) & FHXSAA & $\mathrm{C}_{8} \mathrm{~F}_{13} \mathrm{SO}_{4} \mathrm{NH}_{3}{ }^{\circ}$ & 455.95752 & 455.95807 & 1.2 & FOSAA \\
\hline 43 & \#6 & Perfluoroalkyl sulfonamido acetic acids (FASAAs) & FOSAA & $\mathrm{C}_{10} \mathrm{~F}_{17} \mathrm{SO}_{4} \mathrm{NH}_{3}{ }^{-}$ & 555.95113 & 555.95204 & 1.6 & FOSAA \\
\hline 44 & \#7 & $\mathrm{N}$-methyl FASAAs & MeFBSAA & $\mathrm{C}_{7} \mathrm{~F}_{9} \mathrm{SO}_{4} \mathrm{NH}_{5}{ }^{-}$ & 369.97956 & 369.98059 & 2.8 & MeFOSAA \\
\hline 45 & \#7 & $\mathrm{N}$-methyl FASAAs & MeFPeSAA & $\mathrm{C}_{8} \mathrm{~F}_{11} \mathrm{SO}_{4} \mathrm{NH}_{5}{ }^{\circ}$ & 419.97636 & 419.97763 & 3.0 & MeFOSAA \\
\hline 46 & \#7 & $\mathrm{N}$-methyl FASAAs & MeFHxSAA & $\mathrm{C}_{9} \mathrm{~F}_{13} \mathrm{SO}_{4} \mathrm{NH}_{5}^{-}$ & 469.97317 & 469.97341 & 0.5 & MeFOSAA \\
\hline
\end{tabular}




\begin{tabular}{|c|c|c|c|c|c|c|c|c|}
\hline 47 & $\# 7$ & $\mathrm{~N}$-methyl FASAAs & MeFHpSAA & $\mathrm{C}_{10} \mathrm{~F}_{15} \mathrm{SO}_{4} \mathrm{NH}_{5}{ }^{-}$ & 519.96998 & 519.97132 & 2.6 & MeFOSAA \\
\hline 48 & $\# 7$ & $\mathrm{~N}$-methyl FASAAs & MeFOSAA & $\mathrm{C}_{11} \mathrm{~F}_{17} \mathrm{SO}_{4} \mathrm{NH}_{5}^{-}$ & 569.96678 & 569.96793 & 2.0 & MeFOSAA \\
\hline 49 & $\# 7$ & $\mathrm{~N}$-methyl FASAAs & MeFNSAA & $\mathrm{C}_{12} \mathrm{~F}_{19} \mathrm{SO}_{4} \mathrm{NH}_{5}^{-}$ & 619.96359 & 619.96078 & -4.5 & MeFOSAA \\
\hline 50 & $\# 8$ & N-ethyl FASAAs & EtFBSAA & $\mathrm{C}_{8} \mathrm{~F}_{9} \mathrm{SO}_{4} \mathrm{NH}_{7}$ & 383.99521 & 383.99620 & 2.6 & EtFOSAA \\
\hline 51 & $\# 8$ & N-ethyl FASAAs & EtFHxSAA & $\mathrm{C}_{10} \mathrm{~F}_{13} \mathrm{SO}_{4} \mathrm{NH}_{7}^{-}$ & 483.98882 & 483.98926 & 0.9 & EtFOSAA \\
\hline 52 & $\# 8$ & N-ethyl FASAAs & EtFHpSAA & $\mathrm{C}_{11} \mathrm{~F}_{15} \mathrm{SO}_{4} \mathrm{NH}_{7}^{-}$ & 533.98562 & 533.98663 & 1.9 & EtFOSAA \\
\hline 53 & $\# 8$ & N-ethyl FASAAs & EtFOSAA & $\mathrm{C}_{12} \mathrm{~F}_{17} \mathrm{SO}_{4} \mathrm{NH}_{7}^{-}$ & 583.98243 & 583.98380 & 2.3 & EtFOSAA \\
\hline 54 & $\# 9$ & $\mathrm{X}: 2$ fluorotelomer sulfonates (X:2 FTSA) & 4:2 FTSA & $\mathrm{C}_{6} \mathrm{~F}_{9} \mathrm{H}_{4} \mathrm{SO}_{3}^{-}$ & 326.97374 & 326.97507 & 4.1 & 4:2 FTSA \\
\hline 55 & $\# 9$ & $\mathrm{X}: 2$ fluorotelomer sulfonates (X:2 FTSA) & 6:2 FTSA & $\mathrm{C}_{8} \mathrm{~F}_{13} \mathrm{H}_{4} \mathrm{SO}_{3}^{-}$ & 426.96866 & 426.96836 & -0.7 & 6:2 FTSA \\
\hline 56 & $\# 9$ & $\mathrm{X}: 2$ fluorotelomer sulfonates (X:2 FTSA) & 8:2 FTSA & $\mathrm{C}_{10} \mathrm{~F}_{17} \mathrm{H}_{4} \mathrm{SO}_{3}{ }^{-}$ & 526.96097 & 526.96215 & 2.2 & 8:2 FTSA \\
\hline 57 & $\# 9$ & $\mathrm{X}: 2$ fluorotelomer sulfonates (X:2 FTSA) & 10:2 FTSA & $\mathrm{C}_{12} \mathrm{~F}_{21} \mathrm{H}_{4} \mathrm{SO}_{3}{ }^{-}$ & 626.95458 & 626.95572 & 1.8 & 8:2 FTSA \\
\hline 58 & $\# 9$ & $\mathrm{X}: 2$ fluorotelomer sulfonates (X:2 FTSA) & 12:2 FTSA & $\mathrm{C}_{14} \mathrm{~F}_{25} \mathrm{H}_{4} \mathrm{SO}_{3}{ }^{-}$ & 726.94819 & 726.94971 & 2.1 & $8: 2$ FTSA \\
\hline 59 & $\# 9$ & $\mathrm{X}: 2$ fluorotelomer sulfonates (X:2 FTSA) & 14:2 FTSA & $\mathrm{C}_{16} \mathrm{~F}_{29} \mathrm{H}_{4} \mathrm{SO}_{3}{ }^{-}$ & 826.94180 & 826.94362 & 2.2 & $8: 2$ FTSA \\
\hline 60 & $\# 9$ & $\mathrm{X}: 2$ fluorotelomer sulfonates (X:2 FTSA) & 16:2 FTSA & $\mathrm{C}_{18} \mathrm{~F}_{33} \mathrm{H}_{4} \mathrm{SO}_{3}{ }^{-}$ & 926.93542 & 926.93772 & 2.5 & 8:2 FTSA \\
\hline 61 & $\# 10$ & Hydroxylated FTSA (OH-X:2 FTSA) & OH-6:2 FTSA & $\mathrm{C}_{8} \mathrm{~F}_{13} \mathrm{H}_{4} \mathrm{SO}_{4}^{-}$ & 442.96227 & 442.96366 & 3.1 & $6: 2$ FTSA \\
\hline 62 & $\# 10$ & Hydroxylated FTSA (OH-X:2 FTSA) & $\mathrm{OH}-8: 2$ FTSA & $\mathrm{C}_{10} \mathrm{~F}_{17} \mathrm{H}_{4} \mathrm{SO}_{4}{ }^{-}$ & 542.95588 & 542.95714 & 2.3 & 8:2 FTSA \\
\hline 63 & $\# 10$ & Hydroxylated FTSA (OH-X:2 FTSA) & $\mathrm{OH}-10: 2 \mathrm{FTSA}$ & $\mathrm{C}_{12} \mathrm{~F}_{21} \mathrm{H}_{4} \mathrm{SO}_{4}{ }_{4}^{-}$ & 642.94949 & 642.95112 & 2.5 & 8:2 FTSA \\
\hline 64 & $\# 10$ & Hydroxylated FTSA (OH-X:2 FTSA) & $\mathrm{OH}-12: 2 \mathrm{FTSA}$ & $\mathrm{C}_{14} \mathrm{~F}_{25} \mathrm{H}_{4} \mathrm{SO}_{4}$ & 742.94311 & 742.94708 & 5.3 & 8:2 FTSA \\
\hline 65 & $\# 10$ & Hydroxylated FTSA (OH-X:2 FTSA) & $\mathrm{OH}-14: 2 \mathrm{FTSA}$ & $\mathrm{C}_{16} \mathrm{~F}_{29} \mathrm{H}_{4} \mathrm{SO}_{4}^{-}$ & 842.93672 & 842.93836 & 1.9 & 8:2 FTSA \\
\hline 66 & $\# 11$ & $\mathrm{X}: 2$ fluorotelomer sulfonamide (X:2 FTSA-PrA) & 6:2 FTSA-PrA & $\mathrm{C}_{8} \mathrm{~F}_{13} \mathrm{SO}_{2} \mathrm{NH}_{5}^{-}$ & 425.98334 & 425.98407 & 1.7 & FOSA \\
\hline 67 & $\# 11$ & $\mathrm{X}: 2$ fluorotelomer sulfonamide (X:2 FTSA-PrA) & $8: 2$ FTSA-PrA & $\mathrm{C}_{10} \mathrm{~F}_{17} \mathrm{SO}_{2} \mathrm{NH}_{5}^{-}$ & 525.97695 & 525.97728 & 0.6 & FOSA \\
\hline 68 & $\# 11$ & $\mathrm{X}: 2$ fluorotelomer sulfonamide (X:2 FTSA-PrA) & 10:2 FTSA-PrA & $\mathrm{C}_{12} \mathrm{~F}_{21} \mathrm{SO}_{2} \mathrm{NH}_{5}^{-}$ & 625.97056 & 625.97176 & 1.9 & FOSA \\
\hline 69 & $\# 12$ & $\mathrm{X}: 3$ fluorotelomer carboxylate (X:3 FTCA, also noted as $\mathrm{X}: 3$ acid) & $5: 3$ acid $(5: 3$ FTCA) & $\mathrm{C}_{8} \mathrm{~F}_{11} \mathrm{H}_{4} \mathrm{O}_{2}^{-}$ & 341.00356 & 341.00316 & -1.2 & 5:3 acid \\
\hline 70 & $\# 12$ & $\mathrm{X}: 3$ fluorotelomer carboxylate (X:3 FTCA, also noted as $\mathrm{X}: 3$ acid) & $7: 3$ acid $(7: 3$ FTCA $)$ & $\mathrm{C}_{10} \mathrm{~F}_{15} \mathrm{H}_{4} \mathrm{O}_{2}^{-}$ & 440.99717 & 440.99755 & 0.9 & $7: 3$ acid \\
\hline 71 & $\# 12$ & $\mathrm{X}: 3$ fluorotelomer carboxylate (X:3 FTCA, also noted as $\mathrm{X}: 3$ acid) & 9:3 acid (9:3 FTCA) & $\mathrm{C}_{12} \mathrm{~F}_{19} \mathrm{H}_{4} \mathrm{O}_{2}^{-}$ & 540.99079 & 540.99246 & 3.1 & $7: 3$ acid \\
\hline
\end{tabular}




\begin{tabular}{|c|c|c|c|c|c|c|c|c|}
\hline 72 & $\# 12$ & X:3 fluorotelomer carboxylate (X:3 FTCA, also noted as $X: 3$ acid) & 11:3 acid (11:3 FTCA) & $\mathrm{C}_{14} \mathrm{~F}_{23} \mathrm{H}_{4} \mathrm{O}_{2}{ }^{-}$ & 640.98440 & 640.98670 & 3.6 & $7: 3$ acid \\
\hline 73 & $\# 13$ & X:2 fluorotelomer carboxylate (X:2 FTCA) & 8:2 FTCA & $\mathrm{C}_{10} \mathrm{~F}_{17} \mathrm{H}_{2} \mathrm{O}_{2}^{-}$ & 476.97833 & 476.98121 & 6.0 & PFDA \\
\hline 74 & $\# 13$ & $\mathrm{X}: 2$ fluorotelomer carboxylate (X:2 FTCA) & 10:2 FTCA & $\mathrm{C}_{12} \mathrm{~F}_{21} \mathrm{H}_{2} \mathrm{O}_{2}^{-}$ & 576.97194 & 576.97279 & 1.5 & PFDoDA \\
\hline 75 & $\# 13$ & $\mathrm{X}: 2$ fluorotelomer carboxylate (X:2 FTCA) & 12:2 FTCA & $\mathrm{C}_{14} \mathrm{~F}_{25} \mathrm{H}_{2} \mathrm{O}_{2}^{-}$ & 676.96555 & 676.96749 & 2.9 & PFTrDA \\
\hline 76 & $\# 14$ & $\mathrm{X}: 2$ fluorotelomer unsaturated carboxylate (X:2 FTUCA) & 6:2 FTUCA & $\mathrm{C}_{8} \mathrm{~F}_{12} \mathrm{HO}_{2}^{-}$ & 356.97849 & 356.97841 & -0.2 & PFOA \\
\hline 77 & $\# 14$ & $\mathrm{X}: 2$ fluorotelomer unsaturated carboxylate (X:2 FTUCA) & 8:2 FTUCA & $\mathrm{C}_{10} \mathrm{~F}_{16} \mathrm{HO}_{2}^{-}$ & 456.97210 & 456.97332 & 2.7 & PFDA \\
\hline 78 & $\# 14$ & $\mathrm{X}: 2$ fluorotelomer unsaturated carboxylate (X:2 FTUCA) & 10:2 FTUCA & $\mathrm{C}_{12} \mathrm{~F}_{20} \mathrm{HO}_{2}^{-}$ & 556.96572 & 556.96573 & 0.0 & PFDoDA \\
\hline 79 & $\# 15$ & Sulfinyl analog of fluorotelomer thioether amidosulfonate & 6:2 FTSO-PrAdDiMePrS (6:2 FTSAS-sulfoxide) & $\mathrm{C}_{15} \mathrm{~F}_{13} \mathrm{H}_{17} \mathrm{NS}_{2} \mathrm{O}_{5}$ & 602.03406 & 602.03577 & 2.8 & 6:2 FTSA \\
\hline 80 & $\# 16$ & Sulfonyl analog of fluorotelomer thioether amidosulfonate & 6:2 FTSO2-PrAdDiMeEtS (6:2-FTSAS-sulfone) & $\mathrm{C}_{15} \mathrm{~F}_{13} \mathrm{H}_{17} \mathrm{NS}_{2} \mathrm{O}_{6}$ & 618.02897 & 618.02996 & 1.6 & 6:2 FTSA \\
\hline 81 & $\# 17$ & Polyfluoroalkyl phosphate diester (diPAP) & $6: 2$ diPAP & $\mathrm{C}_{16} \mathrm{~F}_{26} \mathrm{H}_{8} \mathrm{PO}_{4}^{-}$ & 788.97450 & 788.97622 & 2.2 & 6:2 diPAP \\
\hline 82 & $\# 18$ & $\mathrm{X}: 3$ ketone fluorotelomer thia hydroxy propanoic acids & 7:3 keto-FTTh-OH-PrAcid & $\mathrm{C}_{13} \mathrm{~F}_{15} \mathrm{H}_{6} \mathrm{SO}_{5}{ }^{-}$ & 558.96964 & 558.96674 & -5.2 & PFOA \\
\hline 83 & $\# 18$ & $\mathrm{x}: 3$ ketone fluorotelomer thia hydroxy propanoic acids & 9:3 keto-FTTh-OH-PrAcid & $\mathrm{C}_{15} \mathrm{~F}_{19} \mathrm{H}_{6} \mathrm{SO}_{5}{ }^{\circ}$ & 658.96325 & 658.96049 & -4.2 & PFOA \\
\hline 84 & $\# 19$ & Perfluoroalkyl sulfinates & $\mathrm{PFHxSi}$ & $\mathrm{C}_{6} \mathrm{~F}_{13} \mathrm{SO}_{2}^{-}$ & 382.94114 & 382.94246 & 3.4 & PFHXS \\
\hline 85 & $\# 19$ & Perfluoroalkyl sulfinates & $\mathrm{PFHpSi}$ & $\mathrm{C}_{7} \mathrm{~F}_{15} \mathrm{SO}_{2}^{-}$ & 432.93795 & 432.93922 & 2.9 & PFHpS \\
\hline 86 & $\# 19$ & Perfluoroalkyl sulfinates & PFOSi & $\mathrm{C}_{8} \mathrm{~F}_{17} \mathrm{SO}_{2}^{-}$ & 482.93475 & 482.93580 & 2.2 & PFOS \\
\hline 87 & $\# 19$ & Perfluoroalkyl sulfinates & PFDSi & $\mathrm{C}_{10} \mathrm{~F}_{21} \mathrm{SO}_{2}{ }^{-}$ & 582.92837 & 582.92952 & 2.0 & PFDS \\
\hline 88 & $\# 20$ & Hydrido-PFCAs & H-PFNA & $\mathrm{C}_{9} \mathrm{~F}_{16} \mathrm{HO}_{2}{ }^{-}$ & 444.97210 & 444.97418 & 4.7 & PFNA \\
\hline 89 & $\# 20$ & Hydrido-PFCAs & H-PFUnA & $\mathrm{C}_{11} \mathrm{~F}_{20} \mathrm{HO}_{2}^{-}$ & 544.96571 & 544.96962 & 7.2 & PFUnA \\
\hline 90 & $\# 20$ & Hydrido-PFCAs & H-PFDoA & $\mathrm{C}_{12} \mathrm{~F}_{22} \mathrm{HO}_{2}^{-}$ & 594.96252 & 594.96490 & 4.0 & PFDoDA \\
\hline 91 & $\# 20$ & Hydrido-PFCAs & H-PFTrDA & $\mathrm{C}_{13} \mathrm{~F}_{24} \mathrm{HO}_{2}^{-}$ & 644.95933 & 644.96050 & 1.8 & PFTrDA \\
\hline 92 & $\# 20$ & Hydrido-PFCAs & H-PFTeDA & $\mathrm{C}_{14} \mathrm{~F}_{26} \mathrm{HO}_{2}^{-}$ & 694.95613 & 694.95643 & 0.4 & PFTeDA \\
\hline 93 & \#20 & Hydrido-PFCAs & H-PFPeDA & $\mathrm{C}_{15} \mathrm{~F}_{28} \mathrm{HO}_{2}^{-}$ & 744.95294 & 744.95667 & 5.0 & PFTeDA \\
\hline 94 & $\# 20$ & Hydrido-PFCAs & H-PFHxDA & $\mathrm{C}_{16} \mathrm{~F}_{30} \mathrm{HO}_{2}^{-}$ & 794.94975 & 794.95227 & 3.2 & PFTeDA \\
\hline 95 & \#20 & Hydrido-PFCAs & H-PFHpDA & $\mathrm{C}_{17} \mathrm{~F}_{32} \mathrm{HO}_{2}^{-}$ & 844.94655 & 844.94904 & 2.9 & PFTeDA \\
\hline 96 & $\# 20$ & Hydrido-PFCAs & H-PFOcDA & $\mathrm{C}_{18} \mathrm{~F}_{34} \mathrm{HO}_{2}^{-}$ & 894.94336 & 894.94678 & 3.8 & PFTeDA \\
\hline
\end{tabular}




\begin{tabular}{|c|c|c|c|c|c|c|c|c|}
\hline 97 & $\# 20$ & Hydrido-PFCAs & H-PFNoDA & $\mathrm{C}_{19} \mathrm{~F}_{36} \mathrm{HO}_{2}^{-}$ & 944.94016 & 944.94061 & 0.5 & PFTeDA \\
\hline 98 & \#21 & Hydrido-PFSAs & H-PFEiDA & $\mathrm{C}_{20} \mathrm{~F}_{38} \mathrm{HO}_{2}^{-}$ & 994.93697 & 994.93667 & -0.3 & PFTeDA \\
\hline 99 & \#21 & Hydrido-PFSAs & H-PFHXS & $\mathrm{C}_{6} \mathrm{~F}_{12} \mathrm{HSO}_{3}{ }^{-}$ & 380.94548 & 380.94747 & 5.2 & PFHXS \\
\hline 100 & \#21 & Hydrido-PFSAs & H-PFHpS & $\mathrm{C}_{7} \mathrm{~F}_{14} \mathrm{HSO}_{3}{ }^{-}$ & 430.94228 & 430.94334 & 2.5 & PFHPS \\
\hline 101 & $\# 21$ & Hydrido-PFSAs & H-PFOS & $\mathrm{C}_{8} \mathrm{~F}_{16} \mathrm{HSO}_{3}^{-}$ & 480.93909 & 480.94037 & 2.7 & PFOS \\
\hline 102 & $\# 21$ & Hydrido-PFSAs & H-PFNS & $\mathrm{C}_{9} \mathrm{~F}_{18} \mathrm{HSO}_{3}{ }^{-}$ & 530.93590 & 530.93720 & 2.4 & PFOS \\
\hline 103 & $\# 22$ & Unsaturated PFSAs (U-PFSAs) & 3-UPFSA & $\mathrm{C}_{6} \mathrm{~F}_{11} \mathrm{SO}_{3}{ }^{-}$ & 360.93925 & 360.93852 & -2.0 & PFPrS \\
\hline 104 & $\# 22$ & Unsaturated PFSAs (U-PFSAs) & 4-UPFSA & $\mathrm{C}_{7} \mathrm{~F}_{13} \mathrm{SO}_{3}^{-}$ & 410.93605 & 410.93552 & -1.3 & PFBS \\
\hline 105 & $\# 22$ & Unsaturated PFSAs (U-PFSAs) & 8-UPFSA & $\mathrm{C}_{11} \mathrm{~F}_{21} \mathrm{SO}_{3}{ }^{-}$ & 610.92328 & 610.92453 & 2.0 & PFOS \\
\hline 106 & $\# 22$ & Unsaturated PFSAs (U-PFSAs) & 9-UPFSA & $\mathrm{C}_{12} \mathrm{~F}_{23} \mathrm{SO}_{3}{ }^{-}$ & 660.92009 & 660.92173 & 2.5 & PFOS \\
\hline 107 & \#22 & Unsaturated PFSAs (U-PFSAs) & 10-UPFSA & $\mathrm{C}_{13} \mathrm{~F}_{25} \mathrm{SO}_{3}{ }^{-}$ & 710.91689 & 710.91843 & 2.2 & PFDS \\
\hline 108 & $\# 22$ & Unsaturated PFSAs (U-PFSAs) & 11-UPFSA & $\mathrm{C}_{14} \mathrm{~F}_{27} \mathrm{SO}_{3}{ }^{-}$ & 760.91370 & 760.91559 & 2.5 & PFDS \\
\hline 109 & $\# 22$ & Unsaturated PFSAs (U-PFSAs) & 12-UPFSA & $\mathrm{C}_{15} \mathrm{~F}_{29} \mathrm{SO}_{3}{ }^{\circ}$ & 810.91050 & 810.91192 & 1.8 & PFDS \\
\hline 110 & $\# 22$ & Unsaturated PFSAs (U-PFSAs) & 13-UPFSA & $\mathrm{C}_{16} \mathrm{~F}_{31} \mathrm{SO}_{3}{ }^{-}$ & 860.90731 & 860.91002 & 3.1 & PFDS \\
\hline 111 & $\# 22$ & Unsaturated PFSAs (U-PFSAs) & 14-UPFSA & $\mathrm{C}_{17} \mathrm{~F}_{33} \mathrm{SO}_{3}{ }^{-}$ & 910.90412 & 910.90324 & -1.0 & PFDS \\
\hline 112 & $\# 23$ & Hydrido-U-PFSAs & H-U-PFOS & $\mathrm{C}_{8} \mathrm{~F}_{14} \mathrm{HSO}_{3}{ }^{-}$ & 442.94228 & 442.94490 & 5.9 & PFOS \\
\hline 113 & $\# 23$ & Hydrido-U-PFSAs & H-U-PFDS & $\mathrm{C}_{10} \mathrm{~F}_{18} \mathrm{HSO}_{3}$ & 542.93590 & 542.93106 & -8.9 & PFDS \\
\hline 114 & $\# 24$ & Pentafluorosulfide PFSAs & 8n-F5S-PFSA (F5S-PFOS) & $\mathrm{C}_{8} \mathrm{~F}_{21} \mathrm{~S}_{2} \mathrm{O}_{3}{ }^{-}$ & 606.89535 & 606.89639 & 1.7 & PFOS \\
\hline 115 & \#24 & Pentafluorosulfide PFSAs & 9n-F5S-PFSA (F5S-PFNS) & $\mathrm{C}_{9} \mathrm{~F}_{23} \mathrm{~S}_{2} \mathrm{O}_{3}{ }^{-}$ & 656.89216 & 656.89374 & 2.4 & PFOS \\
\hline 116 & \#24 & Pentafluorosulfide PFSAs & 10n-F5S-PFSA (F5S-PFDS) & $\mathrm{C}_{10} \mathrm{~F}_{25} \mathrm{~S}_{2} \mathrm{O}_{3}{ }^{-}$ & 706.88896 & 706.89056 & 2.3 & PFOS \\
\hline 117 & $\# 25$ & Bisperfluoroalkyl phosphinic acids (X:X PhiA) & 6:6 PhiA & $\mathrm{C}_{12} \mathrm{~F}_{26} \mathrm{PO}_{2}^{-}$ & 700.92207 & 700.92374 & 2.4 & 6:2 diPAP \\
\hline 118 & \#25 & Bisperfluoroalkyl phosphinic acids (X:X PhiA) & 6:8 PhiA & $\mathrm{C}_{14} \mathrm{~F}_{30} \mathrm{PO}_{2}^{-}$ & 800.91568 & 800.91709 & 1.8 & 6:2 diPAP \\
\hline 119 & $\# 25$ & Bisperfluoroalkyl phosphinic acids (X:X PhiA) & 8:8 PhiA & $\mathrm{C}_{16} \mathrm{~F}_{34} \mathrm{PO}_{2}^{-}$ & 900.90929 & 900.91191 & 2.9 & 6:2 diPAP \\
\hline 120 & \#26 & Oxa-PFSA (or PFSA sulfate?) * & O-PFOS & $\mathrm{C}_{8} \mathrm{~F}_{17} \mathrm{SO}_{4}{ }^{-}$ & 514.92458 & 514.92514 & 1.1 & PFOS \\
\hline 121 & \#26 & Oxa-PFSA (or PFSA sulfate?) * & O-PFUnS & $\mathrm{C}_{11} \mathrm{~F}_{23} \mathrm{SO}_{4}^{-}$ & 664.91500 & 664.91613 & 1.7 & PFDS \\
\hline
\end{tabular}




\begin{tabular}{|c|c|c|c|c|c|c|c|c|}
\hline 122 & \#26 & | oxa-PFSA (or PFSA sulfate?) * & O-PFDoS & $\mathrm{C}_{12} \mathrm{~F}_{25} \mathrm{SO}_{4}$ & 714.91181 & 714.91327 & 2.0 & PFDS \\
\hline 123 & $\# 26$ & Oxa-PFSA (or PFSA sulfate?) * & O-PFTrDS & $\mathrm{C}_{13} \mathrm{~F}_{27} \mathrm{SO}_{4}{ }^{-}$ & 764.90861 & 764.91011 & 2.0 & $\begin{array}{l}\text { PFDS } \\
\end{array}$ \\
\hline 124 & \#26 & Oxa-PFSA (or PFSA sulfate?) * & O-PFTeDS & $\mathrm{C}_{14} \mathrm{~F}_{29} \mathrm{SO}_{4}{ }^{-}$ & 814.90542 & 814.90772 & 2.8 & PFDS \\
\hline 125 & $\# 26$ & Oxa-PFSA (or PFSA sulfate?) * & O-PFPeDS & $\mathrm{C}_{15} \mathrm{~F}_{31} \mathrm{SO}_{4}{ }^{-}$ & 864.90222 & 864.90383 & 1.9 & PFDS \\
\hline 126 & $\# 26$ & Oxa-PFSA (or PFSA sulfate?) * & O-PFHxDS & $\mathrm{C}_{16} \mathrm{~F}_{33} \mathrm{SO}_{4}{ }^{-}$ & 914.89903 & 914.90191 & 3.1 & PFDS \\
\hline 127 & \#27 & Oxa-PFCA & PFECA-2 & $\mathrm{C}_{6} \mathrm{~F}_{11} \mathrm{O}_{3}{ }^{\circ}$ & 328.96717 & 328.96617 & -3.0 & PFHXA \\
\hline 128 & \#27 & Oxa-PFCA & PFECA-4 & $\mathrm{C}_{8} \mathrm{~F}_{15} \mathrm{O}_{3}{ }^{-}$ & 428.96079 & 428.96312 & 5.4 & PFHXA \\
\hline 129 & \#28 & Hydrido Oxa-U-PFCA & H-O-U-4-PFCA & $\mathrm{C}_{8} \mathrm{~F}_{10} \mathrm{H}_{3} \mathrm{O}_{3}{ }^{-}$ & 336.99225 & 336.99499 & 8.1 & PFHXA \\
\hline 130 & \#28 & Hydrido Oxa-U-PFCA & H-O-U-5-PFCA & $\mathrm{C}_{9} \mathrm{~F}_{12} \mathrm{H}_{3} \mathrm{O}_{3}{ }^{-}$ & 386.98906 & 386.98630 & -7.1 & PFHXA \\
\hline 131 & $\# 29$ & Chlorinated PFSA & Cl-PFOS & $\mathrm{C}_{8} \mathrm{~F}_{16} \mathrm{ClSO}_{3}{ }^{-}$ & 514.90012 & 514.89988 & -0.5 & PFOS \\
\hline 132 & $\# 30$ & Ketone-PFSA & 10-Keto-PFSA & $\mathrm{C}_{12} \mathrm{~F}_{23} \mathrm{SO}_{4}{ }^{\circ}$ & 676.91500 & 676.91647 & 2.2 & PFDS \\
\hline 133 & $\# 30$ & Ketone-PFSA & 11-Keto-PFSA & $\mathrm{C}_{13} \mathrm{~F}_{25} \mathrm{SO}_{4}{ }^{\circ}$ & 726.91181 & 726.91295 & 1.6 & PFDS \\
\hline 134 & $\# 30$ & Ketone-PFSA & 12-Keto-PFSA & $\mathrm{C}_{14} \mathrm{~F}_{27} \mathrm{SO}_{4}{ }^{\circ}$ & 776.90861 & 776.91012 & 1.9 & PFDS \\
\hline 135 & $\# 30$ & Ketone-PFSA & 13-Keto-PFSA & $\mathrm{C}_{15} \mathrm{~F}_{29} \mathrm{SO}_{4}{ }^{-}$ & 826.90542 & 826.90673 & 1.6 & PFDS \\
\hline 136 & $\# 30$ & Ketone-PFSA & 14-Keto-PFSA & $\mathrm{C}_{16} \mathrm{~F}_{31} \mathrm{SO}_{4}$ & 876.90222 & 876.90475 & 2.9 & PFDS \\
\hline 137 & $\# 30$ & Ketone-PFSA & 15-Keto-PFSA & $\mathrm{C}_{17} \mathrm{~F}_{33} \mathrm{SO}_{4}{ }^{-1}$ & 926.89903 & 926.90318 & 4.5 & PFDS \\
\hline 138 & \#31 & Cyclic perfluoroalkane sulfonate & PFECHS & $\mathrm{C}_{8} \mathrm{~F}_{15} \mathrm{SO}_{3}{ }^{-}$ & 460.93286 & 460.93420 & 2.9 & PFECHS \\
\hline 139 & $\# 32$ & Perfluoroalkyl aldehyde & PFHXAl & $\mathrm{C}_{6} \mathrm{~F}_{11} \mathrm{O}$ & 296.97735 & 296.97828 & 3.1 & PFHXA \\
\hline 140 & $\# 33$ & Perfluoroalkyl oxirane & perfluorotridecane oxirane & $\mathrm{C}_{16} \mathrm{~F}_{22} \mathrm{H}_{4} \mathrm{O}^{-}$ & 724.98310 & 724.98847 & 7.4 & PFTeDA \\
\hline 141 & $\# 33$ & Perfluoroalkyl oxirane & perfluoropentadecane oxirane & $\mathrm{C}_{18} \mathrm{~F}_{3} \mathrm{H}_{4} \mathrm{O}$ & 824.97671 & 824.98128 & 5.5 & PFTeDA \\
\hline 142 & $\# 34$ & Phosphate derivatives & Diammonium-monoPAP-1 & $\mathrm{C}_{17} \mathrm{~F}_{29} \mathrm{H}_{13} \mathrm{~N}_{2} \mathrm{PO}_{5}$ & 907.00990 & 907.01515 & 5.8 & 6:2 diPAP \\
\hline 143 & $\# 34$ & Phosphate derivatives & Diammonium-monoPAP-2 & $\mathrm{C}_{18} \mathrm{~F}_{31} \mathrm{H}_{13} \mathrm{~N}_{2} \mathrm{PO}_{5}$ & 957.00670 & 957.01096 & 4.5 & 6:2 diPAP \\
\hline 144 & $\# 35$ & Trimethylammoniopropyl perfluoroalkyl sulfonamides & TAmPr-FHxSA (PFHxSAmS) & $\mathrm{C}_{12} \mathrm{~F}_{13} \mathrm{H}_{16} \mathrm{~N}_{2} \mathrm{O}_{2} \mathrm{~S}^{+}$ & 499.07249 & 499.07106 & -2.9 & TAmPr-FHxSA \\
\hline 145 & $\# 35$ & Trimethylammoniopropyl perfluoroalkyl sulfonamides & TAmPr-FOSA (PFOSAmS) & $\mathrm{C}_{14} \mathrm{~F}_{17} \mathrm{H}_{16} \mathrm{~N}_{2} \mathrm{O}_{2} \mathrm{~S}^{+}$ & 599.06610 & 599.06477 & -2.2 & TAMPr-FOSA \\
\hline 146 & $\# 36$ & Dimethylammoniopropyl perfluoroalkyl sulfonamides & AmPr-FHxSA (PFHxSAm) & $\mathrm{C}_{11} \mathrm{~F}_{13} \mathrm{H}_{14} \mathrm{~N}_{2} \mathrm{O}_{2} \mathrm{~S}^{+}$ & 485.05684 & 485.05664 & -0.4 & AmPr-FHxSA \\
\hline
\end{tabular}




\begin{tabular}{|c|c|c|c|c|c|c|c|c|}
\hline 147 & \#36 & Dimethylammoniopropyl perfluoroalkyl sulfonamides & AmPr-FOSA (PFOSAm) & $\left|\mathrm{C}_{13} \mathrm{~F}_{17} \mathrm{H}_{14} \mathrm{~N}_{2} \mathrm{O}_{2} \mathrm{~S}^{+}\right|$ & 585.05045 & 585.04951 & -1.6 & AmPr-FOSA \\
\hline 148 & \#37 & Perfluoroalkyl sulfonamidopropyl betaines & CMeAmPr-FOSA (PFOSB) & $\mathrm{C}_{15} \mathrm{~F}_{17} \mathrm{H}_{16} \mathrm{~N}_{2} \mathrm{O}_{4} \mathrm{~S}^{+}$ & 643.05593 & 643.05447 & -2.3 & CMeAmPr-FOSA \\
\hline 149 & \#38 & Perfluoroalkyl sulfonamido N-propylsulfonate, $\mathrm{N}$-propylammonio hydroxyethy & EtOH-AmPr-FOSAPrS (N-HOEAmP-FOSAPS) & $\mathrm{C}_{18} \mathrm{~F}_{17} \mathrm{H}_{24} \mathrm{~N}_{2} \mathrm{O}_{6} \mathrm{~S}_{2}^{+}$ & 751.08043 & 751.07914 & -1.7 & CMeAmPr-FOSA \\
\hline 150 & $\# 39$ & N-sulfopropyl dimethylammoniopropyl perfluoroalkyl sulfonamides & SPAmPr-FHxSA (N-SPAmP-FHxSA) & $\mathrm{C}_{14} \mathrm{~F}_{13} \mathrm{H}_{20} \mathrm{~N}_{2} \mathrm{O}_{5} \mathrm{~S}_{2}^{+}$ & 607.06061 & 607.06212 & 2.5 & TAmPr-FHxSA \\
\hline 151 & $\# 40$ & Perfluoroalkyl amidopropyl amine oxide & OAmPr-FOAd (PFOANO) & $\mathrm{C}_{13} \mathrm{~F}_{15} \mathrm{H}_{14} \mathrm{~N}_{2} \mathrm{O}_{2}^{+}$ & 515.08157 & 515.08216 & 1.1 & OAmPr-FOAd \\
\hline 152 & $\# 41$ & Fluorotelomer sulfonamidopropyl betaines (X:2 FTSA-PrB) & 4:2 FTSA-PrB (4:2 FTAB) & $\mathrm{C}_{13} \mathrm{~F}_{9} \mathrm{H}_{20} \mathrm{~N}_{2} \mathrm{O}_{4} \mathrm{~S}^{+}$ & 471.10001 & 471.10016 & 0.3 & 6:2 FTSA-PrB (6:2 FTAB) \\
\hline 153 & $\# 41$ & Fluorotelomer sulfonamidopropyl betaines (X:2 FTSA-PrB) & 6:2 FTSA-PrB (6:2 FTAB) & $\mathrm{C}_{15} \mathrm{~F}_{13} \mathrm{H}_{20} \mathrm{~N}_{2} \mathrm{O}_{4} \mathrm{~S}^{+}$ & 571.09362 & 571.09353 & -0.2 & 6:2 FTSA-PrB (6:2 FTAB) \\
\hline 154 & \#41 & Fluorotelomer sulfonamidopropyl betaines (X:2 FTSA-PrB) & 8:2 FTSA-PrB (8:2 FTAB) & $\mathrm{C}_{17} \mathrm{~F}_{17} \mathrm{H}_{2} \mathrm{~N}_{2} \mathrm{O}_{4} \mathrm{~S}^{+}$ & 671.08723 & 671.08584 & -2.1 & 6:2 FTSA-PrB (6:2 FTAB) \\
\hline 155 & $\# 41$ & Fluorotelomer sulfonamidopropyl betaines (X:2 FTSA-PrB) & 10:2 FTSA-PrB (10:2 FTAB) & $\mathrm{C}_{19} \mathrm{~F}_{21} \mathrm{H}_{20} \mathrm{~N}_{2} \mathrm{O}_{4} \mathrm{~S}^{+}$ & 771.08084 & 771.08078 & -0.1 & 6:2 FTSA-PrB (6:2 FTAB) \\
\hline 156 & $\# 41$ & Fluorotelomer sulfonamidopropyl betaines (X:2 FTSA-PrB) & 12:2 FTSA-PrB (12:2 FTAB) & $\mathrm{C}_{21} \mathrm{~F}_{25} \mathrm{H}_{20} \mathrm{~N}_{2} \mathrm{O}_{4} \mathrm{~S}^{+}$ & 871.07446 & 871.07122 & -3.7 & 6:2 FTSA-PrB (6:2 FTAB) \\
\hline 157 & $\# 41$ & Fluorotelomer sulfonamidopropyl betaines (X:2 FTSA-PrB) & 14:2 FTSA-PrB (14:2 FTAB) & $\mathrm{C}_{23} \mathrm{~F}_{29} \mathrm{H}_{20} \mathrm{~N}_{2} \mathrm{O}_{4} \mathrm{~S}^{+}$ & 971.06807 & 971.06611 & -2.0 & 6:2 FTSA-PrB (6:2 FTAB) \\
\hline 158 & $\# 42$ & Fluorotelomer sulfonamidopropyl dimethylamines (X:2 FTSA-PrDiMeAn) & 6:2 FTSA-PrDiMeAn & $\mathrm{C}_{13} \mathrm{~F}_{13} \mathrm{H}_{18} \mathrm{~N}_{2} \mathrm{O}_{2} \mathrm{~S}^{+}$ & 513.08814 & 513.08763 & -1.0 & AmPr-FHxSA \\
\hline 159 & $\# 42$ & Fluorotelomer sulfonamidopropyl dimethylamines (X:2 FTSA-PrDiMeAn) & 8:2 FTSA-PrDiMeAn & $\mathrm{C}_{15} \mathrm{~F}_{17} \mathrm{H}_{18} \mathrm{~N}_{2} \mathrm{O}_{2} \mathrm{~S}^{+}$ & 613.08175 & 613.08106 & -1.1 & AmPr-FHxSA \\
\hline 160 & $\# 42$ & Fluorotelomer sulfonamidopropyl dimethylamines (X:2 FTSA-PrDiMeAn) & 10:2 FTSA-PrDiMeAn & $\mathrm{C}_{17} \mathrm{~F}_{21} \mathrm{H}_{18} \mathrm{~N}_{2} \mathrm{O}_{2} \mathrm{~S}^{+}$ & 713.07536 & 713.07094 & -6.2 & AmPr-FHxSA \\
\hline
\end{tabular}

*The low concentration of Oxa-PFSA did not allow for a reliable MS/MS spectrum to be generated for confirmation. 
Table S14. Overall detection frequencies (\%) and concentration ranges ( $\mu \mathrm{g} / \mathrm{kg}$ in samples with PFAS detection) of the 160 detected homologs in OWP samples $(\mathrm{N}=47)$.

\begin{tabular}{|c|c|c|c|}
\hline & $\begin{array}{c}\text { Detection frequency } \\
\%\end{array}$ & $\underset{\mu g / k g}{\operatorname{Min}}$ & $\begin{array}{l}\operatorname{Max} \\
\mu \mathrm{g} / \mathrm{kg}\end{array}$ \\
\hline PFPrA & 6.4 & 0.22 & 1.75 \\
\hline PFBA & 51.1 & 0.10 & 3.74 \\
\hline PFPeA & 25.5 & 0.06 & 2.96 \\
\hline PFHxA & 44.7 & 0.05 & 32.0 \\
\hline PFHpA & 27.7 & 0.21 & 3.41 \\
\hline PFOA & 68.1 & 0.03 & 20.3 \\
\hline PFNA & 27.7 & 0.13 & 5.12 \\
\hline PFDA & 36.2 & 0.04 & 15.80 \\
\hline PFUnA & 21.3 & 0.13 & 22.3 \\
\hline PFDoDA & 34.0 & 0.02 & 7.84 \\
\hline PFTrDA & 27.7 & 0.01 & 3.20 \\
\hline PFTeDA & 42.6 & 0.004 & 2.72 \\
\hline PFPeDA & 12.8 & 0.07 & 0.71 \\
\hline PFHxDA & 23.4 & 0.05 & 0.80 \\
\hline PFHpDA & 25.5 & 0.03 & 1.00 \\
\hline PFOcDA & 17.0 & 0.01 & 0.42 \\
\hline PFNoDA & 6.4 & 0.02 & 0.24 \\
\hline PFPrS & 4.3 & 0.05 & 0.19 \\
\hline PFBS & 44.7 & 0.01 & 2.62 \\
\hline PFPeS & 10.6 & 0.01 & 0.50 \\
\hline PFHxS & 59.6 & 0.01 & 7.29 \\
\hline PFHpS & 21.3 & 0.01 & 2.29 \\
\hline PFOS & 72.3 & 0.02 & 284 \\
\hline PFNS & 12.8 & 0.01 & 1.90 \\
\hline PFDS & 12.8 & 0.08 & 6.83 \\
\hline PFUnS & 8.5 & 0.02 & 1.64 \\
\hline PFDos & 4.3 & 0.04 & 4.27 \\
\hline PFTrDS & 12.8 & 0.05 & 8.27 \\
\hline PFTeDS & 10.6 & 0.07 & 5.52 \\
\hline PFPeDS & 8.5 & 0.07 & 1.27 \\
\hline PFHxDS & 10.6 & 0.05 & 2.93 \\
\hline PFHpDS & 2.1 & 0.03 & 0.03 \\
\hline FPrSA & 19.1 & 0.03 & 8.59 \\
\hline FBSA & 10.6 & 0.04 & 0.21 \\
\hline FPeSA & 4.3 & 0.05 & 0.18 \\
\hline FHxSA & 10.6 & 0.30 & 1.77 \\
\hline FOSA & 17.0 & 0.02 & 5.42 \\
\hline MeFOSA & 4.3 & 0.49 & 1.06 \\
\hline EtFOSA & 4.3 & 0.39 & 1.51 \\
\hline FBSAA & 8.5 & 0.01 & 0.07 \\
\hline FPeSAA & 4.3 & 0.05 & 0.05 \\
\hline FHxSAA & 6.4 & 0.04 & 0.44 \\
\hline FOSAA & 38.3 & 0.11 & 6.30 \\
\hline MeFBSAA & 23.4 & 0.03 & 2.07 \\
\hline MeFPeSAA & 6.4 & 0.17 & 0.33 \\
\hline MeFHxSAA & 10.6 & 0.17 & 1.98 \\
\hline MeFHpSAA & 6.4 & 0.13 & 0.87 \\
\hline MeFOSAA & 31.9 & 0.14 & 42.9 \\
\hline MeFNSAA & 12.8 & 0.23 & 3.53 \\
\hline EtFBSAA & 4.3 & 0.05 & 0.52 \\
\hline EtFHxSAA & 4.3 & 0.18 & 0.44 \\
\hline
\end{tabular}




\begin{tabular}{|c|c|c|c|}
\hline EtFHpSAA & 8.5 & 0.23 & 1.83 \\
\hline EtFOSAA & 51.1 & 0.17 & 582 \\
\hline $4: 2$ FTSA & 4.3 & 0.01 & 0.03 \\
\hline 6:2 FTSA & 44.7 & 0.05 & 18.3 \\
\hline 8:2 FTSA & 36.2 & 0.04 & 10.7 \\
\hline 10:2 FTSA & 27.7 & 0.01 & 14.1 \\
\hline 12:2 FTSA & 23.4 & 0.11 & 4.79 \\
\hline 14:2 FTSA & 27.7 & 0.17 & 10.5 \\
\hline 16:2 FTSA & 14.9 & 0.01 & 2.43 \\
\hline $\mathrm{OH}-6: 2$ FTSA & 2.1 & - & 0.16 \\
\hline OH-8:2 FTSA & 8.5 & 0.05 & 0.20 \\
\hline $\mathrm{OH}-10: 2$ FTSA & 4.3 & 0.05 & 0.17 \\
\hline $\mathrm{OH}-12: 2 \mathrm{FTSA}$ & 8.5 & 0.06 & 6.31 \\
\hline $\mathrm{OH}-14: 2$ FTSA & 6.4 & 0.14 & 2.11 \\
\hline 6:2 FTSA-PrA & 17.0 & 0.11 & 3.08 \\
\hline 8:2 FTSA-PrA & 8.5 & 0.31 & 0.57 \\
\hline 10:2 FTSA-PrA & 12.8 & 0.13 & 0.86 \\
\hline $5: 3$ acid (5:3 FTCA) & 14.9 & 0.39 & 32.6 \\
\hline 7:3 acid (7:3 FTCA) & 23.4 & 0.25 & 55.9 \\
\hline $9: 3$ acid (9:3 FTCA) & 23.4 & 0.74 & 65.7 \\
\hline $11: 3$ acid (11:3 FTCA) & 19.1 & 0.14 & 12.1 \\
\hline 8:2 FTCA & 8.5 & 0.51 & 16.7 \\
\hline 10:2 FTCA & 4.3 & 0.14 & 0.28 \\
\hline 12:2 FTCA & 2.1 & - & 1.12 \\
\hline 6:2 FTUCA & 4.3 & 0.03 & 1.49 \\
\hline 8:2 FTUCA & 8.5 & 0.04 & 0.89 \\
\hline 10:2 FTUCA & 23.4 & 0.04 & 0.93 \\
\hline 6:2 FTSO-PrAdDiMePrS & 4.3 & 0.45 & 0.73 \\
\hline 6:2 FTSO2-PrAdDiMeEtS & 4.3 & 0.22 & 0.33 \\
\hline $6: 2$ diPAP & 34.0 & 0.12 & 7.57 \\
\hline 7:3 keto-FTTh-OH-PrAcid & 6.4 & 0.10 & 10.9 \\
\hline 9:3 keto-FTTh-OH-PrAcid & 2.1 & - & 0.42 \\
\hline $\mathrm{PFH} \times \mathrm{Si}$ & 17.0 & 0.03 & 0.70 \\
\hline PFHpSi & 4.3 & 0.02 & 0.02 \\
\hline PFOSi & 19.1 & 0.12 & 5.17 \\
\hline PFDSi & 4.3 & 0.03 & 0.14 \\
\hline H-PFNA & 6.4 & 0.21 & 0.34 \\
\hline H-PFUnA & 2.1 & 0.53 & 0.53 \\
\hline H-PFDoA & 8.5 & 0.06 & 0.19 \\
\hline H-PFTrDA & 2.1 & - & 0.05 \\
\hline H-PFTeDA & 2.1 & - & 0.04 \\
\hline H-PFPeDA & 12.8 & 0.04 & 0.45 \\
\hline H-PFHxDA & 8.5 & 0.01 & 0.06 \\
\hline H-PFHpDA & 8.5 & 0.02 & 0.05 \\
\hline H-PFOcDA & 6.4 & 0.05 & 0.17 \\
\hline H-PFNoDA & 4.3 & 0.01 & 0.15 \\
\hline H-PFEiDA & 6.4 & 0.01 & 0.18 \\
\hline H-PFHxS & 4.3 & 0.01 & 0.02 \\
\hline H-PFHpS & 2.1 & - & 0.02 \\
\hline H-PFOS & 19.1 & 0.01 & 2.77 \\
\hline H-PFNS & 4.3 & 0.14 & 0.34 \\
\hline 3-UPFSA & 4.3 & 0.08 & 0.14 \\
\hline 4-UPFSA & 8.5 & 0.04 & 0.57 \\
\hline 8-UPFSA & 4.3 & 0.04 & 0.18 \\
\hline 9-UPFSA & 6.4 & 0.17 & 0.76 \\
\hline 10-UPFSA & 10.6 & 0.02 & 1.91 \\
\hline 11-UPFSA & 8.5 & 0.15 & 2.11 \\
\hline 12-UPFSA & 6.4 & 0.11 & 0.79 \\
\hline
\end{tabular}




\begin{tabular}{|c|c|c|c|}
\hline 13-UPFSA & 6.4 & 0.03 & 0.92 \\
\hline 14-UPFSA & 6.4 & 0.04 & 0.21 \\
\hline H-U-PFOS & 4.3 & 0.03 & 0.24 \\
\hline H-U-PFDS & 6.4 & 0.02 & 1.14 \\
\hline 8n-F5S-PFAS & 10.6 & 0.09 & 4.84 \\
\hline 9n-F5S-PFAS & 8.5 & 0.12 & 1.72 \\
\hline 10n-F5S-PFSA & 4.3 & 0.08 & 0.26 \\
\hline 6:6 PhiA & 21.3 & 0.02 & 1.83 \\
\hline 8:6 PhiA & 40.4 & 0.03 & 13.0 \\
\hline 8:8 PhiA & 40.4 & 0.05 & 8.65 \\
\hline O-PFOS & 2.1 & - & 0.07 \\
\hline O-PFUnS & 6.4 & 0.02 & 0.14 \\
\hline O-PFDoS & 4.3 & 0.05 & 0.29 \\
\hline O-PFTrDS & 2.1 & - & 0.25 \\
\hline O-PFTeDS & 8.5 & 0.06 & 1.03 \\
\hline O-PFPeDS & 4.3 & 0.04 & 0.34 \\
\hline O-PFHxDS & 10.6 & 0.02 & 0.82 \\
\hline PFECA-2 & 2.1 & - & 3.40 \\
\hline PFECA-4 & 12.8 & 0.04 & 0.66 \\
\hline H-O-U-4-PFCA & 10.6 & 0.16 & 0.49 \\
\hline H-O-U-5-PFCA & 2.1 & - & 0.31 \\
\hline Cl-PFOS & 6.4 & 0.13 & 1.29 \\
\hline 10-Keto-PFSA & 2.1 & - & 0.38 \\
\hline 11-Keto-PFSA & 2.1 & - & 0.40 \\
\hline 12-Keto-PFSA & 4.3 & 0.31 & 0.55 \\
\hline 13-Keto-PFSA & 2.1 & - & 0.29 \\
\hline 14-Keto-PFSA & 8.5 & 0.06 & 1.28 \\
\hline 15-Keto-PFSA & 2.1 & - & 0.01 \\
\hline PFECHS & 4.3 & 0.06 & 0.12 \\
\hline PFHXAI & 8.5 & 0.07 & 0.30 \\
\hline perfluorotridecane oxirane & 6.4 & 0.39 & 3.62 \\
\hline perfluoropentadecane oxirane & 6.4 & 0.12 & 0.61 \\
\hline Diammonium-monoPAP-1 & 2.1 & - & 0.13 \\
\hline Diammonium-monoPAP-2 & 2.1 & - & 0.04 \\
\hline TAmPr-FHxSA (PFHxSAmS) & 21.3 & 0.07 & 8.32 \\
\hline TAmPr-FOSA (PFOSAmS) & 27.7 & 0.36 & 17.0 \\
\hline AmPr-FHxSA (PFHxSAm) & 14.9 & 0.17 & 5.03 \\
\hline AmPr-FOSA (PFOSAm) & 17.0 & 0.49 & 6.02 \\
\hline CMeAmPr-FOSA (PFOSB) & 6.4 & 1.22 & 8.25 \\
\hline 8N-HOEAmP-FASAPS & 10.6 & 0.24 & 1.23 \\
\hline N-SPAmP-FHxSA & 2.1 & - & 0.02 \\
\hline OAmPr-FOAd (PFOANO) & 6.4 & 0.09 & 2.03 \\
\hline 4:2 FTSA-PrB (4:2 FTAB) & 2.1 & - & 1.50 \\
\hline 6:2 FTSA-PrB (6:2 FTAB) & 44.7 & 0.21 & 1234 \\
\hline 8:2 FTSA-PrB (8:2 FTAB) & 21.3 & 4.70 & 56.1 \\
\hline 10:2 FTSA-PrB (10:2 FTAB) & 27.7 & 1.74 & 40.3 \\
\hline 12:2 FTSA-PrB (12:2 FTAB) & 23.4 & 0.81 & 23.9 \\
\hline 14:2 FTSA-PrB (14:2 FTAB) & 12.8 & 0.46 & 8.01 \\
\hline 6:2 FTSA-PrDiMeAn & 10.6 & 0.60 & 37.3 \\
\hline 8:2 FTSA-PrDiMeAn & 14.9 & 0.07 & 1.97 \\
\hline 10:2 FTSA-PrDiMeAn & 2.1 & - & 0.30 \\
\hline
\end{tabular}


Table S15. PFAS concentrations $(\mu \mathrm{g} / \mathrm{kg})$ for each of the seven PFAS superclasses in the 47 OWP samples (data corresponding to Figures 3a/3c).

\begin{tabular}{|c|c|c|c|c|c|c|c|}
\hline \multirow[b]{2}{*}{ Sample name } & \multicolumn{7}{|c|}{ Concentration $(\mu \mathrm{g} / \mathrm{kg})$} \\
\hline & PFCAs(-) & PFSAs(-) & ECF-sulfonamides(-) & Fluorotelomers(-) & Miscellaneous(-) & $\mathrm{ECF}(+)$ & Fluorotelomers(+) \\
\hline SLU (Couhins, 1976) & 0.08 & 0.35 & 1.94 & 0.00 & 0.00 & 10.31 & 0.00 \\
\hline SLU (Couhins, 1978) & 2.64 & 28.01 & 19.61 & 2.74 & 0.21 & 15.52 & 0.00 \\
\hline SLU (Couhins, 1980) & 2.06 & 9.65 & 2.62 & 0.00 & 0.00 & 20.40 & 0.00 \\
\hline SLU (Couhins, 1985) & 4.37 & 13.70 & 27.31 & 1.67 & 3.29 & 16.46 & 0.21 \\
\hline SLU (Couhins, 1993) & 7.14 & 148.11 & 113.74 & 21.89 & 9.02 & 22.86 & 5.53 \\
\hline SLU (La Bouzule, 1996) & 5.05 & 193.67 & 640.90 & 15.69 & 31.02 & 0.00 & 14.56 \\
\hline SLU (Couhins, 1998) & 4.30 & 44.17 & 22.22 & 16.22 & 1.77 & 16.47 & 2.45 \\
\hline SLU (PROspective, 2009) & 75.00 & 295.33 & 32.62 & 141.82 & 17.21 & 18.93 & 149.71 \\
\hline SLU (PROspective, 2016) & 26.53 & 98.46 & 34.82 & 29.20 & 2.74 & 0.41 & 192.67 \\
\hline SLU (Réunion, 2017) & 2.32 & 27.86 & 8.29 & 5.82 & 0.44 & 0.00 & 294.23 \\
\hline C-GWS (La Bouzule, 1996) & 19.28 & 160.34 & 100.69 & 19.52 & 32.34 & 28.52 & 18.18 \\
\hline C-GWS (PROspective, 2009) & 55.28 & 71.00 & 20.65 & 57.55 & 4.73 & 2.64 & 93.36 \\
\hline C-GWS (QualiAgro, 2011) & 43.11 & 55.67 & 18.03 & 69.24 & 3.99 & 1.88 & 300.43 \\
\hline C-GWS (QualiAgro, 2013) & 21.41 & 29.16 & 12.97 & 58.19 & 5.00 & 2.17 & 190.55 \\
\hline C-GWS (PROspective, 2016) & 93.66 & 70.49 & 19.17 & 199.67 & 3.94 & 1.24 & 246.21 \\
\hline C-GWS Réunion, 2017) & 10.79 & 20.19 & 31.61 & 15.44 & 0.31 & 0.00 & 399.42 \\
\hline C-BIOW (PROspective, 2009) & 9.10 & 1.14 & 3.17 & 24.97 & 0.66 & 0.00 & 5.41 \\
\hline C-BIOW (QualiAgro, 2011) & 5.35 & 0.45 & 0.34 & 3.97 & 0.10 & 0.00 & 1.75 \\
\hline C-BIOW (QualiAgro, 2013) & 0.37 & 0.14 & 0.21 & 0.27 & 0.30 & 0.00 & 0.94 \\
\hline C-BIOW (PROspective, 2016) & 1.33 & 1.90 & 0.24 & 1.18 & 0.72 & 0.00 & 28.81 \\
\hline C-MSW (La Bouzule, 1996) & 38.41 & 55.33 & 93.51 & 27.56 & 7.34 & 0.27 & 2.50 \\
\hline C-MSW (QualiAgro, 2011) & 29.69 & 7.48 & 2.55 & 27.01 & 0.31 & 1.29 & 61.86 \\
\hline C-MSW (QualiAgro, 2013) & 7.29 & 1.16 & 1.27 & 25.77 & 1.09 & 0.60 & 1297.11 \\
\hline DIG-UW (PROspective, 2016) & 0.31 & 0.45 & 0.00 & 0.00 & 0.18 & 0.00 & 10.11 \\
\hline FYM-DC (QualiAgro, 2011) & 0.553 & 0.332 & 0.000 & 0.000 & 0.230 & 0.000 & 0.000 \\
\hline FYM-DC (QualiAgro, 2013) & 0.14 & 0.56 & 0.93 & 0.05 & 0.00 & 0.00 & 0.00 \\
\hline FYM-DC (EFELE, 2014) & 0.35 & 0.03 & 0.00 & 0.00 & 0.00 & 0.00 & 0.00 \\
\hline FYM-DC (PROspective, 2016) & 0.74 & 0.52 & 0.00 & 0.14 & 0.00 & 0.00 & 0.00 \\
\hline FYM-DC (EFELE, 2016) & 0.30 & 0.03 & 0.30 & 0.00 & 0.00 & 0.00 & 0.00 \\
\hline FYM-DC (EFELE, 2018) & 0.59 & 0.00 & 1.53 & 0.00 & 0.00 & 0.00 & 0.00 \\
\hline PS (EFELE, 2014) & 0.00 & 0.04 & 0.00 & 0.00 & 0.00 & 0.00 & 0.00 \\
\hline PS (EFELE, 2016) & 0.00 & 0.00 & 0.00 & 0.00 & 0.00 & 0.00 & 0.00 \\
\hline PS (Réunion, 2017) & 0.00 & 0.00 & 0.00 & 0.00 & 0.00 & 0.00 & 0.00 \\
\hline PS (EFELE, 2018) & 0.00 & 0.00 & 0.00 & 0.00 & 0.00 & 0.00 & 0.00 \\
\hline PM (EFELE, 2014) & 0.42 & 0.00 & 0.00 & 0.00 & 0.00 & 0.00 & 0.00 \\
\hline PM (EFELE, 2016) & 0.28 & 0.00 & 1.62 & 0.00 & 0.00 & 0.00 & 0.00 \\
\hline PM (Réunion, 2017) & 0.14 & 0.01 & 0.00 & 0.00 & 0.17 & 0.00 & 0.00 \\
\hline PM (EFELE, 2018) & 0.72 & 0.00 & 0.54 & 0.00 & 0.00 & 0.00 & 0.00 \\
\hline C-FYM-DC (PROspective, 2016) & 0.00 & 1.29 & 0.00 & 0.10 & 0.00 & 0.00 & 0.00 \\
\hline C-FYM-P (EFELE, 2014) & 0.10 & 0.02 & 0.11 & 0.00 & 0.18 & 0.00 & 0.00 \\
\hline C-FYM-P (EFELE, 2016) & 0.21 & 0.00 & 0.00 & 0.00 & 0.18 & 0.00 & 0.00 \\
\hline C-FYM-P (EFELE, 2018) & 0.37 & 0.05 & 0.24 & 0.00 & 0.37 & 0.00 & 0.00 \\
\hline DIG-PS (EFELE, 2014) & 0.00 & 0.00 & 0.00 & 0.00 & 0.00 & 0.00 & 0.00 \\
\hline DIG-PS (EFELE, 2016) & 0.96 & 0.29 & 0.52 & 0.00 & 0.00 & 0.00 & 0.00 \\
\hline DIG-PS (EFELE, 2018) & 0.72 & 0.03 & 0.00 & 0.00 & 0.00 & 0.00 & 0.00 \\
\hline PSLU (La Bouzule, 1996) & 11.70 & 37.16 & 43.22 & 4.74 & 3.00 & 0.00 & 0.00 \\
\hline ASH (La Bouzule, 1996) & 0.079 & 0.229 & 0.022 & 0.00 & 0.00 & 0.00 & 0.00 \\
\hline
\end{tabular}

SLU: sewage sludge; C-GWS: compost of green wastes and sludge; C-BIOW: compost of biowastes; C-MSW: compost of municipal solid waste; DIG-UW: digestate of urban wastes; FYM-DC: farmyard manure of dairy cattle; PS: pig slurry; PM: poultry manure; C-FYM-DC: compost of farmyard manure of dairy cattle; DIG-PS: digestate of pig slurry; PSLU: paper sludge (mill sludge); ASH: combustion ashes). 
Table S16. The main PFAS classes detected in agricultural OWP screened in the present study $(n=21)$, ranked by maximum observed summed concentration ( $\Sigma$ class). Corresponding superclass (and ESI detection mode), detection rate, and representative homologs are also included.

\begin{tabular}{llccc} 
Class & Superclass & Max. $\sum$ class (rank /42) & Detection rate (rank /42) & Homologs \\
\hline FASAA & ECF sulfonamide(-) & $1.6 \mu \mathrm{g} / \mathrm{kg}(1)$ & $29 \%(3)$ & FOSAA \\
PFSA & PFAAs(-) & $1.3 \mu \mathrm{g} / \mathrm{kg}(2)$ & $57 \%(2)$ & PFOS \\
PFCA & PFAAs(-) & $0.96 \mu \mathrm{g} / \mathrm{kg}(3)$ & $71 \%(1)$ & PFBA, PFOA \\
EtFASAA & ECF sulfonamide(-) & $0.93 \mu \mathrm{g} / \mathrm{kg}(4)$ & $10 \%(6)$ & EtFOSAA \\
H-PFCA & Miscellaneous(-) & $0.37 \mu \mathrm{g} / \mathrm{kg}(5)$ & $19 \%(4)$ & H-PFPeDA \\
X:Y PhiA & Miscellaneous(-) & $0.23 \mu \mathrm{g} / \mathrm{kg} \mathrm{(6)}$ & $5 \%(7)$ & $8: 8$ PhiA \\
X:2 FTSA & Fluorotelomer(-) & $0.14 \mu \mathrm{g} / \mathrm{kg} \mathrm{(7)}$ & $14 \%(5)$ & $6: 2$ FTSA \\
\hline
\end{tabular}




\section{SI Figures}

Figure S1. Principal component analysis (with Spearman correlation matrixes) of chemical properties for the studied organic waste products.

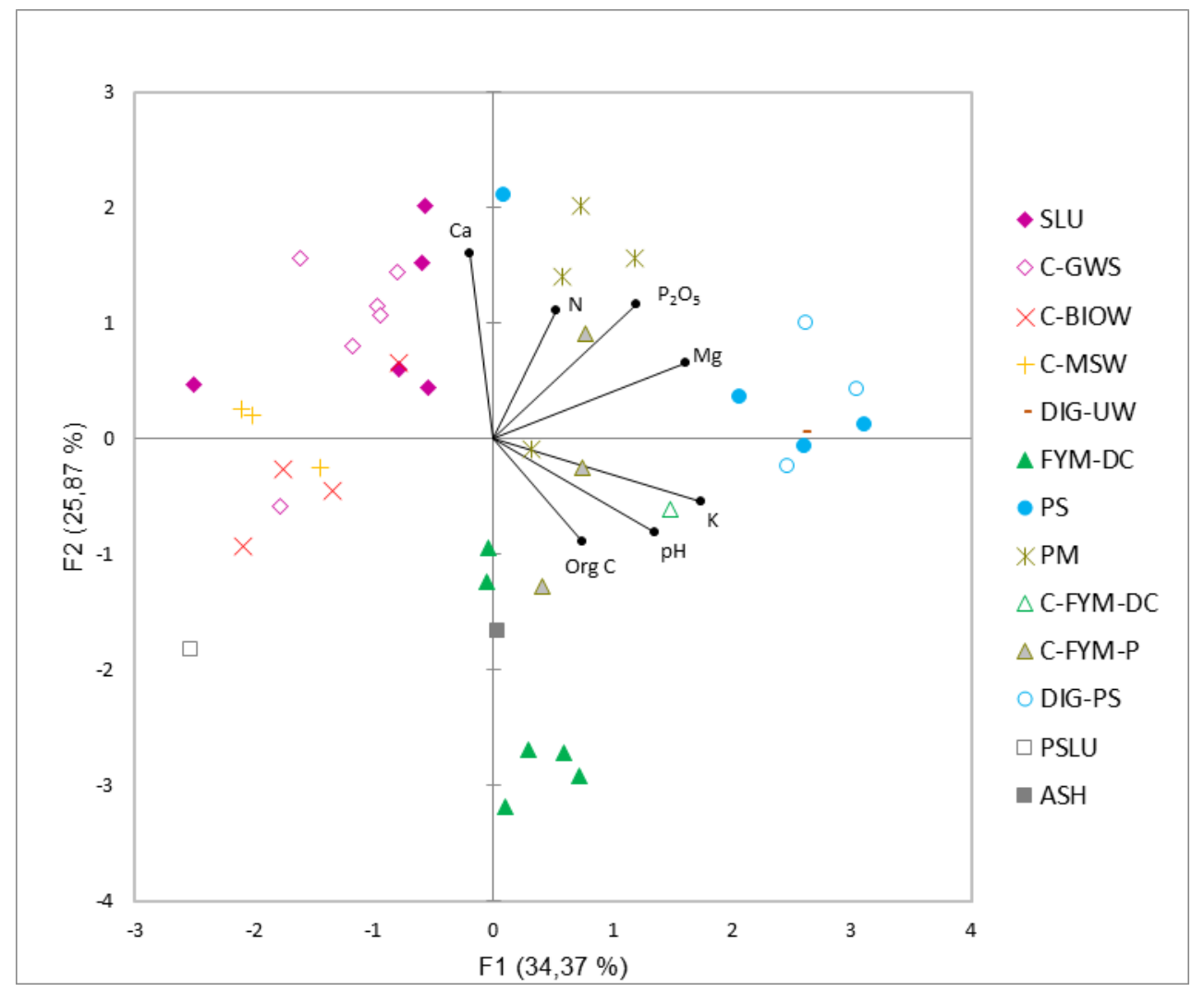


Figure S2. Chromatograms and elucidated high-resolution MS/MS spectra of PFAS detected at high intensity level in urban OWP. When available, a certified reference standard or AFFF material was also analyzed for comparison.

- $E S I(-)$ PFAS

(S2-a). PFOS.

(S2-b). H-PFOS.

(S2-c). PFOSi.

(S2-d). F5S-PFNS.

(S2-e). FHxSAA.

(S2-f). MeFNSAA.

(S2-g). EtFHxSAA.

(S2-h). EtFHpSAA.

(S2-i). EtFOSAA.

(S2-j). 6:2 FTSA-PrA (6:2 fluorotelomer sulfonamide).

(S2-k). 9:3 acid (9:3 FTCA).

(S2-I). 11:3 acid (11:3 FTCA).

(S2-m). 6:2 FTSO-PrAdDiMePrS (6:2 FTSAS-sulfoxide).

(S2-n). 6:2 FTSO2-PrAdDiMeEtS (6:2 FTSAS-sulfone).

(S2-0). 6:6 PhiA.

- $E S I(+)$ PFAS

(S2-p). T-AmPr-FOSA (PFOSAmS).

(S2-q). AmPr-FOSA (PFOSAm).

(S2-r). CMeAmPr-FOSA (PFOSB).

(S2-s). 6:2 FTSA-PrB (6:2 FTAB).

(S2-t). 8:2 FTSA-PrB (8:2 FTAB). 
(S2-a). PFOS, compost of residual municipal solid waste (C-MSW) from La Bouzule site. A certified standard (from Wellington Labs) was also analyzed for comparison.

\section{PFOS}
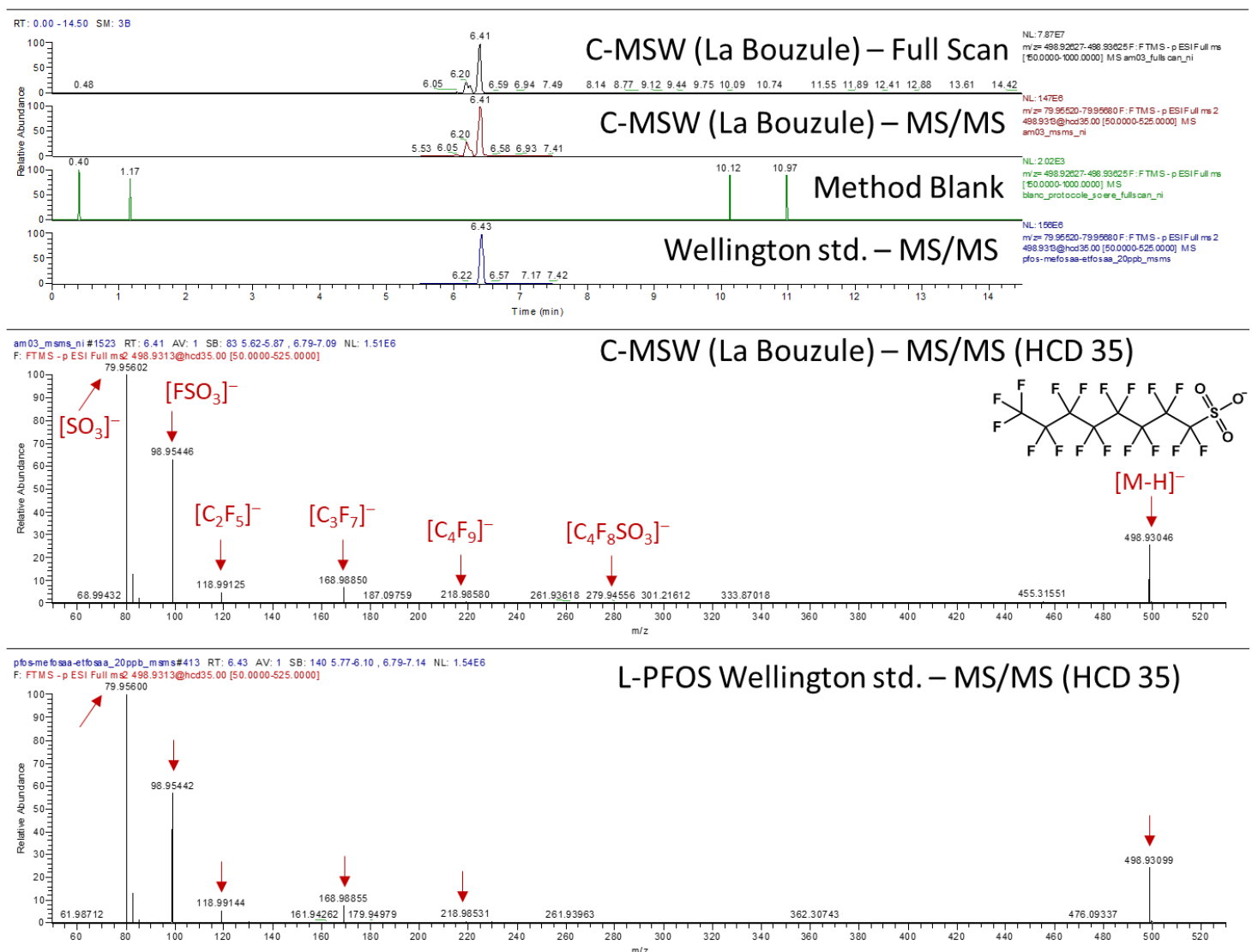

\begin{tabular}{|c|c|c|c|}
\hline & Theoretical $\mathrm{m} / \mathrm{z}$ & Observed $\mathrm{m} / \mathrm{z}$ & $\delta(\mathrm{ppm})$ \\
\hline Pseudomolecular ion $(\mathrm{M}-\mathrm{H})^{-}$ & 498.92967 & 498.93046 & 1.6 \\
\hline Fragment 1 & 79.95682 & 79.95602 & -10.0 \\
\hline Fragment 2 & 98.95522 & 98.95446 & -7.7 \\
\hline Fragment 3 & 168.98882 & 168.98850 & -1.9 \\
\hline Fragment 4 & 118.99201 & 118.99125 & -6.4 \\
\hline Fragment 5 & 218.98563 & 218.98580 & 0.8 \\
\hline Fragment 6 & 279.94404 & 279.94556 & 5.4 \\
\hline
\end{tabular}


(S2-b). H-PFOS, compost of green wastes and sewage sludge (C-GWS) from La Bouzule site. An aqueous film-forming foam (Light Water AFFF) was also analyzed for comparison.

\section{H-PFOS}

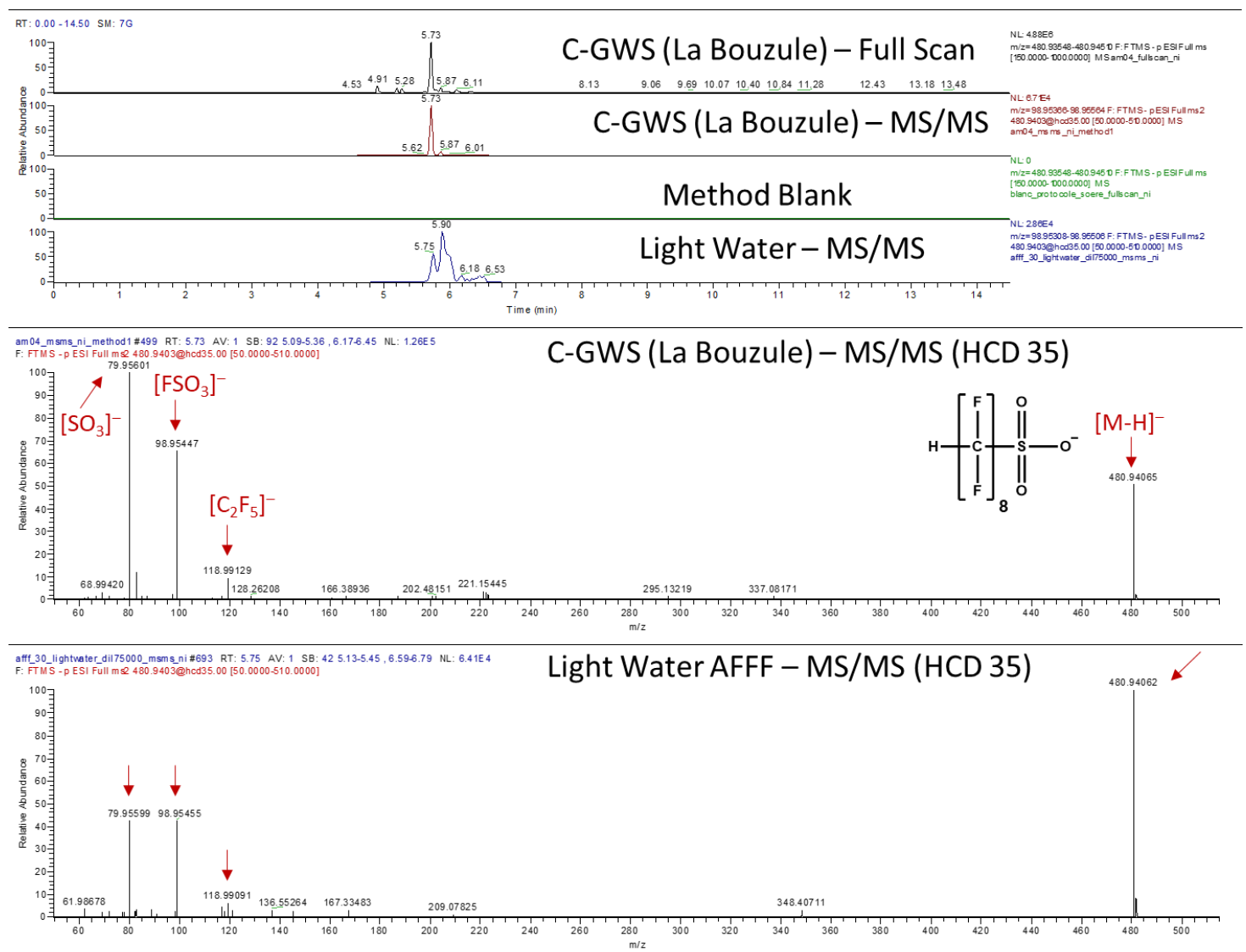

\begin{tabular}{|c|c|c|c|}
\hline & Theoretical $\mathrm{m} / \mathrm{z}$ & Observed $\mathrm{m} / \mathrm{z}$ & $\delta(\mathrm{ppm})$ \\
\hline Pseudomolecular ion $(\mathrm{M}-\mathrm{H})^{-}$ & 480.94037 & 480.94065 & 0.6 \\
\hline Fragment 1 & 79.95682 & 79.95601 & -10.1 \\
\hline Fragment 2 & 98.95522 & 98.95447 & -7.6 \\
\hline Fragment 3 & 118.99201 & 118.99129 & -6.1 \\
\hline
\end{tabular}


(S2-c). PFOSi, compost of residual municipal solid waste (C-MSW) from La Bouzule site.

\section{PFOSi}

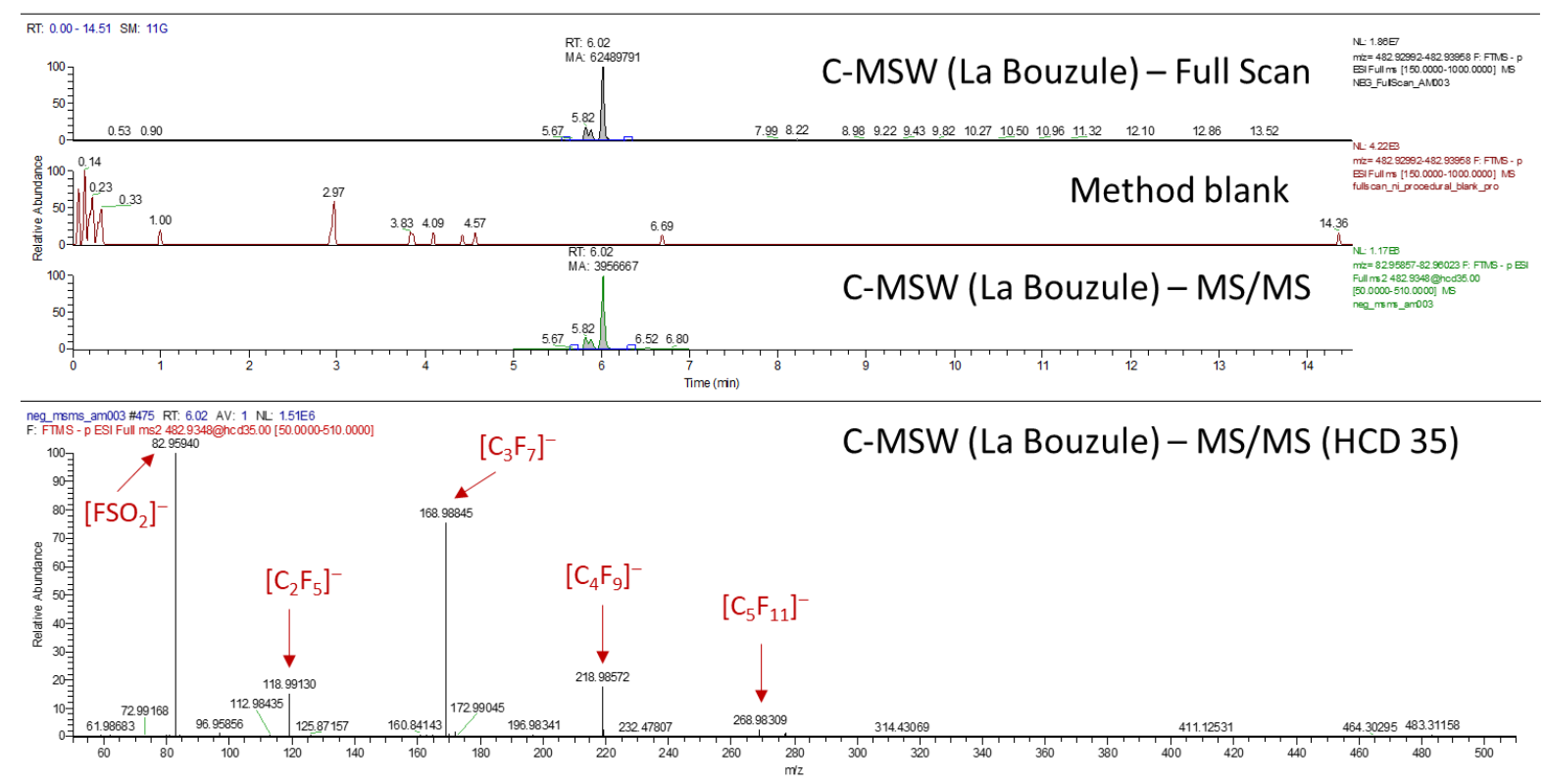

\begin{tabular}{|l|c|c|c|}
\hline & Theoretical $\mathrm{m} / \mathrm{z}$ & Observed $\mathrm{m} / \mathrm{z}$ & $\delta(\mathrm{ppm})$ \\
\hline Fragment 1 & 82.96030 & 82.95940 & -10.8 \\
\hline Fragment 2 & 168.98882 & 168.98845 & -2.2 \\
\hline Fragment 3 & 218.98563 & 218.98572 & 0.4 \\
\hline Fragment 4 & 118.99201 & 118.99130 & -6.0 \\
\hline Fragment 5 & 268.98243 & 268.98309 & 2.5 \\
\hline
\end{tabular}


(S2-d). 9n-F5S-PFSA, compost of green wastes and sewage sludge (C-GWS) from La Bouzule site. An aqueous film-forming foam (Light Water AFFF) was also analyzed for comparison.

\section{9n-F5S-PFSA}

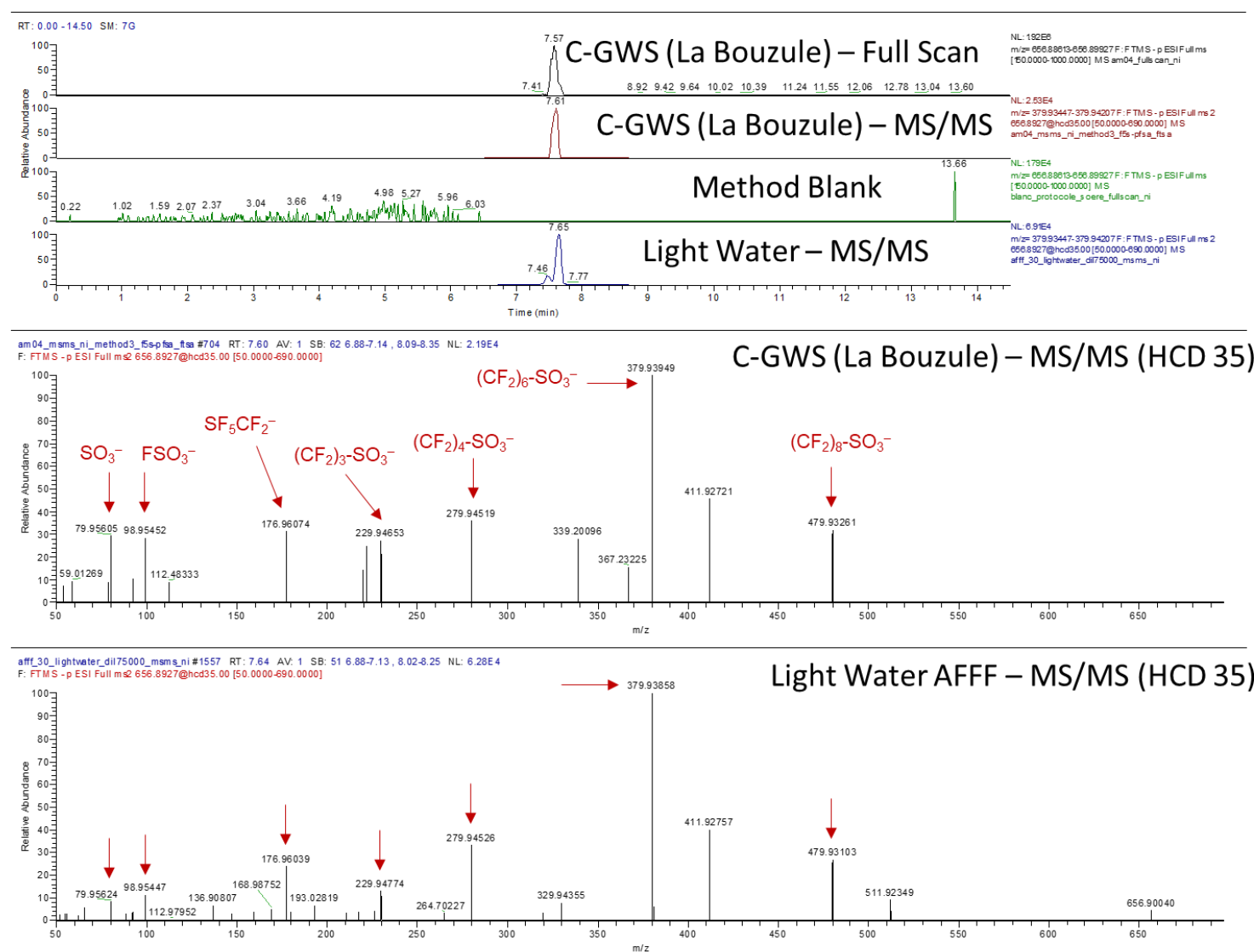

\begin{tabular}{|c|c|c|c|}
\hline & Theoretical $\mathrm{m} / \mathrm{z}$ & Observed $\mathrm{m} / \mathrm{z}$ & $\delta(\mathrm{ppm})$ \\
\hline Fragment 1 & 379.93765 & 379.93949 & 4.8 \\
\hline Fragment 2 & 279.94404 & 279.94519 & 4.1 \\
\hline Fragment 3 & 479.93126 & 479.93261 & 2.8 \\
\hline Fragment 4 & 176.96089 & 176.96074 & -0.8 \\
\hline Fragment 5 & 79.95682 & 79.95605 & -9.6 \\
\hline Fragment 6 & 98.95522 & 98.95452 & -7.1 \\
\hline Fragment 7 & 229.94723 & 229.94653 & -3.0 \\
\hline
\end{tabular}


(S2-e). FHxSAA, compost of green wastes and sewage sludge (C-GWS) from La Bouzule site. An aqueous film-forming foam (Light Water AFFF) was also analyzed for comparison.

\section{FHxSAA}

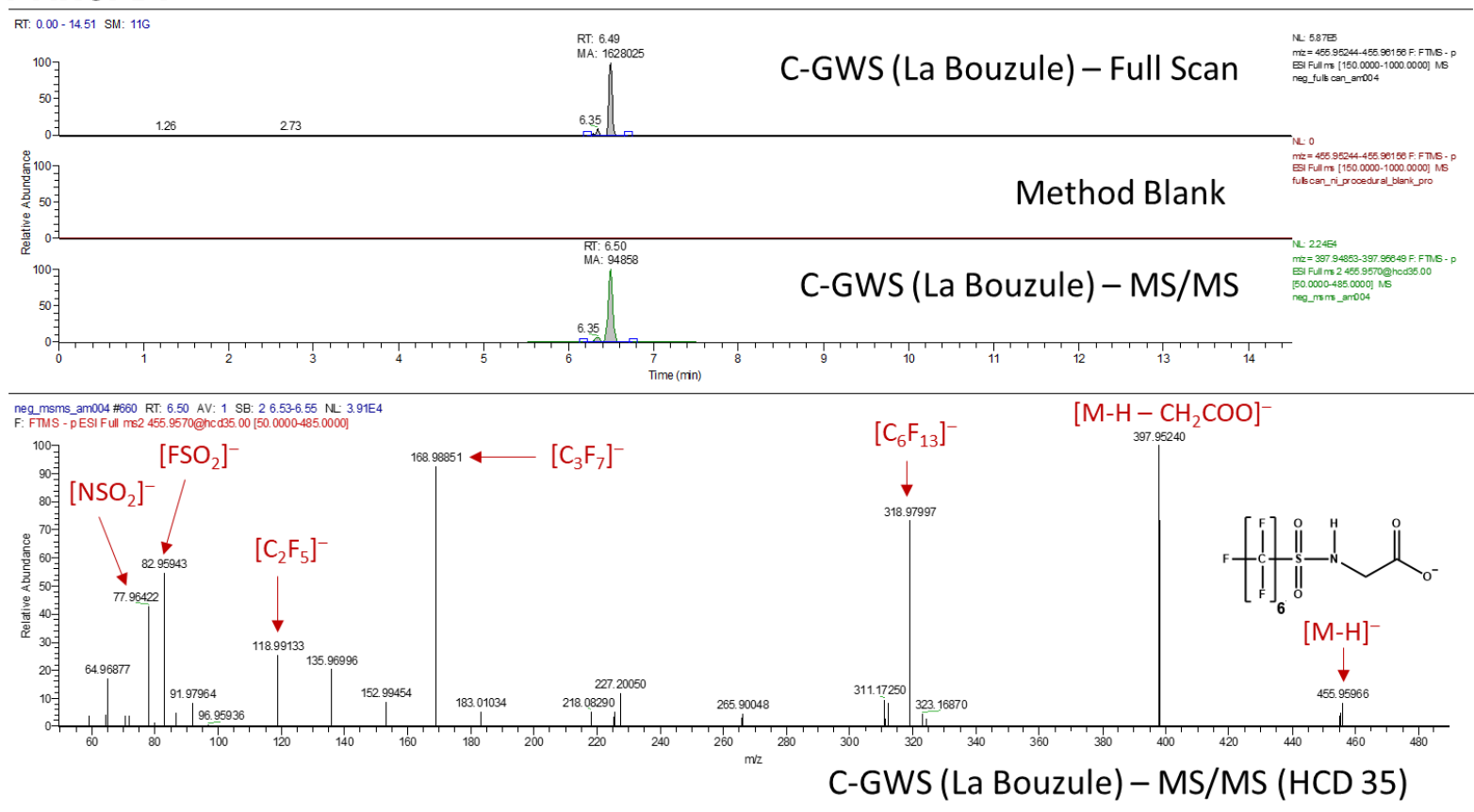

\begin{tabular}{|c|c|c|c|}
\hline & Theoretical $\mathrm{m} / \mathrm{z}$ & Observed $\mathrm{m} / \mathrm{z}$ & $\delta(\mathrm{ppm})$ \\
\hline Pseudomolecular ion $(\mathrm{M}-\mathrm{H})^{-}$ & 455.95752 & 455.95966 & 4.7 \\
\hline Fragment 1 & 397.95204 & 397.95240 & 0.9 \\
\hline Fragment 2 & 168.98882 & 168.98851 & -1.8 \\
\hline Fragment 3 & 318.97924 & 318.97997 & 2.3 \\
\hline Fragment 4 & 82.96030 & 82.95943 & -10.5 \\
\hline Fragment 5 & 77.96497 & 77.96422 & -9.6 \\
\hline Fragment 6 & 118.99201 & 118.99133 & -5.7 \\
\hline
\end{tabular}


(S2-f). MeFNSAA, sewage sludge (SLU) from La Bouzule site.

\section{MeFNSAA}
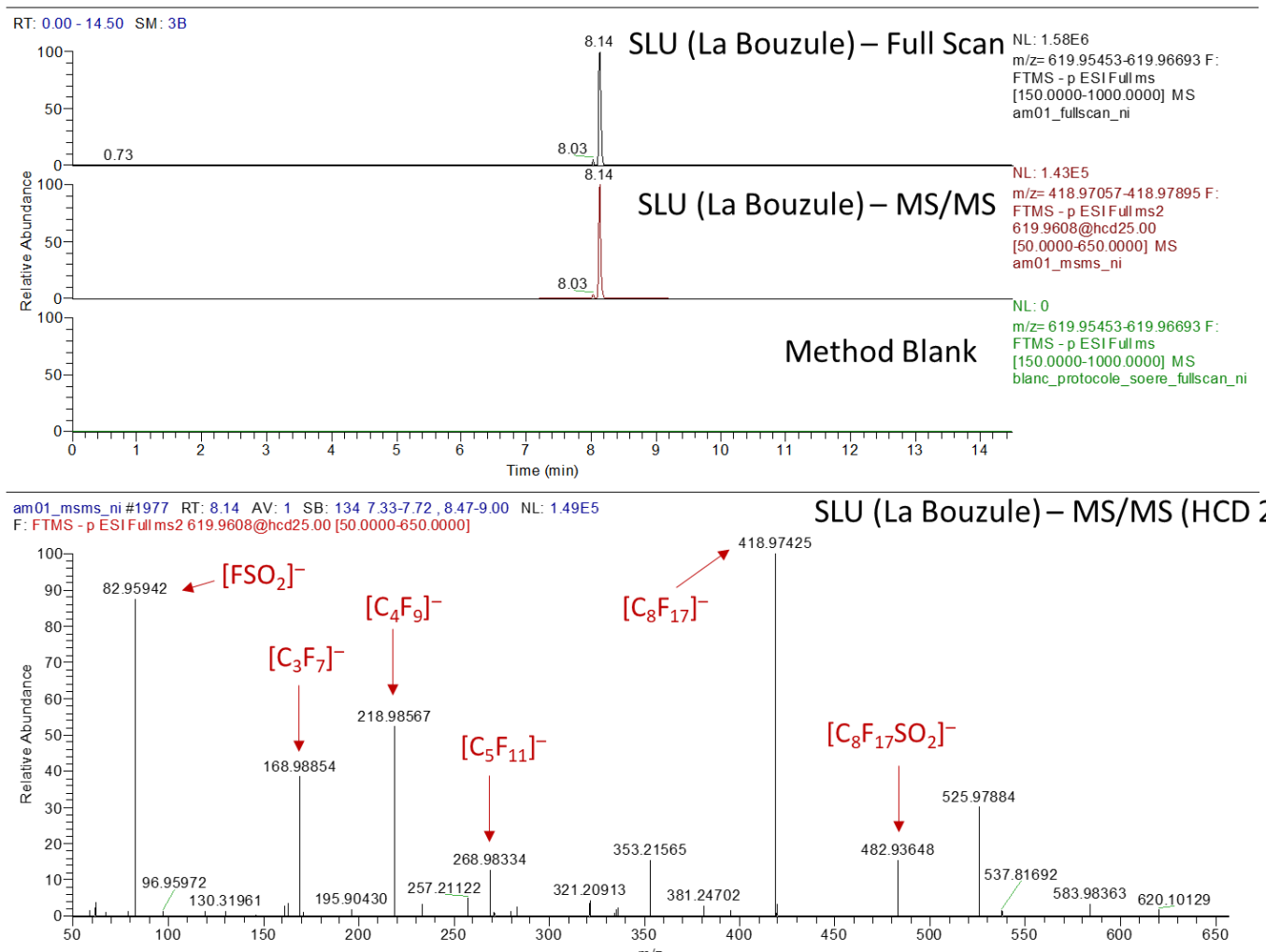

\begin{tabular}{|l|c|c|c|}
\hline & Theoretical $\mathrm{m} / \mathrm{z}$ & Observed $\mathrm{m} / \mathrm{z}$ & $\delta(\mathrm{ppm})$ \\
\hline Fragment 1 & 418.97285 & 418.97425 & 3.3 \\
\hline Fragment 2 & 82.96030 & 82.95942 & -10.6 \\
\hline Fragment 3 & 218.98563 & 218.98567 & 0.2 \\
\hline Fragment 4 & 168.98882 & 168.9884 & -2.5 \\
\hline Fragment 5 & 482.93475 & 482.93648 & 3.6 \\
\hline Fragment 6 & 268.98243 & 268.98334 & 3.4 \\
\hline
\end{tabular}


(S2-g). EtFHxSAA, compost of residual municipal solid waste (C-MSW) from La Bouzule site.

\section{EtFHxSAA}

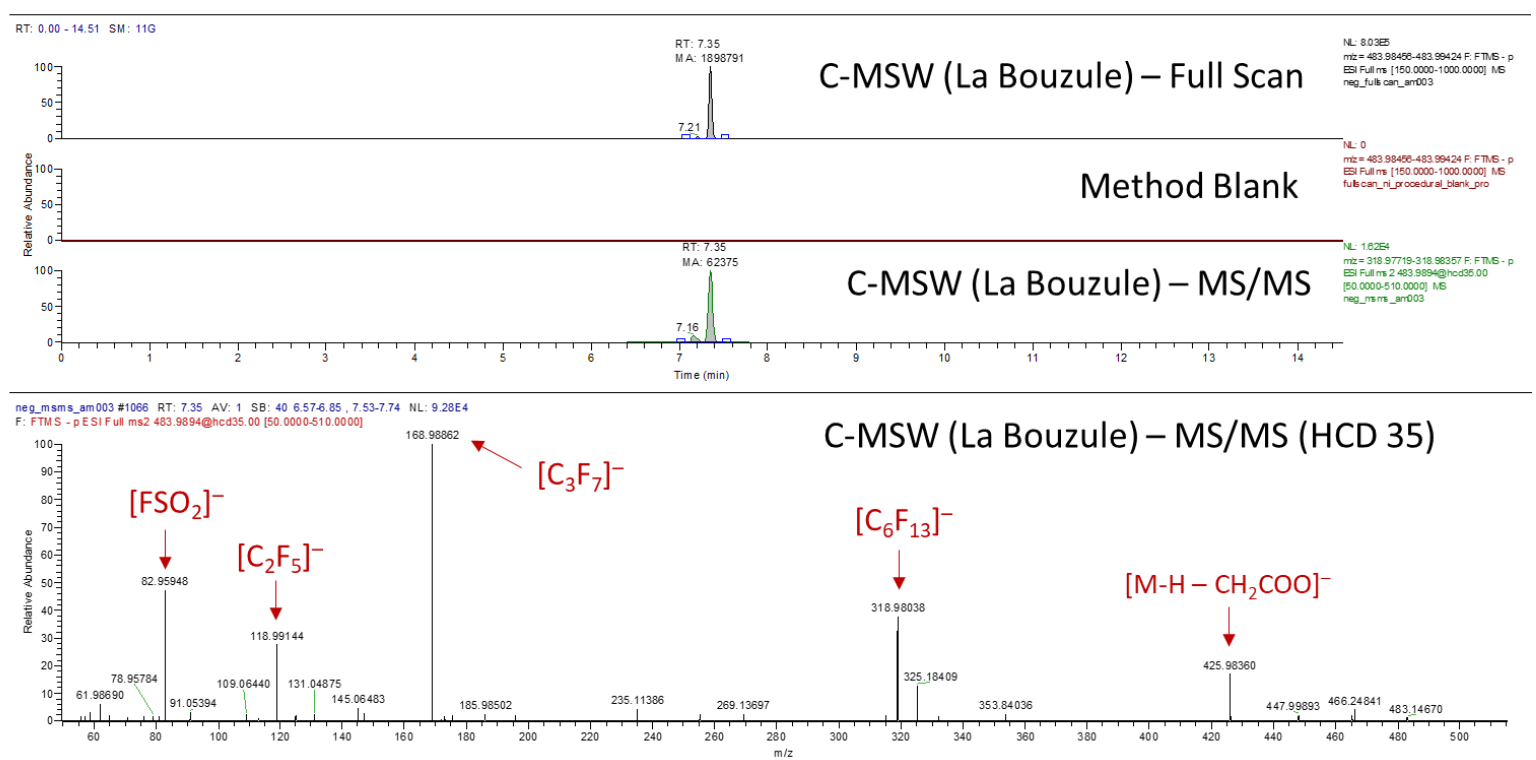

\begin{tabular}{|c|c|c|c|}
\hline & Theoretical $\mathrm{m} / \mathrm{z}$ & Observed $\mathrm{m} / \mathrm{z}$ & $\delta(\mathrm{ppm})$ \\
\hline Fragment 1 & 168.98882 & 168.98862 & -1.2 \\
\hline Fragment 2 & 82.96030 & 82.95948 & -9.9 \\
\hline Fragment 3 & 318.97924 & 318.98038 & 3.6 \\
\hline Fragment 4 & 118.99201 & 118.99144 & -4.8 \\
\hline Fragment 5 & 425.98334 & 425.98360 & 0.6 \\
\hline
\end{tabular}


(S2-h). EtFHpSAA, compost of residual municipal solid waste (C-MSW) from La Bouzule site.

\section{EtFHpSAA}

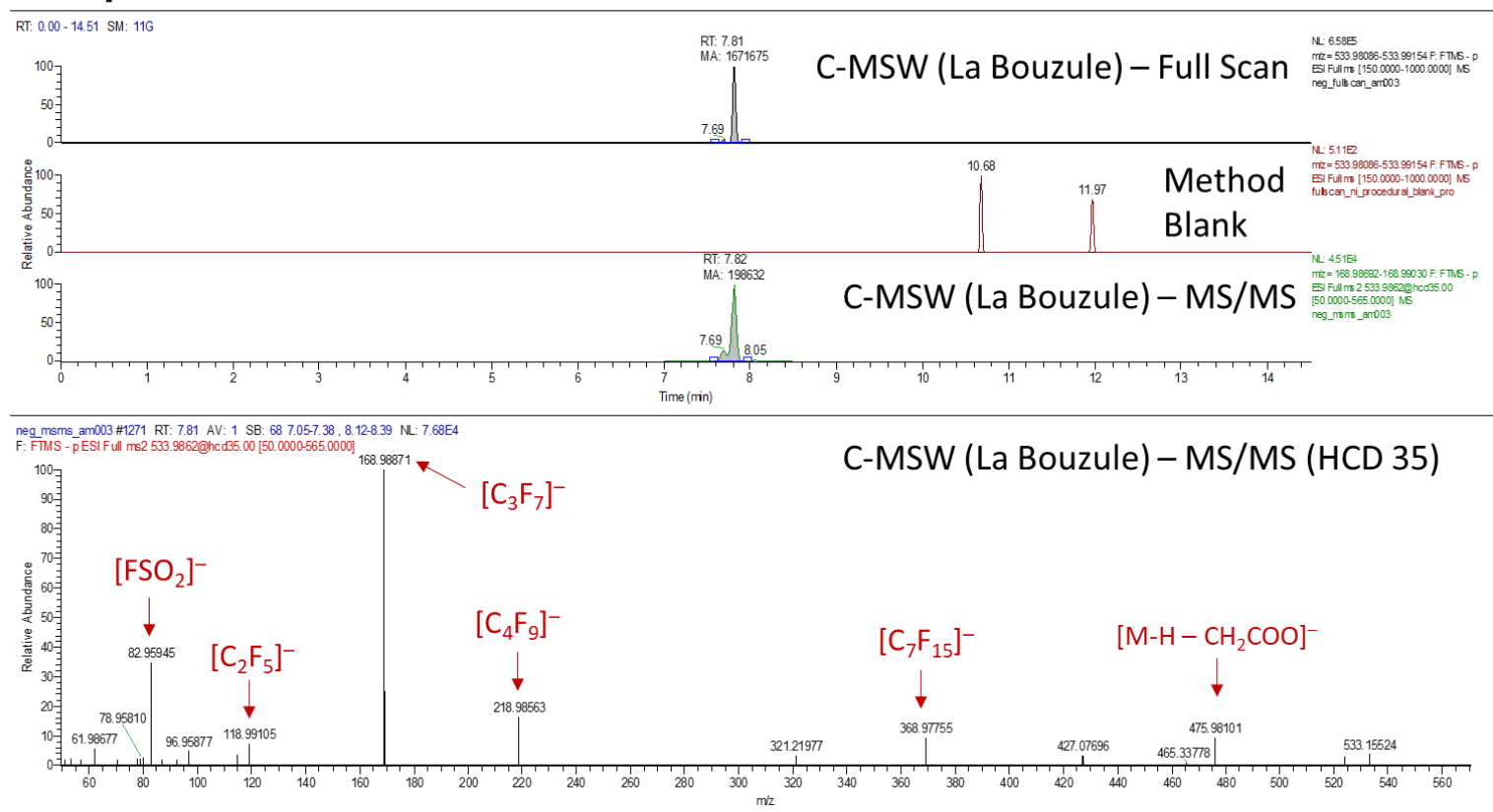

\begin{tabular}{|l|c|c|c|}
\hline & Theoretical $\mathrm{m} / \mathrm{z}$ & Observed $\mathrm{m} / \mathrm{z}$ & $\delta(\mathrm{ppm})$ \\
\hline Fragment 1 & 168.98882 & 168.98871 & -0.7 \\
\hline Fragment 2 & 82.96030 & 82.95945 & -10.2 \\
\hline Fragment 3 & 218.98563 & 218.98563 & 0.0 \\
\hline Fragment 4 & 368.97605 & 368.97755 & 4.1 \\
\hline Fragment 5 & 475.98015 & 475.98101 & 1.8 \\
\hline Fragment 6 & 118.99201 & 118.99105 & -8.1 \\
\hline
\end{tabular}


(S2-i). EtFOSAA, sewage sludge (SLU) from La Bouzule site. A certified standard (from Wellington Labs) was also analyzed for comparison.

\section{EtFOSAA}

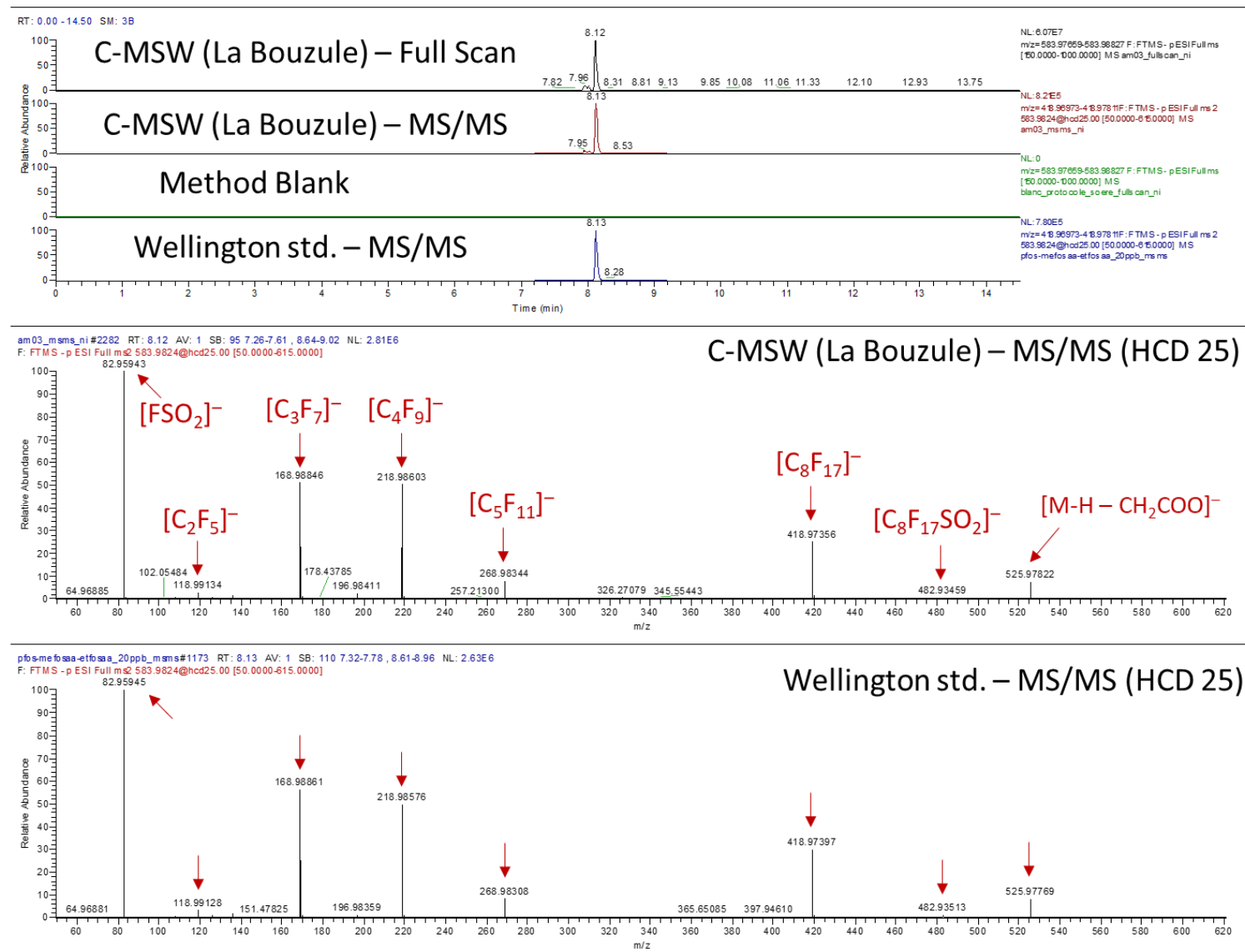

\begin{tabular}{|c|c|c|c|}
\hline & Theoretical $\mathrm{m} / \mathrm{z}$ & Observed $\mathrm{m} / \mathrm{z}$ & $\delta(\mathrm{ppm})$ \\
\hline Fragment 1 & 82.96030 & 82.95945 & -10.2 \\
\hline Fragment 2 & 168.98882 & 168.98861 & -1.2 \\
\hline Fragment 3 & 218.98563 & 218.98576 & 0.6 \\
\hline Fragment 4 & 418.97285 & 418.97397 & 2.7 \\
\hline Fragment 5 & 268.98243 & 268.98308 & 2.4 \\
\hline Fragment 6 & 525.97695 & 525.97769 & 1.4 \\
\hline Fragment 7 & 118.99201 & 118.99128 & -6.1 \\
\hline Fragment 8 & 482.93475 & 482.93513 & 0.8 \\
\hline
\end{tabular}


(S2-j). 6:2 FTSA-PrA (6:2 fluorotelomer sulfonamide), compost of green wastes and sewage sludge (C-GWS) from QualiAgro site.

\section{6:2 fluorotelomer sulfonamide}
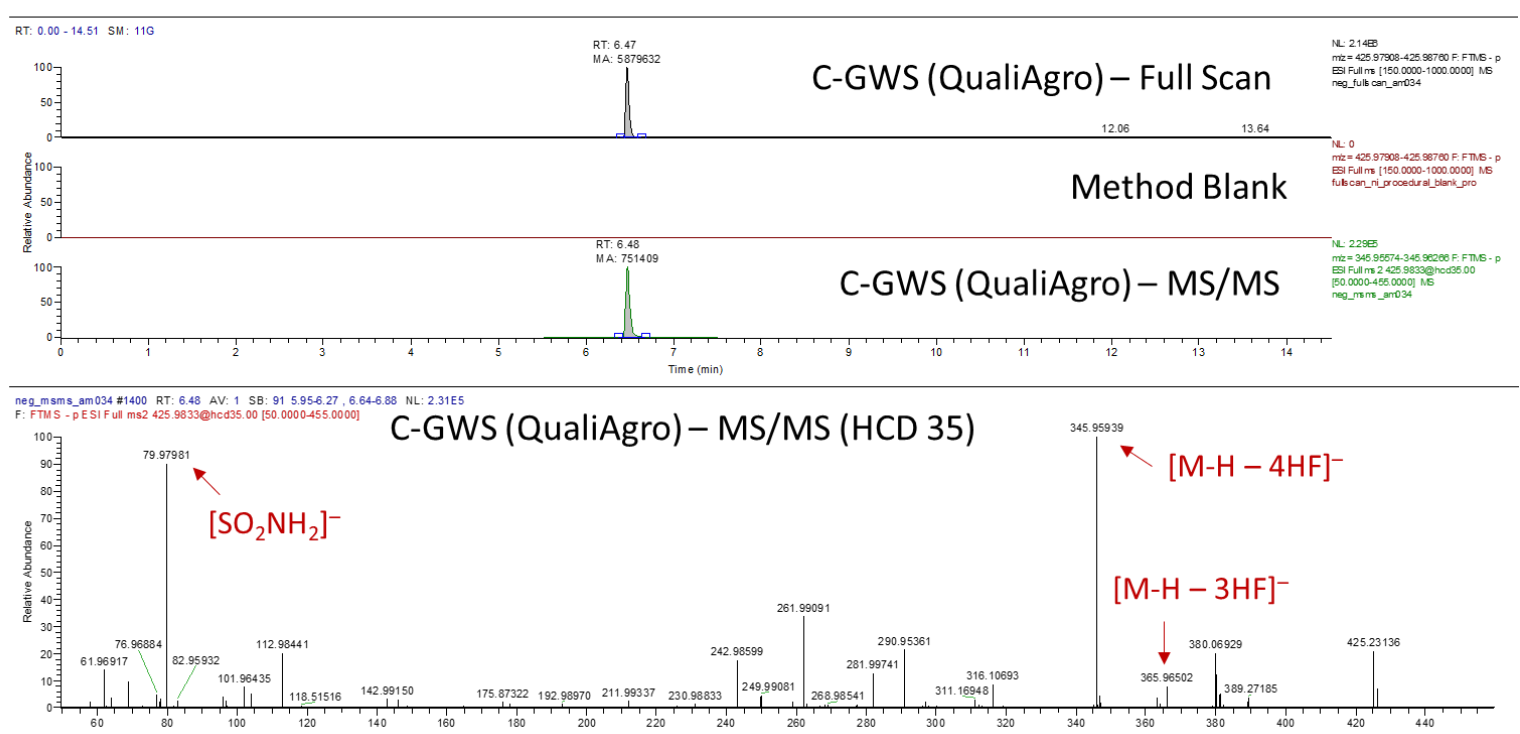

\begin{tabular}{|l|c|c|c|}
\hline & Theoretical $\mathrm{m} / \mathrm{z}$ & Observed $\mathrm{m} / \mathrm{z}$ & $\delta(\mathrm{ppm})$ \\
\hline Fragment 1 & 345.95843 & 345.95939 & 2.8 \\
\hline Fragment 2 & 79.98062 & 79.97981 & -10.1 \\
\hline Fragment 3 & 365.96465 & 365.96502 & 1.0 \\
\hline
\end{tabular}


(S2-k). 9:3 acid (9:3 FTCA), compost of green wastes and sewage sludge (C-GWS) from PROspective site.

\section{9:3 acid (9:3 FTCA)}

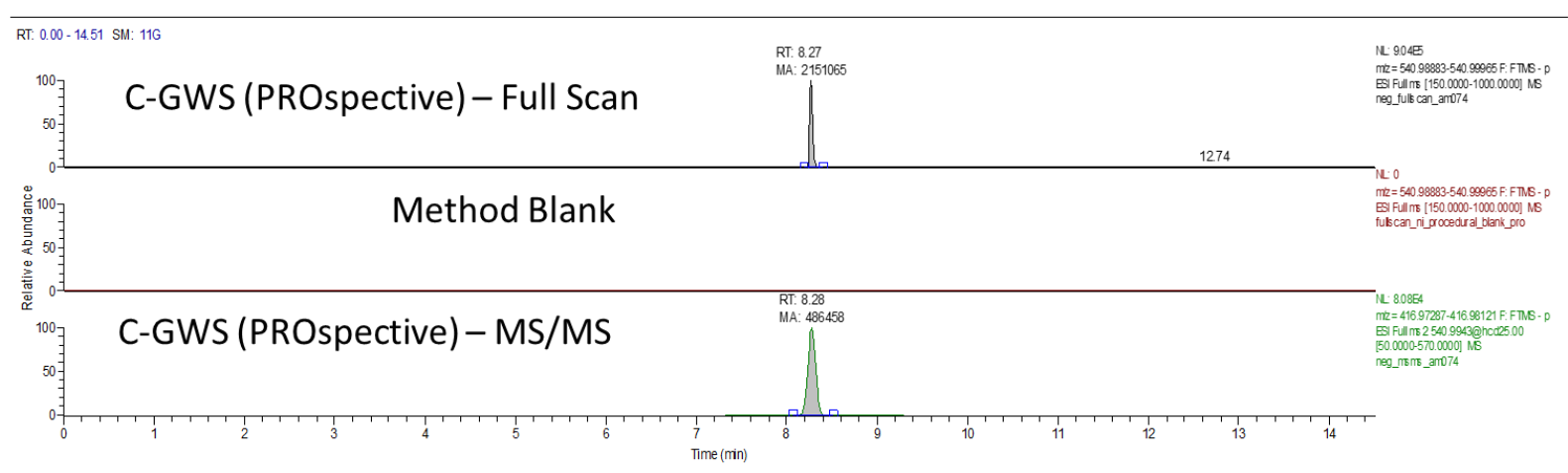

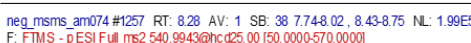

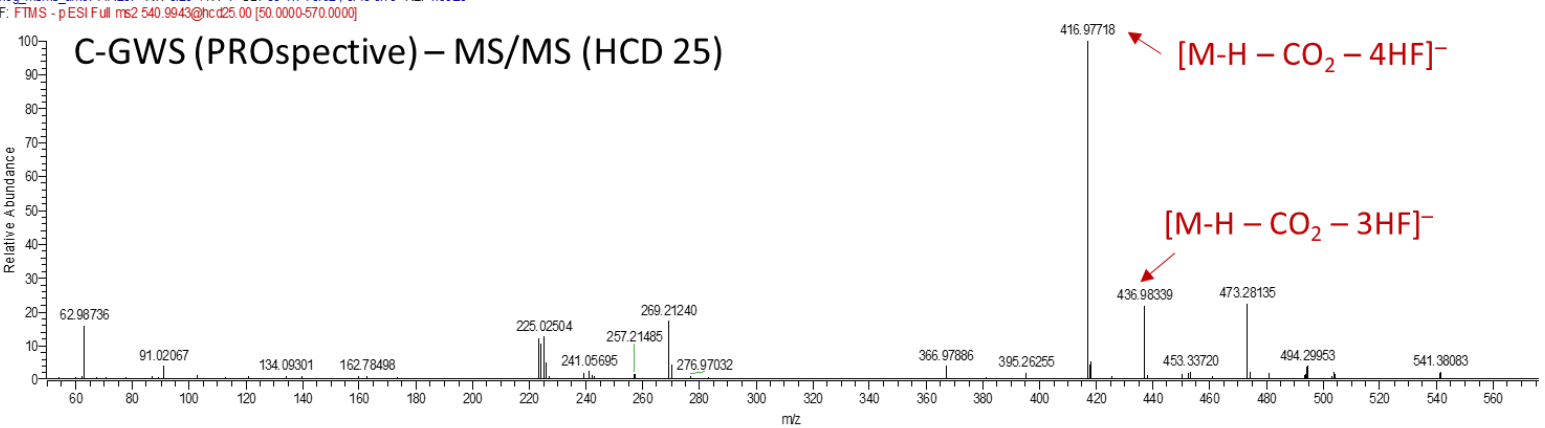

\begin{tabular}{|c|c|c|c|}
\hline & Theoretical $\mathrm{m} / \mathrm{z}$ & Observed $\mathrm{m} / \mathrm{z}$ & $\delta(\mathrm{ppm})$ \\
\hline Fragment 1 & 416.97604 & 416.97718 & 2.7 \\
\hline Fragment 2 & 436.98227 & 436.98339 & 2.6 \\
\hline
\end{tabular}


(S2-I). 11:3 acid (11:3 FTCA), compost of green wastes and sewage sludge (C-GWS) from PROspective site.

\section{1:3 acid (11:3 FTCA)}

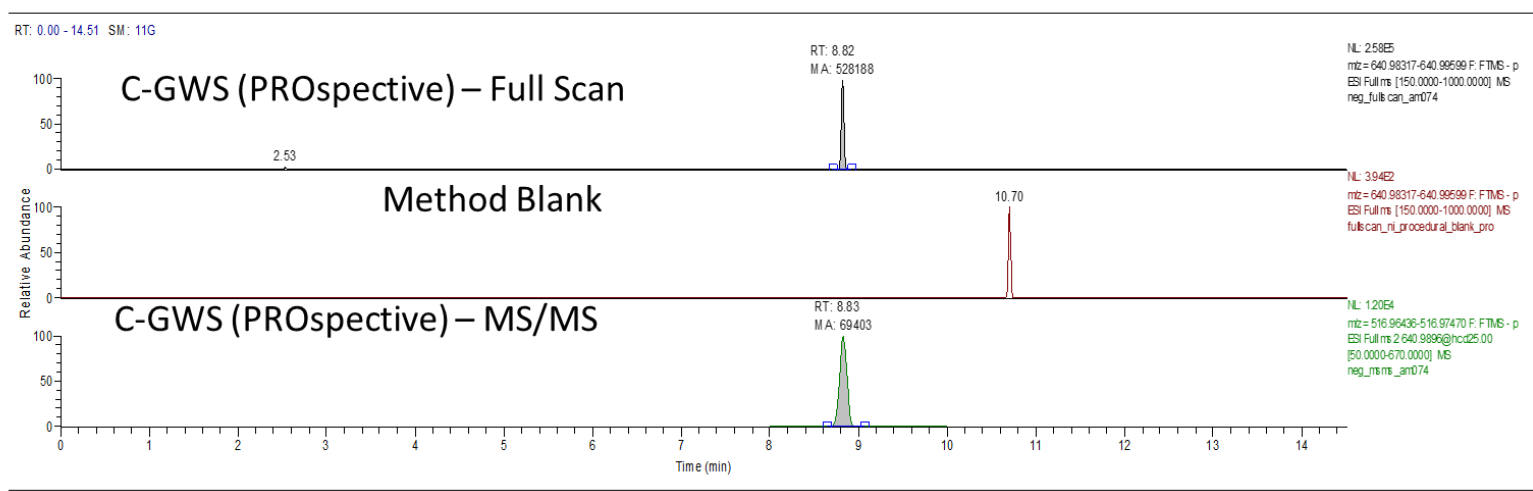

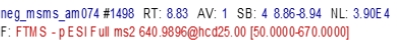

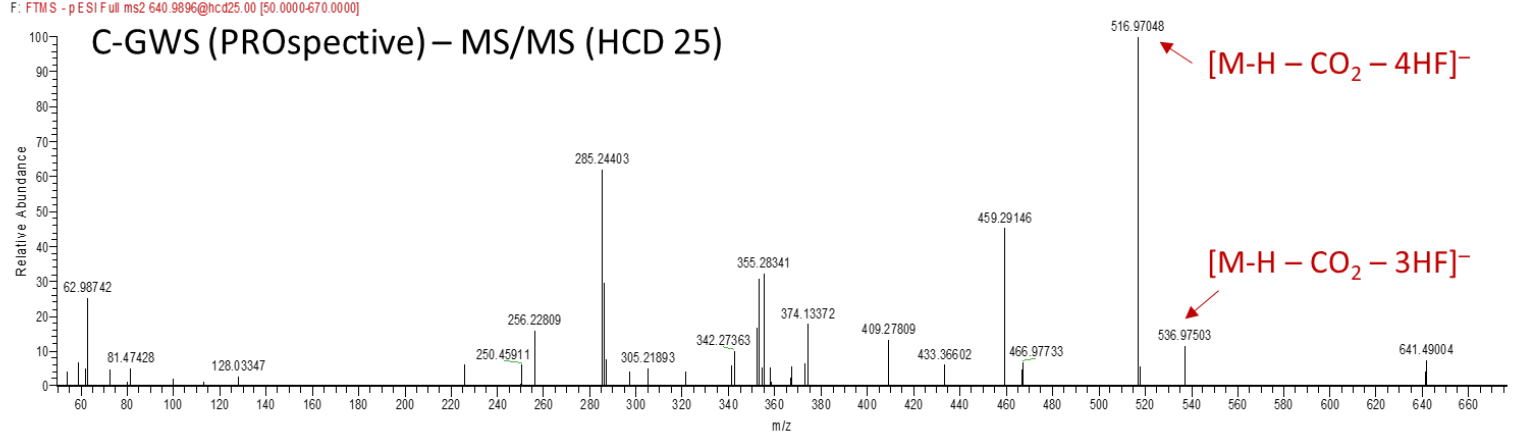

\begin{tabular}{|c|c|c|c|}
\hline & Theoretical $\mathrm{m} / \mathrm{z}$ & Observed $\mathrm{m} / \mathrm{z}$ & $\delta(\mathrm{ppm})$ \\
\hline Fragment 1 & 516.96965 & 516.97048 & 1.6 \\
\hline Fragment 2 & 536.97588 & 536.97503 & -1.6 \\
\hline
\end{tabular}


(S2-m). 6:2 FTSO-PrAdDiMePrS (6:2 FTSAS-sulfoxide), compost of residual municipal solid waste (C-MSW) from QualiAgro site.

\section{6:2 FTSO-PrAdDiMePrS (6:2 FTSAS-sulfoxide)}

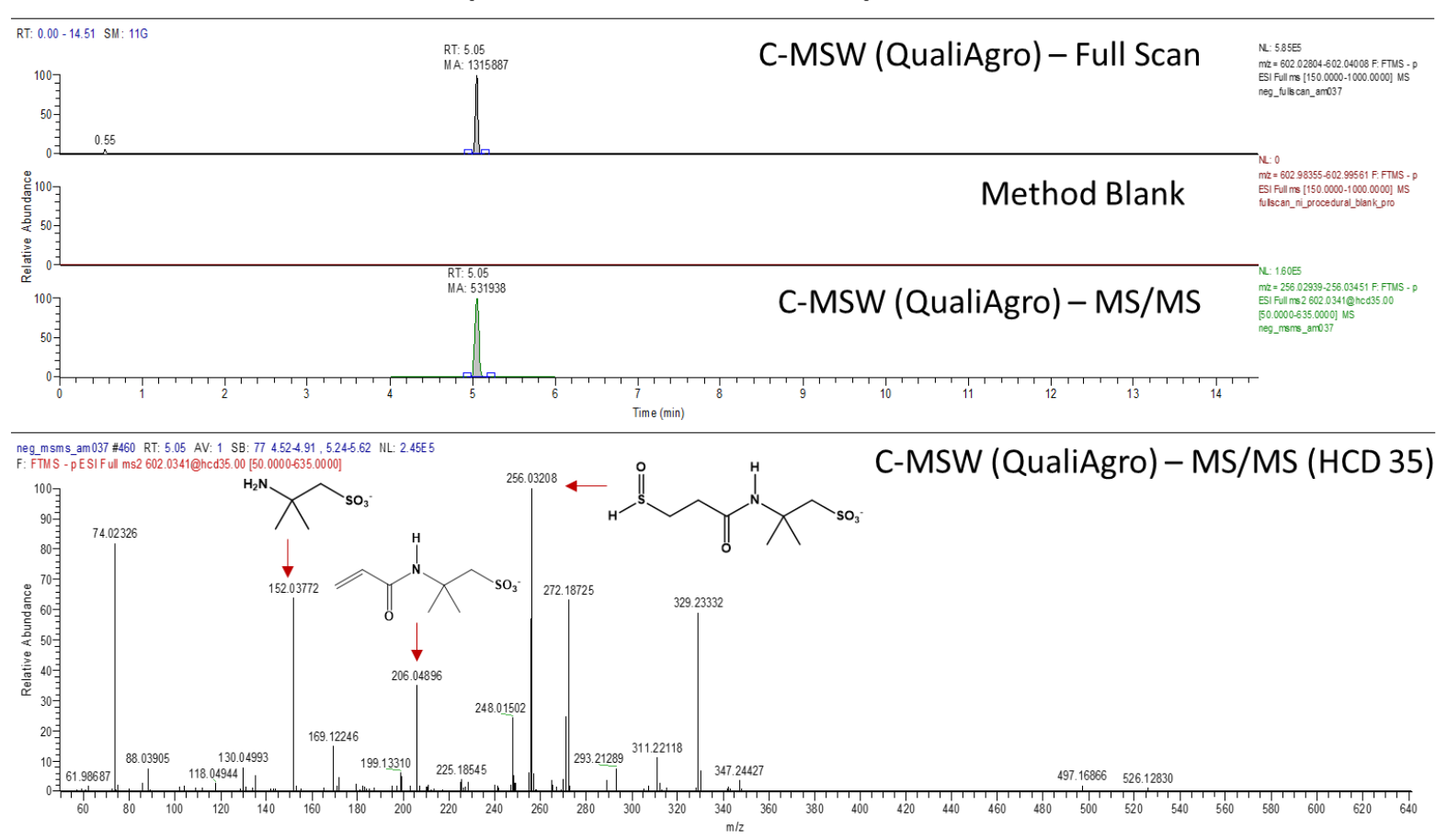

\begin{tabular}{|c|c|c|c|}
\hline & Theoretical $\mathrm{m} / \mathrm{z}$ & Observed $\mathrm{m} / \mathrm{z}$ & $\delta(\mathrm{ppm})$ \\
\hline Fragment 1 & 256.03134 & 256.03208 & 2.9 \\
\hline Fragment 2 & 152.03814 & 152.03772 & -2.8 \\
\hline Fragment 3 & 206.04870 & 206.04896 & 1.3 \\
\hline
\end{tabular}


(S2-n). 6:2 FTSO2-PrAdDiMeEtS (6:2 FTSAS-sulfone), compost of residual municipal solid waste (C-MSW) from QualiAgro site.

\section{6:2 FTSO2-PrAdDiMeEtS (6:2 FTSAS-sulfone)}

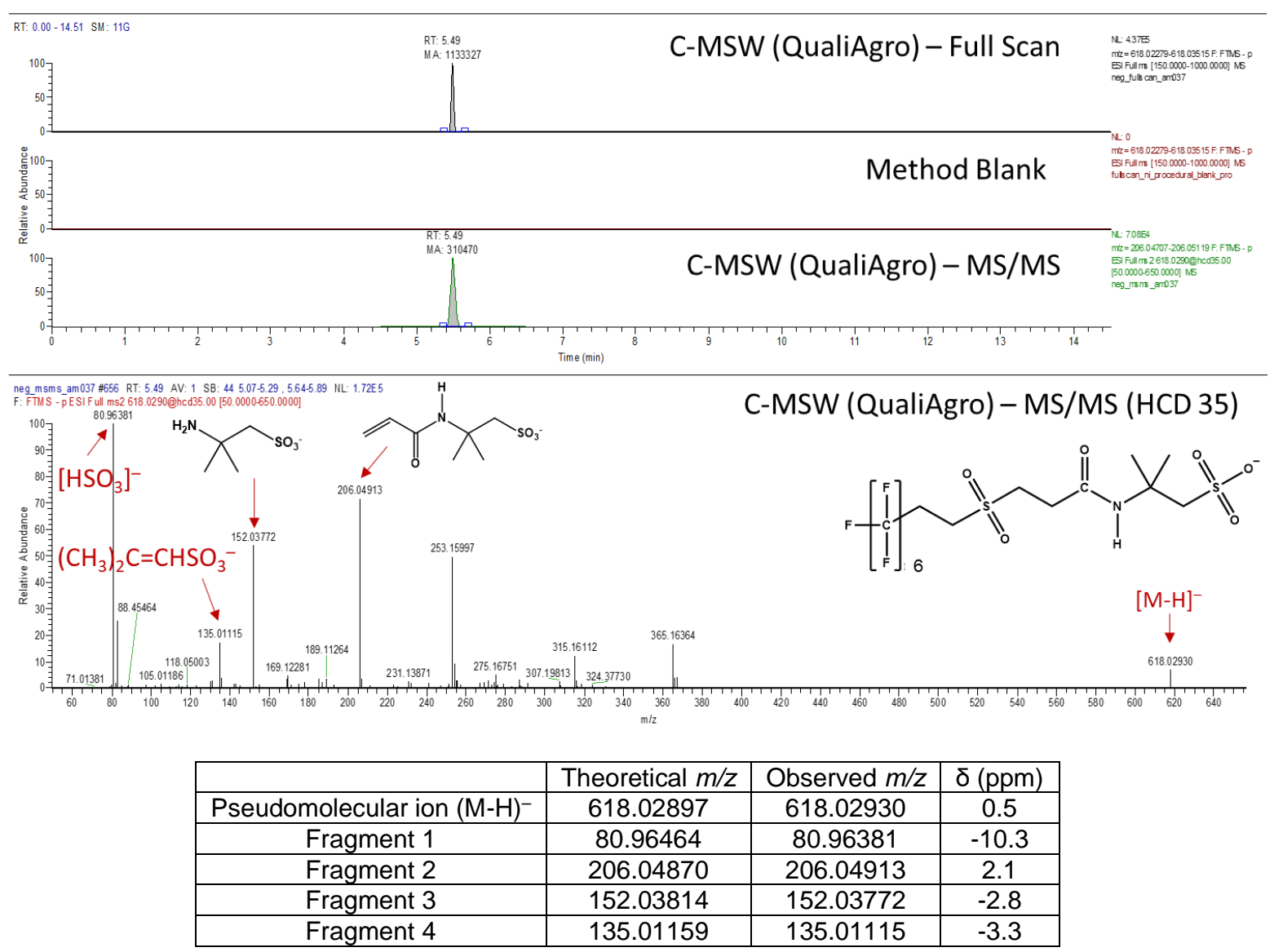


(S2-0). 6:6 PhiA, compost of green wastes and sewage sludge (C-GWS) from La Bouzule site.

\section{6:6 PhiA}
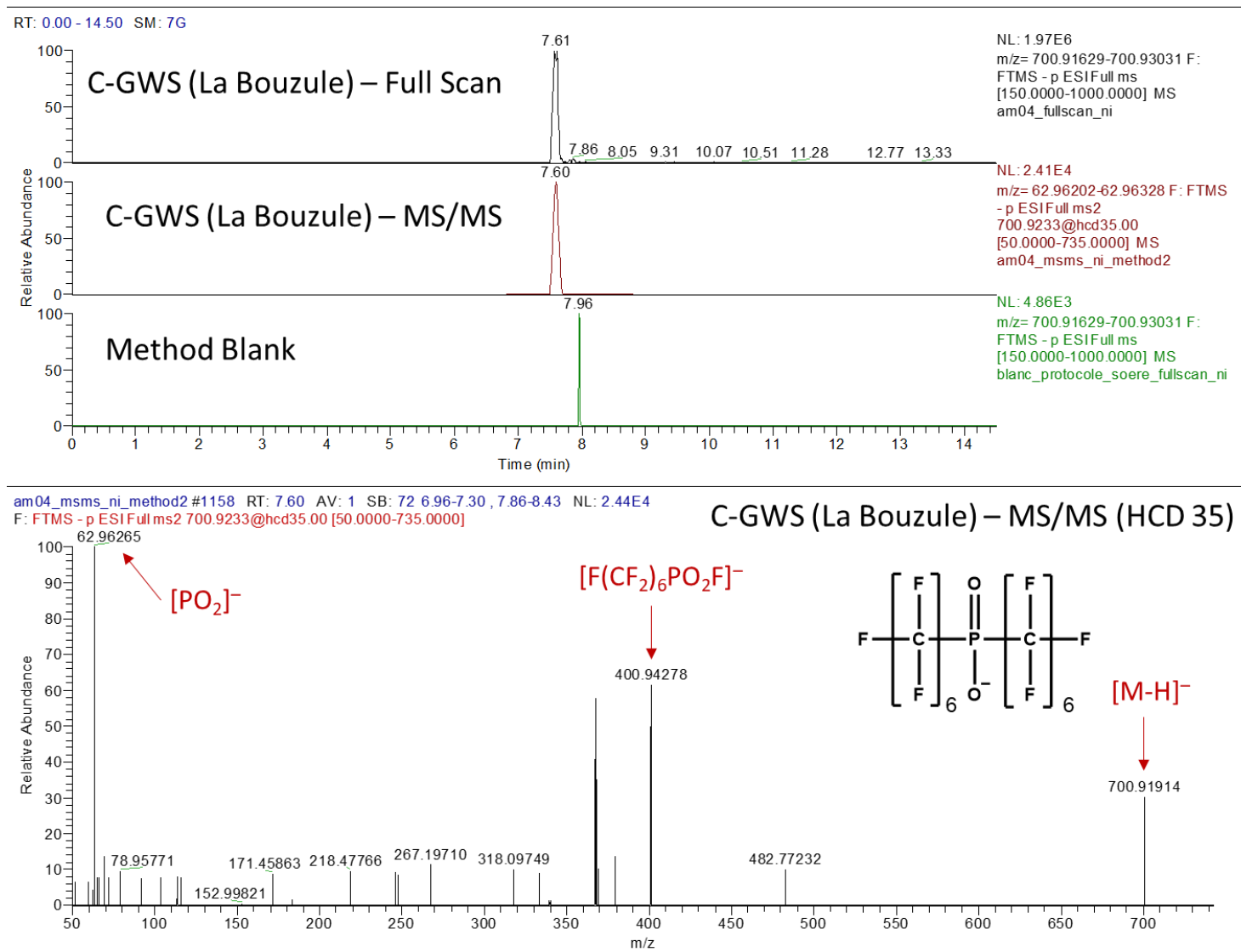

\begin{tabular}{|c|c|c|c|}
\hline & Theoretical $m / z$ & Observed $m / z$ & $\delta(\mathrm{ppm})$ \\
\hline Pseudomolecular ion $(\mathrm{M}-\mathrm{H})^{-}$ & 700.92207 & 700.91914 & -4.2 \\
\hline Fragment 1 & 62.96359 & 62.96265 & -14.9 \\
\hline Fragment 2 & 400.94123 & 400.94278 & 3.9 \\
\hline
\end{tabular}


(S2-p). T-AmPr-FOSA (PFOSAmS), compost of green wastes and sewage sludge (C-GWS) from La Bouzule site. A custom-order reference standard (from Fluobon) was also analyzed for comparison.

\section{T-AmPr-FOSA (PFOSAmS)}
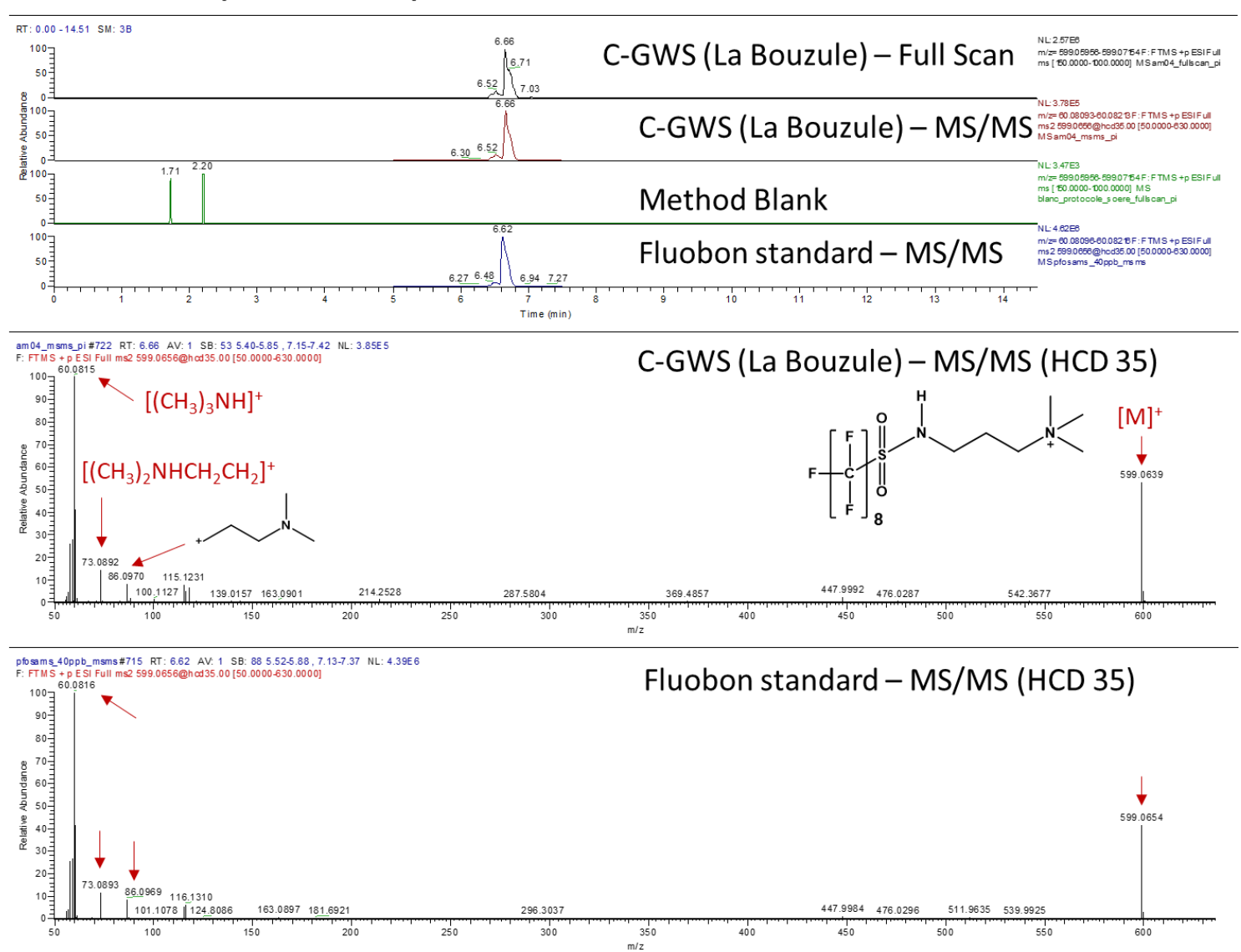

\begin{tabular}{|c|c|c|c|}
\hline & Theoretical $\mathrm{m} / \mathrm{z}$ & Observed $\mathrm{m} / \mathrm{z}$ & $\delta(\mathrm{ppm})$ \\
\hline Pseudomolecular ion $(\mathrm{M})^{+}$ & 599.0661 & 599.0639 & -3.7 \\
\hline Fragment 1 & 60.0813 & 60.0815 & 3.3 \\
\hline Fragment 2 & 73.0891 & 73.0892 & 1.4 \\
\hline Fragment 3 & 86.0969 & 86.0970 & 1.2 \\
\hline
\end{tabular}


(S2-q). AmPr-FOSA (PFOSAm), compost of green wastes and sewage sludge (C-GWS) from La Bouzule site. A custom-order reference standard (from Fluobon) was also analyzed for comparison.

\section{AmPr-FOSA (PFOSAm)}
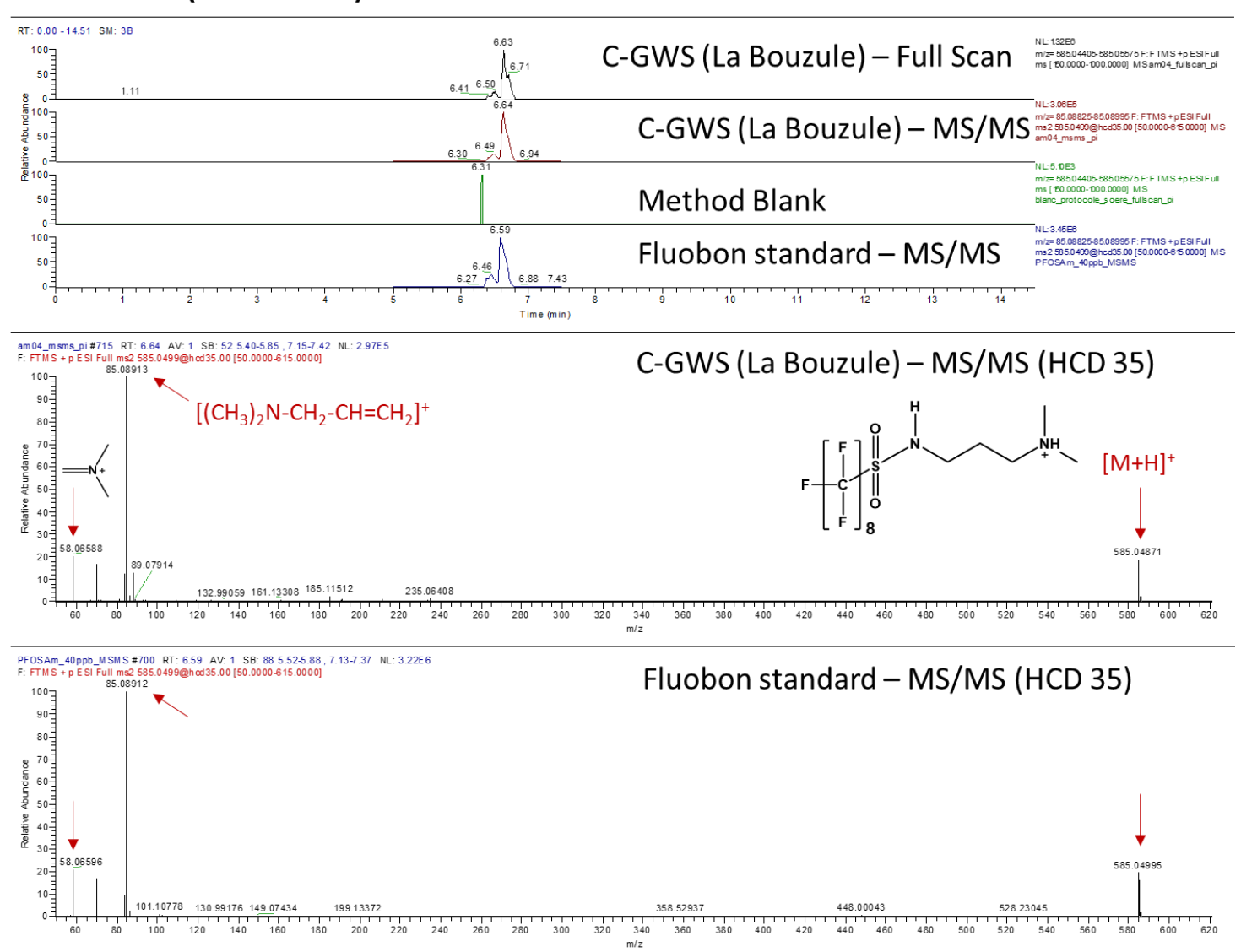

\begin{tabular}{|c|c|c|c|}
\hline & Theoretical $m / z$ & Observed $m / z$ & $\delta(p p m)$ \\
\hline Pseudomolecular ion $(\mathrm{M}+\mathrm{H})^{+}$ & 585.05045 & 585.04871 & -3.0 \\
\hline Fragment 1 & 85.08915 & 85.08912 & -0.4 \\
\hline Fragment 2 & 58.06567 & 58.06596 & 5.0 \\
\hline
\end{tabular}


(S2-r). CMeAmPr-FOSA (PFOSB), compost of green wastes and sewage sludge (C-GWS) from La Bouzule site. A custom-order reference standard (from Fluobon) was also analyzed for comparison.

\section{CMeAmPr-FOSA (PFOSB)}
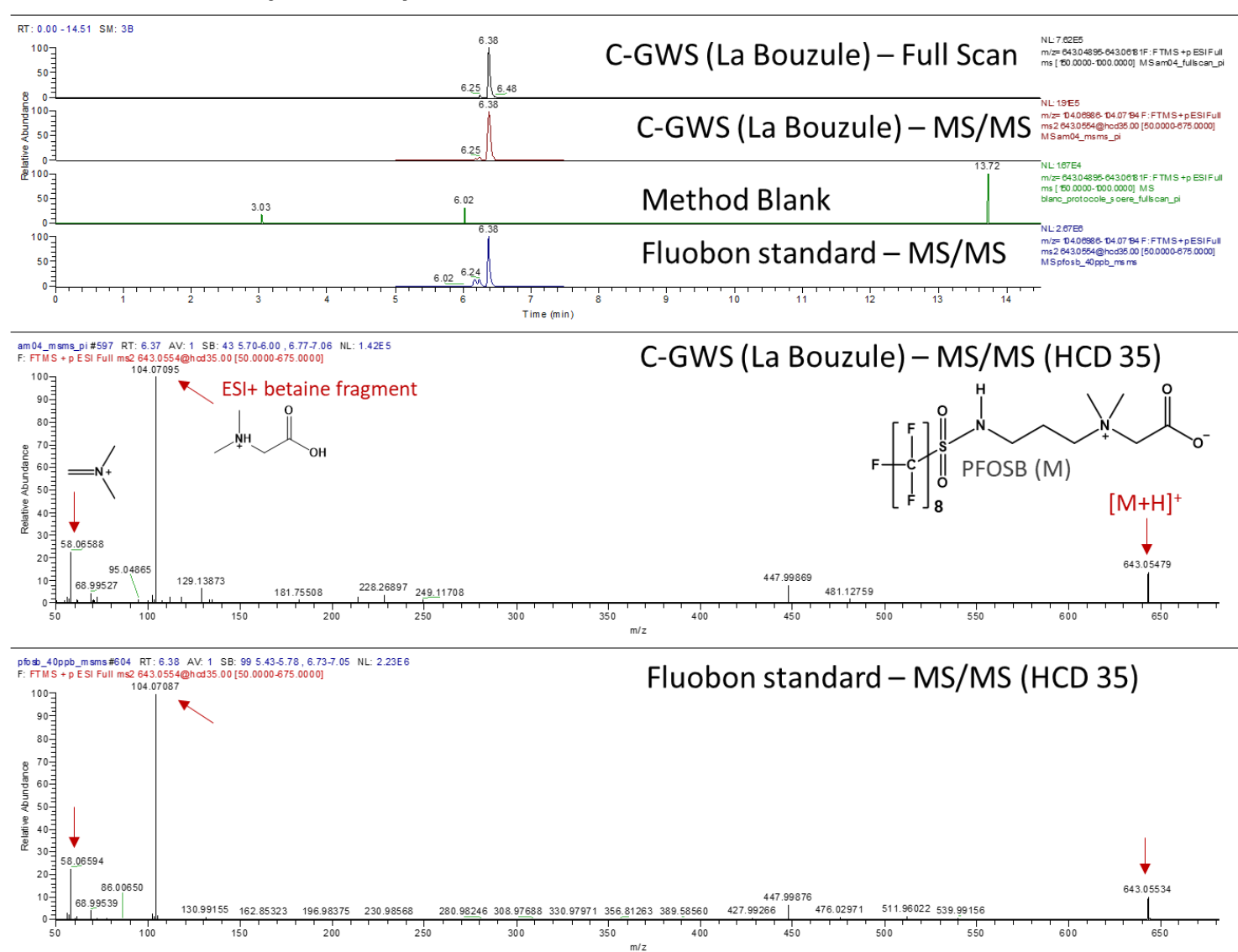

\begin{tabular}{|c|c|c|c|}
\hline & Theoretical $\mathrm{m} / \mathrm{z}$ & Observed $\mathrm{m} / \mathrm{z}$ & $\delta(\mathrm{ppm})$ \\
\hline Pseudomolecular ion $(\mathrm{M}+\mathrm{H})^{+}$ & 643.05593 & 643.05479 & -1.8 \\
\hline Fragment 1 & 104.07115 & 104.07095 & -1.9 \\
\hline Fragment 2 & 58.06567 & 58.06588 & 3.6 \\
\hline
\end{tabular}


(S2-s). 6:2 FTSA-PrB (6:2 FTAB), compost of residual municipal solid wastes (C-MSW) from QualiAgro site. A certified standard (from Wellington Labs) was also analyzed for comparison.

\section{6:2 FTSA-PrB (6:2 FTAB)}

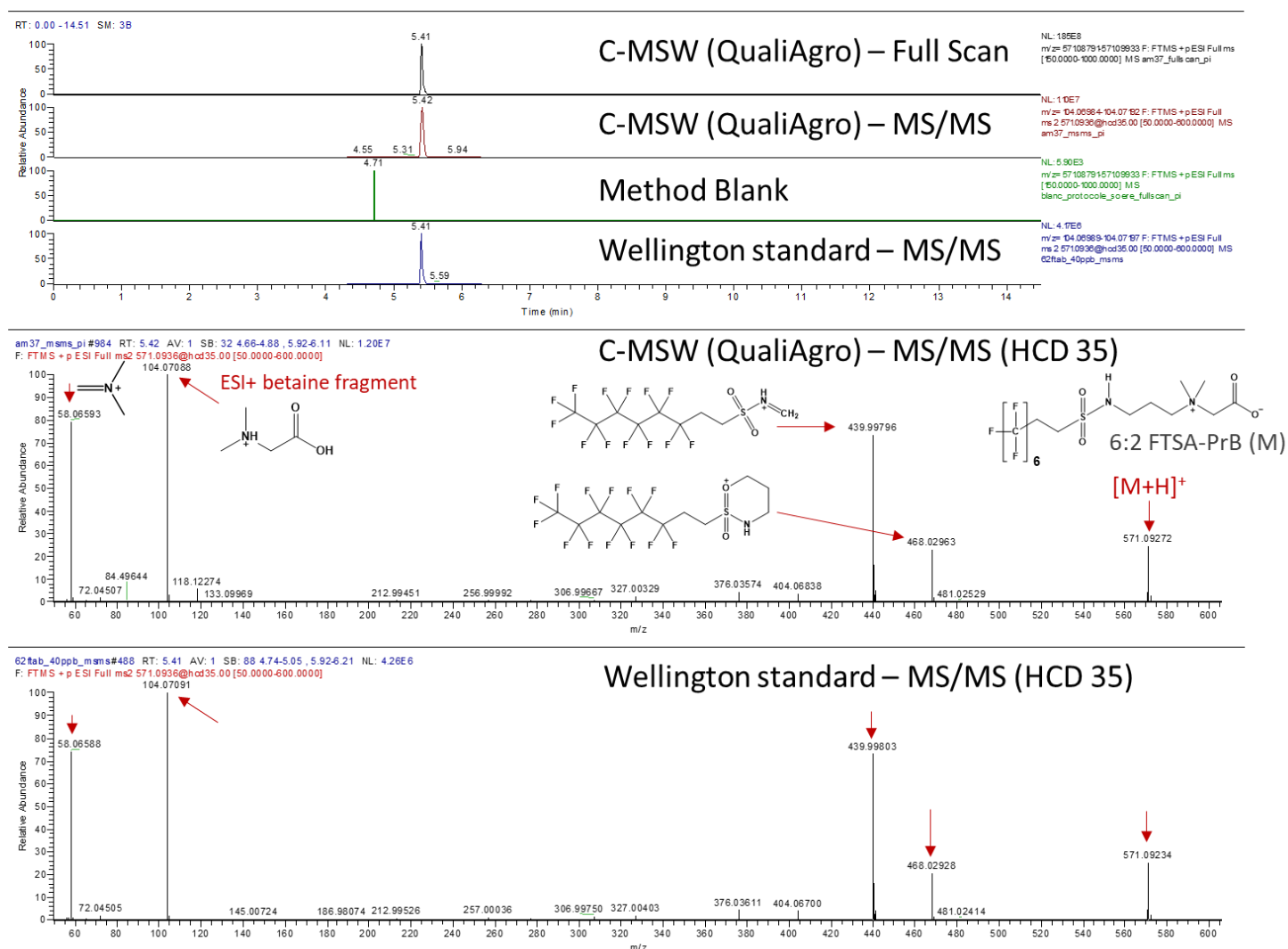

\begin{tabular}{|c|c|c|c|}
\hline & Theoretical $m / z$ & Observed $m / z$ & $\delta(\mathrm{ppm})$ \\
\hline Pseudomolecular ion $(\mathrm{M}+\mathrm{H})^{+}$ & 571.09362 & 571.09272 & $-1,6$ \\
\hline Fragment 1 & 104.07115 & 104.07091 & $-2,3$ \\
\hline Fragment 2 & 58.06567 & 58.06593 & 4,5 \\
\hline Fragment 3 & 439.99899 & 439.99796 & $-2,3$ \\
\hline Fragment 4 & 468.03029 & 468.02963 & $-1,4$ \\
\hline
\end{tabular}


(S2-t). 8:2 FTSA-PrB (8:2 FTAB), compost of residual municipal solid wastes (C-MSW) from QualiAgro site. An aqueous film-forming foam (Arctic Foam AFFF) was also analyzed for comparison.

\section{8:2 FTSA-PrB (8:2 FTAB)}

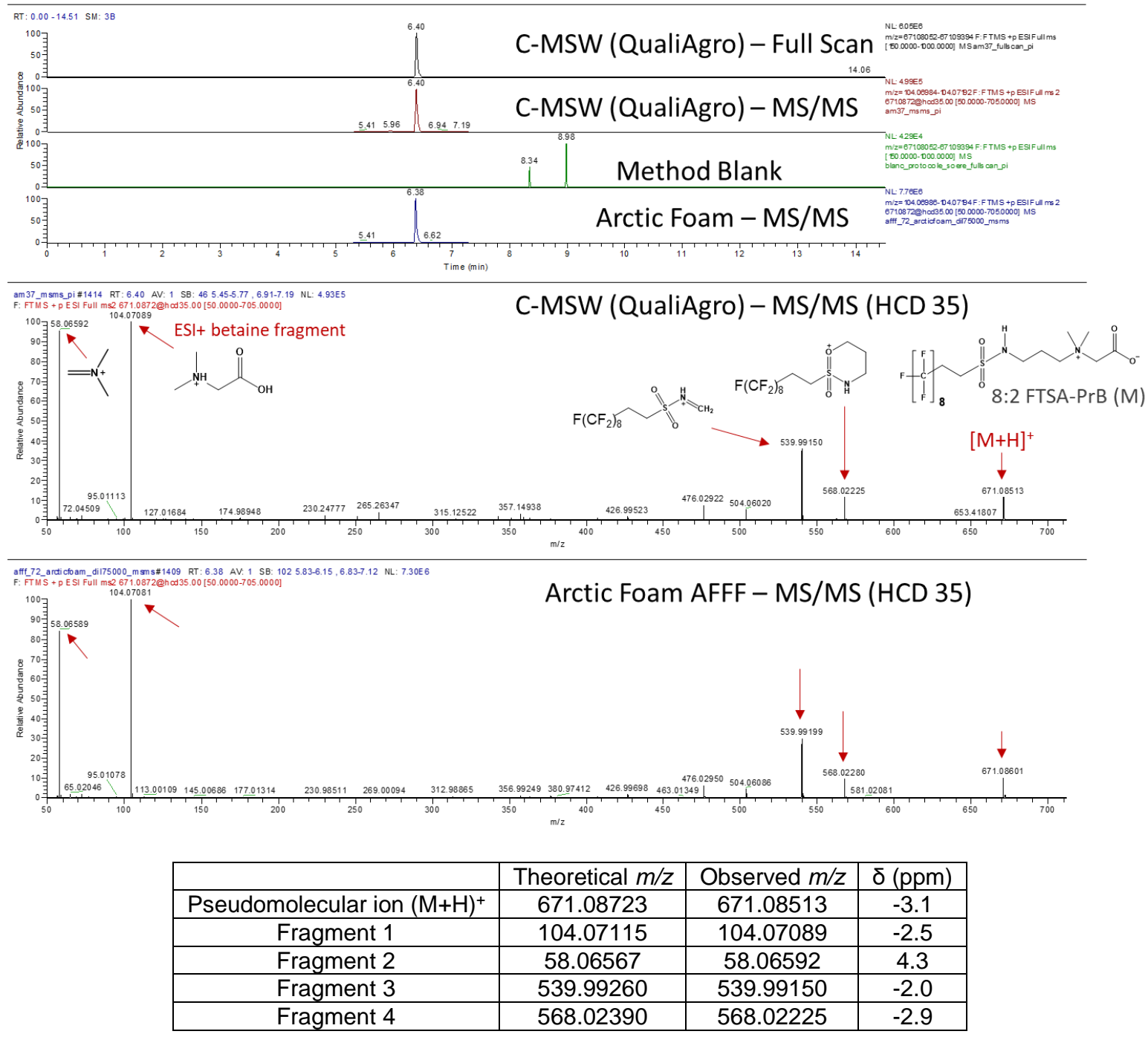


Figure S3. Principal component analysis biplot of active variables (seven PFAS superclasses) and individuals (OWP samples), performed on the OWP dataset (PCA type: Spearman). Individuals are color-coded according to the type of OWP (SLU: sewage sludge; C-GWS: compost of green wastes and sludge; C-BIOW: compost of biowastes; C-MSW: compost of municipal solid waste; DIG-UW: digestate of urban wastes; FYM-DC: farmyard manure of dairy cattle; PS: pig slurry; PM: poultry manure; C-FYM-DC: compost of farmyard manure of dairy cattle; DIG-PS: digestate of pig slurry; PSLU: paper sludge (mill sludge); ASH: combustion ashes).

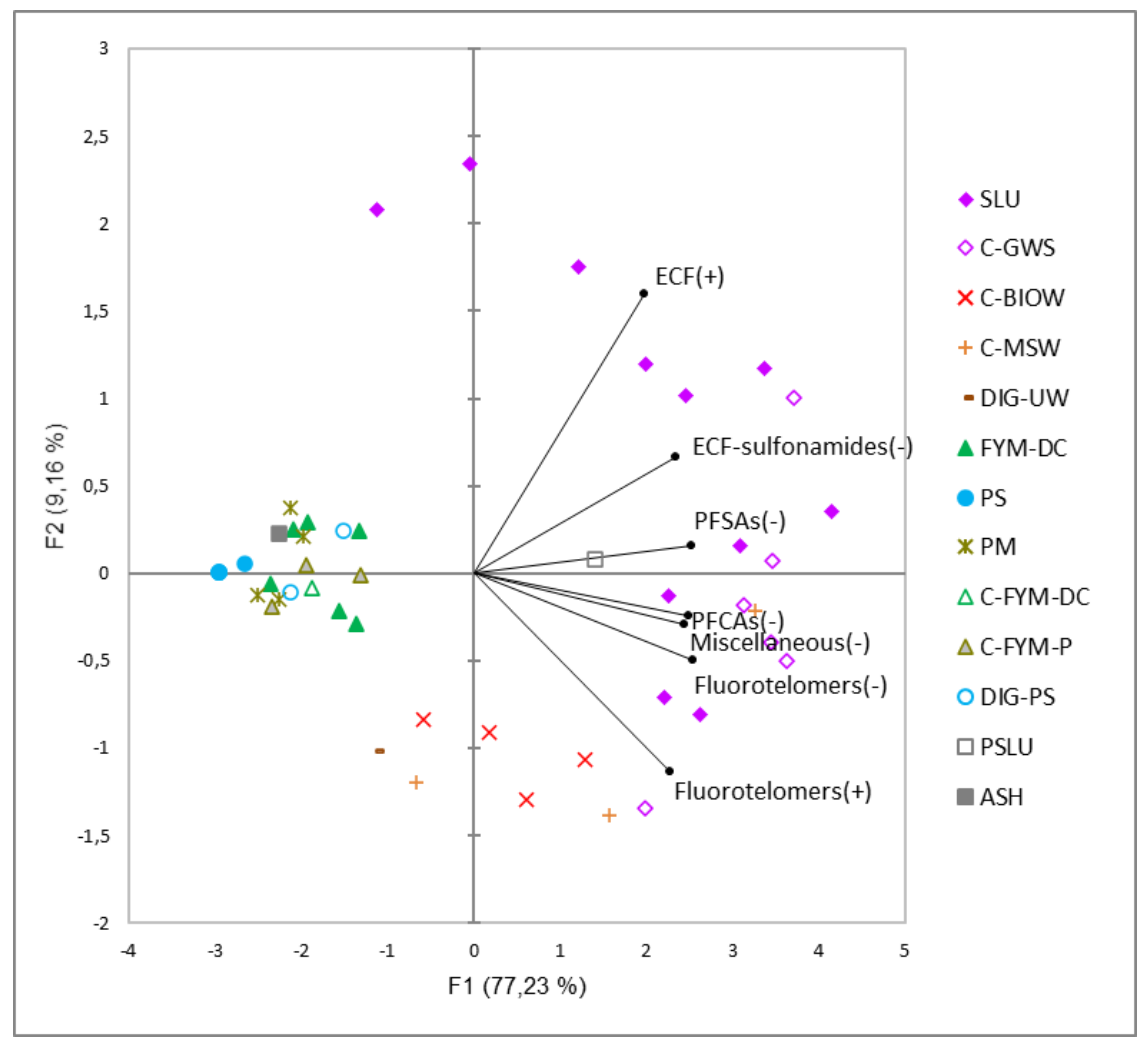


Figure S4. Summed PFAS in a sample of composted residual municipal solid waste (C-MSW, QA site; Year: 2013), according to different monitoring scenarios. The left panel shows the graphs for scenarios 1,2 and the right panel for all three scenarios. (Scenario 1) summed target PFAAs (PFCAs + PFSAs); (Scenario 2) summed target PFAAs and commonly targeted ESI(-) precursors (FOSA, Me/EtFOSA, Me/EtFOSAA, X:2 FTSA); (Scenario 3) summed PFAS detected in the present study ( $\Sigma_{160}$ PFAS). The largest contribution was attributed to the fluorotelomer sulfonamidopropyl betaines (X:2 FTSA-PrB), especially the 6:2 homolog (6:2 FTSA-PrB also known as 6:2 FTAB).
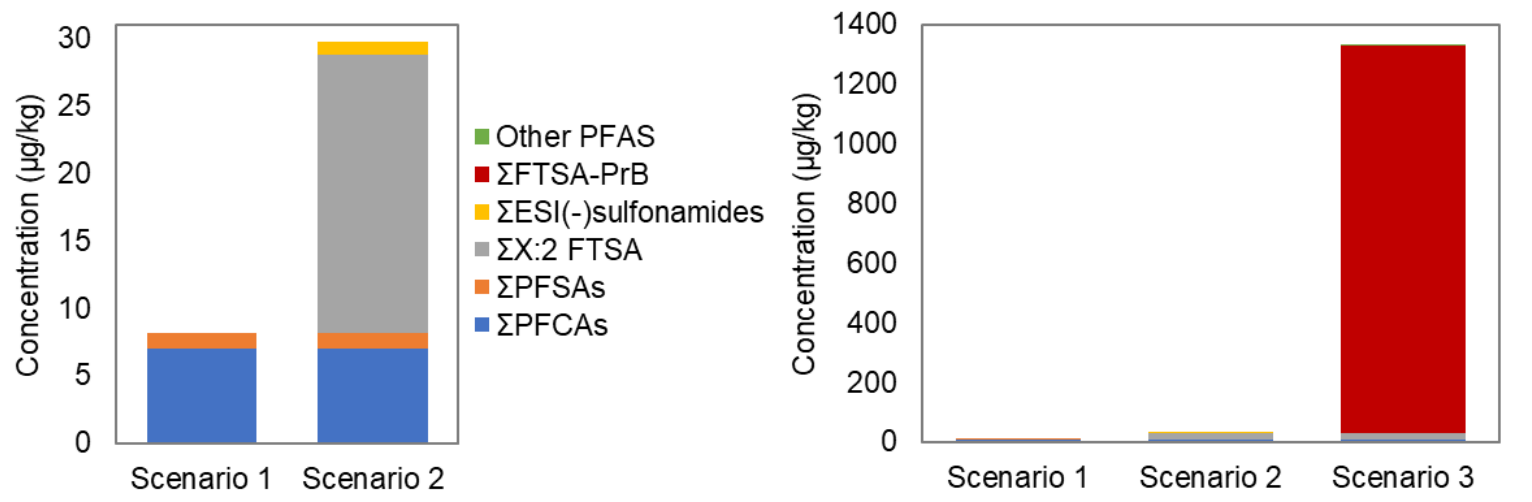


\section{References}

Choi, Y. J., Kim Lazcano, R., Yousefi, P., Trim, H., \& Lee, L. S. (2019). Perfluoroalkyl acid characterization in US municipal organic solid waste composts. Environmental Science \& Technology Letters, 6(6), 372-377.

Gallen, C., Drage, D., Kaserzon, S., Baduel, C., Gallen, M., Banks, A., Broomhall, S., \& Mueller, J. F. (2016). Occurrence and distribution of brominated flame retardants and perfluoroalkyl substances in Australian landfill leachate and biosolids. Journal of Hazardous Materials, 312, 55-64.

Munoz, G., Ray, P., Mejia-Avendaño, S., Duy, S. V., Do, D. T., Liu, J., \& Sauvé, S. (2018). Optimization of extraction methods for comprehensive profiling of perfluoroalkyl and polyfluoroalkyl substances in firefighting foam impacted soils. Analytica Chimica Acta, 1034, 74-84.

Paul, A. G., Jones, K. C., \& Sweetman, A. J. (2009). A first global production, emission, and environmental inventory for perfluorooctane sulfonate. Environmental Science \& Technology, 43(2), 386-392.

Sepulvado, J. G., Blaine, A. C., Hundal, L. S., \& Higgins, C. P. (2011). Occurrence and fate of perfluorochemicals in soil following the land application of municipal biosolids. Environmental Science \& Technology, 45(19), 8106-8112.

Suominen, K., Verta, M., \& Marttinen, S. (2014). Hazardous organic compounds in biogas plant end products-Soil burden and risk to food safety. Science of the Total Environment, 491, 192199.

Venkatesan, A. K., \& Halden, R. U. (2013). National inventory of perfluoroalkyl substances in archived US biosolids from the 2001 EPA National Sewage Survey. Journal of Hazardous Materials, 252, 413-418. 